\title{
Benchmark CFD Validation Data for Surface Combatant 5415 in PMM Maneuvers - Part I: Force/Moment/Motion Measurements
}

\author{
Hyunse Yoon ${ }^{1}$, Claus D. Simonsen ${ }^{2}$, Lanfranco Benedetti ${ }^{3}$, Joseph Longo ${ }^{1}$, Yasuyuki Toda ${ }^{4}$, \\ and Frederick Stern ${ }^{1^{*}}$ \\ ${ }^{1}$ IIHR - Hydroscience \& Engineering, the University of Iowa, Iowa City, IA 52242, U.S.A. \\ ${ }^{2}$ FORCE Technology, Lyngby, Denmark \\ ${ }^{3}$ Instituto Nazionale per Studi ed Esperienze di Architettura Navale, Rome, Italy \\ ${ }^{4}$ Department of Naval Architecture \& Ocean Engineering, Osaka University, Osaka, Japan
}

\begin{abstract}
Part I of this two-part paper presents benchmark CFD validation force/moment/motion measurements for surface combatant 5415 in planar-motion-mechanism (PMM) maneuvers. The experiments are conducted in the IIHR towing tank as part of an international collaboration for development/application of uncertainty analysis procedures and assessment of scale effects and facility biases. Stationarity and normality tests and statistical convergence errors, single and multiple run methods for obtaining the maneuvering mathematical model hydrodynamic derivatives, and the effects of heave, pitch and roll motions and fixed/free mount conditions are assessed. Assessment of the methods for obtaining the hydrodynamic derivatives is based on reconstructions of the measured force and moment time histories. Part 2 presents phase-averaged stereo PIV flow field measurements. The data is used as a test case at the SIMMAN 2008 and 2014 workshops on verification and validation of ship maneuvering simulation methods.
\end{abstract}

Keywords: Ship maneuvering; Mathematical modeling; Planar motion mechanism; Statistical convergence; Uncertainty analysis; Hydrodynamic derivative

\section{INTRODUCTION}

Traditionally maneuverability was a secondary consideration in comparison to resistance/propulsion and seakeeping in ship design; however, its identification as a major factor for

\footnotetext{
${ }^{*}$ Corresponding author: Frederick-stern@uiowa.edu; (319) 335-5215.
} 
navigational safety and the development of International Maritime Organization Standards for Ship Maneuverability (Resolution MSC.137(76), IMO 2002) has significantly increased its importance. Maneuvering simulation methods have been largely system-based approaches using maneuvering mathematical models and experimental data for estimating the hydrodynamics derivatives since surge, sway, yaw and roll are of primary importance and require viscous effects, whereas heave and pitch are of secondary importance and can be predicted using inviscid methods.

Recently, CFD methods have shown great promise for replacing experimental data for estimating the hydrodynamics derivatives and for free running simulations at both model and full scale. Significant progress has been made toward this goal by using Reynolds-averaged NavierStokes (RANS)-, unsteady RANS (URANS)-, or Detached Eddy Simulation (DES)-based codes for various hull forms in captive or free-running maneuvers. A few of the latest examples include steady-drift or -turning simulations by Tahara et al. (2002) for Series 60, Simonsen and Stern (2005) for Esso Osaka, Hyman et al. (2006) for Athena R/V, Bhushan et al. (2011) for naval combatant 5415, and Xing et al. (2012) for a tanker hull form. Dynamic captive-maneuvering simulations were done by Cura Hochbaum (2006) for a twin-screw ferry, Di Mascio et al. (2007) for a tanker, Simonsen et al. (2012) for a container ship, and Sakamoto et al. (2012a,b) for a naval combatant. Free-running simulations such as turning circle or zigzag maneuvers have been done by Bhushan et al. (2009) and Carrica et al. (2013) for a naval combatant and Dubbioso et al. (2012) for a twin-screw tanker. Free running CFD simulations with system identification have recently shown promise for the best and most efficient approach for estimating the hydrodynamic derivatives (Araki et al., 2012). Current CFD research focuses on maneuvering in waves (Sadat-Hosseini et al., 2014) and capsize (Hosseini et al., 2011). The $24^{\text {th }}-27^{\text {th }}$ International Towing Tank Conference (ITTC) Maneuvering Committee (MC) Reports (http://ittc.sname.org) provide detailed discussions/references for maneuvering physics, experiments and simulation methods.

The $24^{\text {th }} \mathrm{MC}$ has also raised the needs for new experimental benchmark data for maneuvering predictions, particularly in model scale, using modern hull forms. The committee recommended new benchmark hull forms including the present 5415 surface-combatant hull form, expecting well documented PMM and free-running model test data. More recently, the SIMMAN 2008 workshop on verification and validation of ship maneuvering simulation methods provided an overview of the state-of-the-art for the first time (Stern et al., 2011). The second workshop in 
2014 included shallow water test cases (http://www.simman2014.dk). Also noteworthy was the international collaboration on procurement of benchmark experimental data for captive and free running models used as test cases at the workshops.

Part I of this two-part paper presents benchmark CFD validation force/moment/motion measurements for surface combatant 5415 in planar-motion-mechanism (PMM) static drift and dynamic pure sway and yaw and yaw/drift maneuvers for several Froude numbers (Fr). The experiments are conducted in the IIHR towing tank as part of an international collaboration with FORCE Technology and INSEAN under the auspices of the $24^{\text {th }}-25^{\text {th }}$ ITTC MC for development/application of uncertainty analysis procedures and assessment of scale effects and facility biases. The results were adopted by the $25^{\text {th }}$ ITTC as Quality Manual Procedure 7.5-02-06.04 "Uncertainty Analysis: Forces and Moment Example for Planar Motion Mechanism Test." Stationarity and normality tests and statistical convergence errors, single and multiple run methods for obtaining the maneuvering mathematical model hydrodynamic derivatives, and the effects of heave, pitch and roll motions and fixed/free mount conditions are also assessed. Assessment of the methods for obtaining the hydrodynamic derivatives is based on reconstructions of the measured force and moment time histories. Part 2 presents phase-averaged stereo PIV flow field measurements. The data is used as a test case at the SIMMAN 2008 and 2014 workshops on verification and validation of ship maneuvering simulation methods.

\section{MATHEMATICAL MODELING AND PMM TEST}

\subsection{Mathematical modeling}

The present maneuvering mathematical modeling follows the Abkowitz (1964) approach. The modeling is for 3DOF surge, sway, and yaw motions in the horizontal plane. The approach is to describe the total hydrodynamic surge $X$ and sway $Y$ forces and yaw moment $N$ acting on a ship sailing on an unbounded calm water surface as general functions of the state variables at a certain instant such as surge, sway, and yaw velocities $(u, v, r$, respectively) and accelerations $(\dot{u}$, $\dot{v}, \dot{r}$, respectively) and rudder deflection angle $\delta$ such that $(X, Y, N)=f(u, v, r, \dot{u}, \dot{v}, \dot{r}, \delta)$. The modeling expands these general functions by using a $3^{\text {rd }}$-order multivariate Taylor series expansion based on the 'quasi-steady' assumption or equivalently the 'slow motion' assumption (Bishop and Parkinson, 1970). 
Abkowitz (1964) dropped a large number of the terms in the expansion equations with consideration of the symmetry in ship geometry between starboard and port sides. Further simplifications were made by assuming that there are no second- or higher order terms of the acceleration variables and no cross coupling between the acceleration and velocity variables. StrømTejsen and Chislett (1966) provides a full-set of the expansion equations with detailed derivations. In case of a bare hull without rudders and propellers, the expansion equations may be written as

$$
\begin{gathered}
X=X_{*}+X_{v v} v^{2}+X_{r r} r^{2}+X_{v r} v r+X_{u} \Delta u+X_{u u} \Delta u^{2}+X_{u u u} \Delta u^{3}+X_{v v u} v^{2} \Delta u \\
+X_{r r u} r^{2} \Delta u+X_{v r u} v r \Delta u+X_{\dot{u}} \dot{u} \\
\begin{array}{c}
Y=Y_{v} v+Y_{v v v} v^{3}+Y_{\dot{v}} \dot{v}+Y_{r} r+Y_{r r r} r^{3}+Y_{\dot{r}} \dot{r}+Y_{v r r} v r^{2}+Y_{r v v} r v^{2}+Y_{v u} v \Delta u \\
+Y_{v u u} v \Delta u^{2}+Y_{r u} r \Delta u+Y_{r u u} r \Delta u^{2} \\
\\
N=N_{v} v+N_{v v v} v^{3}+N_{\dot{v}} \dot{v}+N_{r} r+N_{r r r} r^{3}+N_{\dot{r}} \dot{r}+N_{v r r} v r^{2}+N_{r v v} r v^{2}+N_{v u} v \Delta u \\
+N_{v u u} v \Delta u^{2}+N_{r u} r \Delta u+N_{r u u} r \Delta u^{2}
\end{array}
\end{gathered}
$$

where $\Delta u=u-U_{0}$ and $U_{0}$ is the ship advance speed at the reference state, i.e., the steady straight-ahead running condition. The simplified derivatives expressions in equations (1)-(3), e.g., $Y_{v}=\partial Y / \partial v, Y_{v r}=\partial^{2} Y / \partial v \partial r$ and so on, are also known as the hydrodynamic derivatives.

Although this 3DOF modeling in equations (1)-(3) assumes no motions in the vertical plane, the real ship motions in the vertical plane are coupled with the motions in the horizontal plane and these vertical plane motions may influence considerably the forces and moment components in the horizontal plane, as pointed out by Chislett (1990). Thus, it is standard procedure in experimental maneuvering studies to allow ship model free motions in heave and pitch even for a captive model test, e.g., the ITTC Recommended Procedures and Guidelines 07-02-06-02 'Captive Model Test Procedures.'

A similar Taylor series expansion approach per Abkowitz (1964) is applicable to the equations of the heave $z$, pitch $\theta$, and roll $\phi$ motions in ship maneuvering for mathematical modeling. In this case Taylor series expansions involve an even larger number of the state variables as $(Z, M, K)=f(z, \theta, \phi, \dot{z}, \dot{\theta}, \dot{\phi}, \ddot{z}, \ddot{\theta}, \ddot{\phi}, u, v, r, \dot{u}, \dot{v}, \dot{r}, \delta)$, where $Z$ is heave force and $M$ and $K$ are pitch and roll moments, respectively. The number of terms in the expansions is quite large even after dropping many terms by using the same geometric considerations as for the previous 
$(X, Y, N)$ expansions. Further simplifications can be made by assuming $\Delta u=0$ and no crosscoupled terms between the vertical and horizontal plane motions in the expansion, e.g., $Z_{v v z} v^{2} z=0, Z_{r r \theta} r^{2} \theta=0$, etc. When the resulting expansion is combined with the rigid body motion equations (e.g., Fossen, 1994, pp. 30 or Lewandowski, 2004, pp. 12), the following $2^{\text {nd }}$ order differential equations can be written as

$$
\begin{aligned}
\left(m-Z_{\ddot{z}}\right) \ddot{z}- & Z_{\dot{z}} \dot{z}-Z_{z} z+\left(m x_{\mathrm{G}}-Z_{\ddot{\theta}}\right) \ddot{\theta}-\left(m U_{0}+Z_{\dot{\theta}}\right) \dot{\theta}-Z_{\theta} \theta-\left(m v+m x_{\mathrm{G}} r\right) \dot{\phi} \\
= & Z_{*}+Z_{v v} v^{2}+Z_{r r} r^{2}+Z_{v r} v r \\
\left(I_{y}-M_{\ddot{\theta}}\right) \ddot{\theta}+ & \left(m x_{\mathrm{G}} U_{0}-M_{\dot{\theta}}\right) \dot{\theta}-M_{\theta} \theta-\left(m x_{G}+M_{\ddot{z}}\right) \ddot{z}-M_{\dot{z}} \dot{z}-M_{z} z \\
& -\left(\left(I_{x}-I_{z}\right) r-m x_{\mathrm{G}} v\right) \dot{\phi}=M_{*}+M_{v v} v^{2}+M_{r r} r^{2}+\left(M_{v r}+m z_{\mathrm{G}}\right) v r \\
\left(I_{x}-K_{\ddot{\phi}}\right) \ddot{\phi}- & K_{\dot{\phi}} \dot{\phi}-K_{\phi} \phi \\
& =\left(K_{\dot{v}}-m z_{\mathrm{G}}\right) \dot{v}+K_{v} v+K_{v v v} v^{3}+K_{\dot{r}} \dot{r}+\left(m z_{\mathrm{G}} U_{0}+K_{r}\right) r+K_{r r r} r^{3} \\
& +K_{v r r} v r^{2}+K_{r v v} r v^{2}
\end{aligned}
$$

where, $m$ is the mass of the ship, $\left(I_{x}, I_{y}, I_{z}\right)$ the mass moment of inertia, and $\left(x_{\mathrm{G}}, y_{\mathrm{G}}, z_{\mathrm{G}}\right)$ the center of gravity (COG). Equations (4)-(6) use further simplifications $\left(y_{G}=0, I_{y}=I_{z}, \delta=0\right)$ and are linearized with respect to the vertical motion variables $z, \theta, \phi, \dot{z}, \dot{\theta}, \dot{\phi}, \ddot{z}, \ddot{\theta}$, and $\ddot{\phi}$.

\subsection{PMM test and hydrodynamic derivatives}

The evaluation of the hydrodynamic derivatives in the mathematical model equations (1)(3) is by using a planar motion mechanism (PMM) as first described by Gertler (1959) and Goodman (1960). PMM imposes pre-defined simple static or dynamic manoeuvers to a scale model ship and measures the resulting hydrodynamic forces and moments.

A static drift test is used to determine the static derivatives $\left(X_{*}, X_{v v}, Y_{v}, Y_{v v v}, N_{v}, N_{v v v}\right)$ by imposing steady $v$ such that the equations (1)-(3) are simplified as

$$
\left(\begin{array}{c}
X \\
Y \\
N
\end{array}\right)=\left(\begin{array}{c}
X_{*}+X_{v v} v^{2} \\
Y_{v} v+Y_{v v v} v^{3} \\
N_{v} v+N_{v v v} v^{3}
\end{array}\right)
$$

The tests are repeated over a range of various $v$ values to construct polynomial functions that best fit to the series of $(X, Y, N)$ data points. For example, a quadratic function $X(v)=A+B v^{2}$ 
is curve-fitted to the $X$ data to determine $X_{*}=A$ and $X_{v v}=B$ based on the simplified $X$ model in equation (7), and so on similarly for $Y$ and $N$ data to determine the rest of the static derivatives.

Dynamic PMM includes pure sway, pure yaw, and yaw-and-drift tests. A pure sway test is mainly to determine the linear acceleration derivatives $\left(Y_{\dot{v}}, N_{\dot{v}}\right)$ by imposing dynamically varying $v$ and $\dot{v}$ (e.g., $v=-v_{\max } \cos \omega t$ and $\dot{v}=\dot{v}_{\max } \sin \omega t$ ). A pure yaw test is to determine the rotary $\left(X_{r r}, Y_{r}, Y_{r r r}, N_{r}, N_{r r r}\right)$ and angular acceleration $\left(Y_{\dot{r}}, N_{\dot{r}}\right)$ derivatives by imposing dynamically varying $r$ and $\dot{r}$ (e.g., $r=r_{\max } \sin \omega t$ and $\dot{r}=\dot{r}_{\text {max }} \cos \omega t$ ). A yaw-and-drift test is a combined pure yaw test with the static drift test to determine the cross-coupled derivatives $\left(X_{v r}\right.$, $\left.Y_{v r r}, Y_{r v v}, N_{v r r}, N_{r v v}\right)$. Accordingly, equations (1)-(3) are reduced to simpler forms that can be rewritten in a harmonic form as

$$
\left(\begin{array}{c}
X \\
Y \\
N
\end{array}\right)=\left(\begin{array}{c}
X_{0} \\
Y_{0} \\
N_{0}
\end{array}\right)+\sum_{n=1}^{3}\left[\left(\begin{array}{c}
X_{\mathrm{C} n} \\
Y_{\mathrm{C} n} \\
N_{\mathrm{C} n}
\end{array}\right) \cos n \omega t+\left(\begin{array}{c}
X_{\mathrm{S} n} \\
Y_{\mathrm{S} n} \\
N_{\mathrm{S} n}
\end{array}\right) \sin n \omega t\right]
$$

where, the static component $\left(X_{0}, Y_{0}, N_{0}\right)$ and the cosine $\left(X_{\mathrm{C} n}, Y_{\mathrm{C} n}, N_{\mathrm{C} n}\right)$ and sine $\left(X_{\mathrm{S} n}, Y_{\mathrm{S} n}, N_{\mathrm{S} n}\right)$ harmonics are summarized in Table 1 for each dynamic test. Two different approaches are possible to evaluate hydrodynamic derivatives from these dynamic maneuvers, referred to as, the 'Multiple-Run (MR)' and the 'Single-Run (SR)' methods as introduced below.

1) MR method: This method uses curve-fittings of the mathematical models for the harmonics in equation (8) similarly with the evaluation method for the static derivatives. The test is repeated over a range of test parameters of interest (e.g., $r_{\max }$ or $\dot{r}_{\max }$ for pure yaw test) to construct polynomial functions that best fit to the series of the $(X, Y, N)$ harmonics data points. For example, a cubic function $Y_{\mathrm{S} 1}\left(r_{\max }\right)=C r_{\max }+D r_{\max }^{3}$ is curve-fitted to the $1^{\text {st }}$-order harmonic amplitude data of $Y$ time-histories obtained from the repeated pure yaw tests to determine $Y_{r}=C$ and $Y_{r r r}=4 D / 3$ by referring to the $Y_{\mathrm{S} 1}$ model for pure yaw in Table 1 . While all derivatives are available from the lowest non-zero harmonics (the $0^{\text {th }}$ - or the $1^{\text {st }}$-order), a few derivatives are also available from the higher order harmonics (the $2^{\text {nd }}$ - or the $3^{\text {rd }}$-order), e.g., $Y_{r r r}$ from $Y_{\mathrm{C} 3}$ or $N_{r r r}$ from $N_{\mathrm{C} 3}$. The former case is named as the $\mathrm{MR}_{\mathrm{L}}$ method and the latter as the $\mathrm{MR}_{\mathrm{H}}$ method. Table 2 provides a summary of the curve-fitting functions for both methods. 
2) SR method: This method directly solves the non-zero harmonics models in Table 1 to evaluate the derivatives, instead of using a curve fitting. For example, the rotary $Y$ derivatives are determined by solving the harmonics equations $Y_{\mathrm{S} 1}=Y_{r} r_{\max }+3 Y_{r r r} r_{\max }^{3} / 4$ and $Y_{\mathrm{S} 3}=$ $-Y_{r r r} r_{\max }^{3} / 4$ for $Y_{r}$ and $Y_{r r r}$ such that $Y_{r}=\left(Y_{\mathrm{S} 1}+3 Y_{\mathrm{S} 3}\right) / r_{\max }$ and $Y_{r r r}=-4 Y_{\mathrm{S} 3} / r_{\max }$. The experimentally obtained $Y_{\mathrm{S} 1}$ and $Y_{\mathrm{S} 3}$ values from a single-run of pure yaw test for a certain $r_{\max }$ condition are used to evaluate the solutions for $Y_{r}$ and $Y_{r r r}$. Thus, this method requires only a single realization of each dynamic test so that the method can be used for situations where a multiple number of realizations of the test are cost-prohibitive such as in CFD simulations. Table 3 provides the algebraic solutions for all the derivatives. For yaw-and-drift test, while all the crosscoupled derivatives can be evaluated by using the $0^{\text {th }}$ - or the $1^{\text {st }}$-order harmonics, $Y_{v r r}$ and $N_{v r r}$ derivatives are as well evaluated by using the $2^{\text {nd }}$-order harmonics. The former case is named as the $\mathrm{SR}_{\mathrm{L}}$ method and the latter case as the $\mathrm{SR}_{\mathrm{H}}$ method.

The heave, pitch, and roll motions during static or dynamic PMM maneuvers are predictable by solving the motion equations (4)-(6) for given maneuvers. The general solution of the motion equations may be written as $\xi=\xi_{\mathrm{h}}+\xi_{\mathrm{p}}$, where $\xi=(z, \theta, \phi)^{\mathrm{T}}$ and $\xi_{\mathrm{h}}$ is the homogeneous solution and $\xi_{\mathrm{p}}$ the particular solution. The homogeneous solution corresponds to the motions with the natural frequencies. The functional form of the particular solution of the coupled $z$ and $\theta$ equations (4) and (5) for a static drift maneuver of drift angle $\beta$ is

$$
\left(\begin{array}{c}
Z_{\mathrm{p}} \\
\theta_{\mathrm{p}}
\end{array}\right)=\mathbf{A}+\mathbf{B} \beta^{2}
$$

when $\phi=0$. The $\phi$ equation (6) is decoupled from equations (4) and (5), of which the particular solution for static drift maneuver is a cubic function as

$$
\phi_{\mathrm{p}}=A \beta+B \beta^{3}
$$

The particular solutions of $z, \theta$, and $\phi$ for the dynamic maneuvers in Table 1 are written as

$$
\left(\begin{array}{l}
z_{\mathrm{p}} \\
\theta_{\mathrm{p}} \\
\phi_{\mathrm{p}}
\end{array}\right)=\left(\begin{array}{l}
z_{0} \\
\theta_{0} \\
\phi_{0}
\end{array}\right)+\sum_{n=1}^{3}\left[\left(\begin{array}{c}
z_{\mathrm{C} n} \\
\theta_{\mathrm{C} n} \\
\phi_{\mathrm{C} n}
\end{array}\right) \cos n \omega t+\left(\begin{array}{c}
z_{\mathrm{S} n} \\
\theta_{\mathrm{S} n} \\
\phi_{\mathrm{S} n}
\end{array}\right) \sin n \omega t\right]
$$

where, the functional forms of the harmonics $\left(z_{0}, \theta_{0}, \phi_{0}\right)$ and $\left(z_{\mathrm{C} n}, \theta_{\mathrm{C} n}, \phi_{\mathrm{C} n}\right)$ and $\left(z_{\mathrm{S} n}, \theta_{\mathrm{S} n}\right.$, $\left.\phi_{\mathrm{Sn}}\right)$ are summarized in Table 4. 


\section{EXPERIMENTAL METHODS}

\subsection{Towing tank, PMM, and coordinate systems}

Tests are conducted at the IIHR towing tank facility shown in Fig. 1. The tank is $100 \mathrm{~m}$ long and $3.048 \mathrm{~m}$ wide and deep. The drive carriage is equipped with signal conditioning and data acquisition systems and pulls the PMM carriage. The PMM carriage supports the main PMM mechanical system and is also used as a point of attachment for model 5512.

Design and construction of the PMM is a collaborative effort by Sanshin Seisakusho Ltd. and Mori Engineering Ltd. for the mechanical and electrical systems, respectively. The mechanical system is a Scotch-yoke type which converts rotational motion of an $11 \mathrm{~kW}$ AC Servo motor to linear sway motion and rotary yaw motion. The Scotch-yoke is driven up to $0.25 \mathrm{~Hz}$ with maximum sway and yaw amplitudes of $500 \mathrm{~mm}$ and $30^{\circ}$, respectively. Linear and rotary potentiometers are installed to monitor and report the sway- and yaw-positions. The PMM is also presettable at drift angle $\beta$ between $\pm 30^{\circ}$, respectively.

Two right-handed Cartesian $x_{\mathrm{PMM}} y_{\mathrm{PMM}^{-}}$and $x y z$-coordinate systems are used. The inertial $x_{\mathrm{PMM}} y_{\mathrm{PMM}}$-coordinate system is fixed to the PMM with the $x_{\mathrm{PMM}}$ axis along the towing tank centerline to upstream and the $y_{\mathrm{PMM}}$ axis transversely to starboard. The origin of the $x_{\mathrm{PMM}_{\mathrm{M}}} y_{\mathrm{PMM}^{-}}$ coordinate system is located at the intersection of the tank centerline and a line that passes through the mid-ship point of the model. The non-inertial $x y z$-coordinate system is fixed to the moving model with $x$ along the model centerline, $y$ to starboard, $z$ downward, and the origin at the intersection of the mid-ship plane, center plane, and calm-water plane. PMM motions can be described by five quantities: 1) carriage speed $U_{\mathrm{C}}, 2$ ) sway motion amplitude $y_{\max }, 3$ ) yaw motion amplitude $\left.\psi_{\max }, 4\right)$ drift angle $\beta$, and 5) PMM frequency $f$. The following relations are used to setup static and dynamic tests:

$$
\begin{aligned}
& y_{\mathrm{PMM}}=-y_{\max } \sin \omega t \\
& \psi=-\psi_{\max } \cos \omega t+\beta
\end{aligned}
$$

where $\omega=2 \pi f$ and the heading angle $\psi$ is the angle between the model centerline and the tank centerline. The vector transformation between the ship-fixed and the PMM-fixed coordinate systems are given as follows:

$$
u=U_{\mathrm{C}} \cos \psi+v_{\mathrm{PMM}} \sin \psi
$$




$$
\begin{aligned}
\dot{u} & =\dot{v}_{\mathrm{PMM}} \sin \psi+r\left(v_{\mathrm{PMM}} \cos \psi-U_{\mathrm{C}} \sin \psi\right) \\
v & =v_{\mathrm{PMM}} \cos \psi-U_{\mathrm{C}} \sin \psi \\
\dot{v} & =\dot{v}_{\mathrm{PMM}} \cos \psi-r\left(U_{\mathrm{C}} \cos \psi+v_{\mathrm{PMM}} \sin \psi\right) \\
r & =r_{\mathrm{PMM}} \\
\dot{r} & =\dot{r}_{\mathrm{PMM}}
\end{aligned}
$$

where $v_{\mathrm{PMM}}=\dot{y}_{\mathrm{PMM}}, \dot{v}_{\mathrm{PMM}}=\ddot{y}_{\mathrm{PMM}}, r_{\mathrm{PMM}}=\dot{\psi}$, and $\dot{r}_{\mathrm{PMM}}=\ddot{\psi}$.

\subsection{Model ballasting and mounting}

The model geometry is DTMB model 5512, a 1:46.6 scale, $3.048 \mathrm{~m}$ long, fiber-reinforced Plexiglas hull, manufactured by the Naval Surface Warfare Center (NSWC). Model 5512 is a geosim of DTMB model 5415, a 1:24.8 scale $5.72 \mathrm{~m}$ model. The model is un-appended except for bilge keels, i.e., not equipped with shafts, struts, propellers, or rudders. To initiate transition to turbulent flow, a row of cylindrical studs are fixed at $0.05 \mathrm{~L}$ downstream from the FP (forward perpendicular), where $L$ is the model length between perpendiculars $\left(L_{P P}\right)$. The studs dimensions and placement on the model are in accordance with the recommendations by the $23^{\text {rd }}$ ITTC.

Table 5 summarizes model- and full-scale particulars and the mass properties. $x_{\mathrm{G}}$ and $y_{\mathrm{G}}$ are provided by NSWC. $z_{\mathrm{G}}$ is determined by using the added-ballast method as described by Irvine et al. (2008). $I_{x}$ and $I_{y}$ are determined by using the pendulum method, also as per Irvine et al. (2008) by measuring the roll and pitch gyradius, respectively. $I_{z}$ is determined by using a 'forced-yaw' method. The model is suspended in air from the 6DOF loadcell and PMM carriage and forced to follow a sinusoidal yawing motion, e.g., $\psi=\psi_{0} \sin \omega t$, with a prescribed yawing amplitude $\psi_{0}$ and cyclic frequency $\omega$. Then, $I_{z}$ is determined by measuring the resulting yaw moment $M$ and using the relationship $M_{0}=I_{z} \cdot \psi_{0} \omega^{2}$, where $M_{0}$ is the amplitude of the $M$ oscillation. A set of different combinations of $\psi_{0}$ and $\omega$ are tested and a least-square-error method is used to determine $I_{z}$.

Model ballasting and mounting is by using either a fixed- or free-mount, from which four different mounting conditions are devised: 1) Fixed at even keel condition $\left.\left(\mathrm{FX}_{0}\right), 2\right)$ Fixed at dynamic sinkage and trim condition $\left.\left(\mathrm{FX}_{\sigma \tau}\right), 3\right)$ Free to heave and pitch condition $\left(\mathrm{FR}_{\mathrm{z} \theta}\right)$, and 4$)$ Free to heave, pitch and roll condition $\left(\mathrm{FR}_{\mathrm{z} \theta \phi}\right)$. The $\mathrm{FX}_{0}$ and $\mathrm{FX}_{\sigma \tau}$ conditions are by using the fixedmount as shown in Fig. 2(a). The fixed-mount constrains the model in all motions. The model is first assembled rigidly to the fixed-mount. Then, ballasting is to the static waterline position for 
the $\mathrm{FX}_{0}$ condition and to a sinkage $\sigma=1.92 \times 10^{-3} \mathrm{~L}$ and a trim $\tau=-0.136^{\circ}$ (positive bow up) for the $\mathrm{FX}_{\sigma \tau}$ condition. The sinkage and trim values correspond to the dynamic sinkage and trim of the model in a straight-ahead towing at $F r=0.280$. The $\mathrm{FR}_{\mathrm{z} \theta}$ and $\mathrm{FR}_{\mathrm{z} \theta \phi}$ conditions are by using the free-mount as shown in Fig. 2(b). The model is first ballasted to the static waterline for both $\mathrm{FR}_{\mathrm{z} \theta}$ and $\mathrm{FR}_{\mathrm{z} \theta \phi}$. Then, the connection to the free-mount for the $\mathrm{FR}_{\mathrm{z} \theta}$ condition is by using rollerjoints that transmits the model heave and pitch motions but constrains the roll motion. The connection for the $\mathrm{FR}_{\mathrm{z} \theta \phi}$ condition is by using spherical joints that transmits all heave, pitch, and roll motions. The center of pitch motion is at the model mid-ship plane and the calm water line, and the center of roll motion is at the model center plane and the calm water line.

\subsection{Measurement variables and data reduction equations}

Measurement variables include carriage speed $U_{C}$, forces and moment $\left(F_{x}, F_{y}, M_{z}\right)$, and motions $\left(z_{\mathrm{mm}}, \phi, \theta\right)$. All variables are acquired as time histories through each carriage run. The motion variables $z_{\mathrm{mm}}, \phi$, and $\theta$ are measured only for free-model condition tests.

The data reduction equations (DRE's) for hydrodynamic forces and moment are:

$$
\begin{aligned}
& X=\frac{F_{x}+m\left(\dot{u}-v r-x_{\mathrm{G}} \dot{r}-y_{\mathrm{G}} \dot{r}\right)}{\frac{1}{2} \rho U^{2} L T} \\
& Y=\frac{F_{y}+m\left(\dot{v}+u r-y_{\mathrm{G}} r^{2}+x_{\mathrm{G}} \dot{r}\right)}{\frac{1}{2} \rho U^{2} L T} \\
& N=\frac{M_{z}+I_{z} \dot{r}+m\left[x_{\mathrm{G}}(\dot{v}+u r)-y_{\mathrm{G}}(\dot{u}-v r)\right]}{\frac{1}{2} \rho U^{2} L^{2} T}
\end{aligned}
$$

In equations (20)-(22), the surge, sway, and yaw velocity $(u, v, r)$ and acceleration $(\dot{u}, \dot{v}, \dot{r})$ are as per equations (14)-(19). $m$ and $I_{z}$ are the mass and yaw moment of inertia of the model. $x_{\mathrm{G}}$ and $y_{\mathrm{G}}$ are the center of gravity of the model. $\rho$ is the water density. $U$ is the ship speed defined in Section 3.1. $L$ and $T$ are the model length and draft, respectively. The above DRE's are applicable for all force and moment tests but reduce to equations (23)-(25) for the static drift tests

$$
\begin{aligned}
& X=\frac{F_{x}}{\frac{1}{2} \rho U_{\mathrm{C}}^{2} L T} \\
& Y=\frac{F_{y}}{\frac{1}{2} \rho U_{\mathrm{C}}^{2} L T}
\end{aligned}
$$




$$
N=\frac{M_{z}}{\frac{1}{2} \rho U_{C}^{2} L^{2} T}
$$

For the motion data, heave $z_{\mathrm{mm}}$ is non-dimensionalized with the model length $L$ whereas pitch $\theta$ and roll $\phi$ are used as measured,

$$
\begin{gathered}
z=z_{\mathrm{mm}} / L \\
\theta=\theta \\
\phi=\phi
\end{gathered}
$$

\subsection{Test conditions}

The variables in equations (14)-(19) are non-dimensionalized with ship length $L$ and speed $U=\sqrt{u^{2}+v^{2}}$ such that $u^{\prime}=u / U, \dot{u}^{\prime}=\dot{u} L / U^{2}, v^{\prime}=v / U, \dot{v}^{\prime}=\dot{v} L / U^{2}, r^{\prime}=r L / U$, and $\dot{r}^{\prime}$ $=\dot{r} L^{2} / U^{2}$, where $U \approx U_{\mathrm{C}}$ if $y_{\max } \omega / U_{\mathrm{C}} \ll 1$. The dynamic components of sway and yaw motions are constrained for static drift, i.e., $y_{\max }=0$ and $\psi_{\max }=0$ in equations (12) and (13), respectively, thus, $v^{\prime}=-\sin \beta$ from equation (16). The heading angle is maintained straight-ahead for pure sway, i.e., $\psi_{\max }=0$ and $\beta=0$ in equation (13) or $\psi=0$. Thus, $v^{\prime}=-v_{\max }^{\prime} \cos \omega t$ and $\dot{v}^{\prime}=\dot{v}_{\max }^{\prime} \sin \omega t$ from equations (16) and (17), respectively, where $v_{\max }^{\prime}=y_{\max } \omega / U_{\mathrm{C}}$ and $\dot{v}_{\max }^{\prime}$ $=y_{\max } \omega^{2} L / U_{\mathrm{C}}^{2}$. The model is always tangent to its path-line for pure yaw, i.e., $\psi_{\max }=$ $\tan ^{-1}\left(y_{\max } \omega / U_{\mathrm{C}}\right)$ in equation (13). Thus, $r^{\prime}=r_{\max }^{\prime} \sin \omega t$ and $\dot{r}^{\prime}=\dot{r}_{\max }^{\prime} \cos \omega t$ from equations (18) and (19), respectively, where $r_{\max }^{\prime}=\psi_{\max } \omega L / U_{\mathrm{C}}$ and $\dot{r}_{\max }^{\prime}=\psi_{\max } \omega^{2} L^{2} / U_{\mathrm{C}}^{2}$. In the remainder of the present study, the prime symbol is omitted from all of the aforementioned nondimensional variables for simplicity.

Table 6 summarizes the test conditions for IIHR facility. Static drift tests are for drift angle $\beta$ between $\pm 20^{\circ}$ corresponding to $v= \pm 0.342$. Pure sway test is for three maximum sway velocities $v_{\max }=0.035,0.070$, and 0.174 corresponding to the maximum drift angle $\beta_{\max }=$ $\tan ^{-1}\left(v_{\max }\right)=2^{\circ}, 4^{\circ}$, and $10^{\circ}$, respectively. Pure yaw test is for six maximum yaw rates, $r_{\max }$ up to 0.75 . Yaw-and-drift test is for three drift angles, $\beta=9^{\circ}, 10^{\circ}$, and $11^{\circ}$, with $r_{\text {max }}$ fixed at 0.3 . Tests are done at three $F r=0.138,0.280$, and 0.410 except for pure sway and yaw-and-drift tests which are done at one $F r=0.280$. For $F r=0.410$, the load-cell and PMM capacities limit the test conditions $\beta$ up to $12^{\circ}$ for static drift test and $r_{\max }$ up to 0.45 for pure yaw test. Tests are repeated 12 times for the cases marked in bold characters in Table 6 for the UA purposes discussed later in Section 5. The tests of $\mathrm{FX}_{0}$ and $\mathrm{FR}_{\mathrm{z} \theta}$ mount conditions cover the entire test cases listed 
above. The tests of $\mathrm{FX}_{\sigma \tau}$ mount condition covers only the static drift, pure sway, and pure yaw cases at one $F r=0.280$. The tests of $\mathrm{FR}_{\mathrm{z} \theta \phi}$ mount condition covers the entire static drift cases but only the UA cases of dynamic tests.

For dynamic tests, the PMM cyclic frequency $\omega=2 \pi f$ values are examined for possible unfavorable hydrodynamic effects such as memory effects or tank resonance. The examination follows the ITTC recommended procedures and guidelines 7.5-02-06-02 'Captive Model Test Procedures' for three non-dimensional frequencies $\omega_{1}^{\prime}=\omega L / U_{\mathrm{C}}, \omega_{2}^{\prime}=\omega \sqrt{L / g}$, and $\omega_{3}^{\prime}=$ $\omega U_{\mathrm{C}} / g$, where $g$ is the gravity. The restrictions of these non-dimensional frequency values are interpreted as avoiding non-stationary lift and memory effects for $\omega_{1}^{\prime}$, tank resonance for $\omega_{2}^{\prime}$, and unrealistic combinations of pulsation and translation for $\omega_{3}^{\prime}$. The empirical values provided by the ITTC guidelines are $\omega_{1}^{\prime}=1 \sim 4, \omega_{2}^{\prime}=0.15 \sim 0.2$ and $\omega_{3}^{\prime}<<0.25$. For the present study, $\omega_{1}^{\prime}=$ $1.14 \sim 3.13, \omega_{2}^{\prime}=0.27 \sim 0.88$, and $\omega_{3}^{\prime}=0.04 \sim 0.34$ are selected in considerations of the limitations by the facility dimension and the PMM capability.

Flow Reynolds number $R e=U_{\mathrm{C}} L / v$ is between $1.94 \times 10^{6} \sim 7.31 \times 10^{7}$, depending on the water temperature on the day of each test, where $v$ is the kinematic viscosity of fresh water. Typical water temperature was $13.5{ }^{\circ} \mathrm{C}, 11.5{ }^{\circ} \mathrm{C}, 21.5{ }^{\circ} \mathrm{C}$, and $23.0{ }^{\circ} \mathrm{C}$ for the $\mathrm{FX}_{0}, \mathrm{FX}_{\sigma \tau}, \mathrm{FR}_{z \theta}$, and $\mathrm{FR}_{\mathrm{z} \theta \phi}$ tests, respectively.

\subsection{Measurement systems}

Carriage speed is measured with a custom-made speed circuit. The operating principle is integer pulse counting at a wheel-mounted encoder. The hardware consists of an 8000-count optical encoder, carriage wheel, and sprocket pair and chain. Linear resolution is $0.15 \mathrm{~mm} / \mathrm{pulse}$. The speed circuit is periodically bench-calibrated to determine and adjust the frequency input/voltage output transfer function. The measurement details are presented in Longo and Stern (2005).

Forces and moment are measured with an Izumi six-component strain-gage type load cell and six-channel Izumi amplifiers for signal conditioning. Maximum force and moment ranges are $500 \mathrm{~N}$ for $F_{x}, F_{y}, F_{z}$ and $50 \mathrm{~N}-\mathrm{m}, 50 \mathrm{~N}-\mathrm{m}, 200 \mathrm{~N}-\mathrm{m}$ for $M_{x}, M_{y}, M_{z}$, respectively. The load cell is calibrated statically on a test stand using standard weights and internally at the amplifiers periodically. 
Motions are measured using a Krypton Electronic Engineering Rodym DMM motion tracker. The Rodym DMM is a camera-based measurement system that triangulates the position of a target in 3D space for contactless measurement and evaluation of 6DOF motions. The hardware consists of a camera module comprising three fixed CCD cameras, target with 1-256 lightemitting diodes (LED's), camera control unit, hand-held probe with six LED's, and PC. Krypton software is used for system calibration and data acquisition and reduction. More details of the measurement are presented in Irvine et al. (2008).

\subsection{Data acquisition and reduction procedures}

For the test setup, model 5512 is mounted on the tank centerline. The six-component loadcell is mounted between the PMM center-post and the fixed- or the free-mount as shown in Fig. 2(a) and (b), respectively. The Krypton camera module is mounted facing backward from the rear underside of the drive carriage to gain an unobstructed view of the LED target. The target is mounted near the bow and over the model centerline such that the LED's face the camera module. 6DOF motion data is reported from the camera controller at $40 \mathrm{~Hz}$.

To initiate data acquisition procedures, the PMM is activated and ten seconds elapse to allow enough time for the model motion to reach a steady rate. Then, the carriage is started then accelerates through $10 \mathrm{~m}$ to a constant speed. Data acquisition commences after traveling another $10 \mathrm{~m}$, which allows the unsteady free surface to develop and reach a state where it is not in transition. Data acquisition is by using an onboard 16-channel AD card and occurs at $100 \mathrm{~Hz} / \mathrm{channel}$ for 30, 20, 10 seconds for cases where $F r=0.138,0.280,0.410$, respectively.

For data reduction, static test variables are time-averaged. For dynamic tests, each varia-

ble time histories are reconstructed with a $6^{\text {th }}$-order Fourier series (FS) equation as per the following expression

$$
x(t)=x_{0}+\sum_{n=1}^{6}\left[x_{\mathrm{C} n} \cos (2 \pi n f t)+x_{\mathrm{S} n} \sin (2 \pi n f t)\right]
$$

where $x(t)$ can be any force, moment, or motion variable. In Eq. (29), $x_{0}$ is the static component and $x_{\mathrm{C} n}$ and $x_{\mathrm{S} n}$ are the $n^{\text {th }}$-order FS cosine and sine coefficients of $x(t)$, respectively. Alternatively, Eq. (29) can also be expressed as 


$$
x(t)=A_{0}+\sum_{n=1}^{6} A_{n} \cos \left(2 \pi n f t+\varphi_{n}\right)
$$

where $A_{0}=x_{0}$ and $A_{n}=\sqrt{x_{\mathrm{C} n}^{2}+x_{\mathrm{S} n}^{2}}$ and $\varphi_{n}=-\tan ^{-1}\left(x_{\mathrm{S} n} / x_{\mathrm{C} n}\right)$ are the amplitude and phase of the $n^{\text {th }}$-order harmonics component of $x(t)$, respectively. All periodic data is expressed and output through one PMM cycle. Finally, the hydrodynamic derivatives are computed as presented in Section 2 as the last data reduction step.

\section{STATISTICAL CONVERGENCE}

Data quality is assessed using statistical and spectral analysis following Bendat and Piersol (1966). Data are first tested for randomness, stationarity, and normality, and then statistical convergence is evaluated. Testing for randomness is by observing the data time history and inspecting the frequency spectrum of the data via Fast Fourier Transform (FFT) for an obviously non-stationary or non-random data. Stationarity test is by using the 'Reverse Arrangement Test,' a non-parametric statistical procedure also known as a 'Trend Test.' Data normality is examined by performing a Chi-square $\left(\mathrm{X}^{2}\right)$ goodness-of-fit test. Statistical convergence is done by monitoring the convergence of the confidence interval size of the mean value and evaluating a statistical convergence error. Example results are summarized in Table 7 for a static drift test at $\beta=10^{\circ}$ and a dynamic pure yaw test at $r_{\max }=0.3$ cases, both at $F r=0.280$ and for the $\mathrm{FR}_{\mathrm{z} \theta \phi}$ mount condition. Tests were done for 12 and 11 repeat runs, respectively, to emphasize the repeatability of the results.

\subsection{Time-histories and dominant frequencies}

Fig. 3 shows a set of static drift test variables time-histories (left column) and their FFT results (right column). FFT's are shown for all repeat runs together to emphasize the repeatability. Time-histories of $U_{C}, F_{x}, F_{y}, M_{z}$ data exhibit apparent random fluctuations with peak frequencies typically near $1.25,2.5,3,4,5,7,8$, and $10 \mathrm{~Hz}$. The frequency $1.25 \mathrm{~Hz}$ is from the carriage wheel frequency, $f_{\text {wheel }}=U_{\mathrm{C}} / \pi D$, where $D=381 \mathrm{~mm}$ (15 inch) is the wheel diameter, and 2.5 $\mathrm{Hz}$ is the $2^{\text {nd }}$-order harmonic of $f_{\text {wheel }}$. The other peak frequencies are determined from the natural frequencies (5 and $7 \mathrm{~Hz}$ ) and the mechanical vibrations $(3,4,7,8$, and $10 \mathrm{~Hz})$ of the loadcell, PMM, and towing carriage or some combinations of those, which were identified from indepen- 
dent measurements. $Z_{\mathrm{mm}}$ and $\theta$ are a superposition of a transient oscillation and random fluctuations. The transient oscillations are usually at a frequency of $f_{\operatorname{tr}} \approx 0.25 \mathrm{~Hz}$ due to a start-up transient that decays with time. $\phi$ is almost steady but seemingly exhibits a weakly transient oscillation at $0.13 \mathrm{~Hz} . z_{\mathrm{mm}}$ and $\phi$ also show the wheel frequency $f_{\text {wheel }}$.

Fig. 4 shows the dynamic test variables time-histories and their FFT's similarly as in Fig. 3. Contrary to the static test case, all variables time-histories exhibit complex-periodic oscillations plus random noise except for $U_{C}$ that is essentially the same as for the static drift test. The dominant frequency of the periodic oscillation is the excitation frequency (i.e., the PMM frequency) $f=0.134 \mathrm{~Hz}$ for $F_{y}, M_{z}, \phi$ and the $2^{\text {nd }}$-order harmonic $2 f=0.268 \mathrm{~Hz}$ for $F_{x}, z_{\mathrm{mm}}, \theta$. The peak frequencies of the random noise are similar as for static drift data but some frequencies such as $5,10 \mathrm{~Hz}$ are less distinctive from the FFT's of pure yaw $F_{x}, F_{y}, M_{z}$. Fig. 5 shows timehistories of the FS harmonics, $A_{n}(t)$, of the data time-histories shown in Fig. 4. The construction of $A_{n}(t)$ is initiated by taking a time-window of size corresponding to one period $P=1 / f$, i.e., for a time interval $(t-P, t)$, and then by using equation (30) as the time-window is 'marched' along the time axis. In Fig. 5, the harmonics values are presented in a normalized form, $A_{n}^{*}=$ $A_{n} / \overline{A_{n}}$, where $\overline{A_{n}}$ is the time-average of $A_{n}(t)$. The harmonics time-histories starts from a dimensionless time point $t / P=1$ as the FS procedure requires at least one complete period of data. If a variable has $A_{n}^{*}=1$ for all $n$, i.e., all its harmonics are invariant in time, then it is considered that the variable has reached a steady state. In general, lower-order harmonics such as $A_{0}, A_{1}, A_{2}$ exhibit a better steadiness with $A_{n}^{*} \approx 1$ than higher-order harmonics. The amount of harmonics change in time, $\delta A_{n}^{*}=\left(A_{n, \max }-A_{n, \min }\right) / \overline{A_{n}}$, is the smallest 0.01 for $A_{1}$ 's of $F_{y}, M_{z}, \phi$, moderate $0.1 \sim 0.5$ for $A_{0}$ and $A_{2}$ of $F_{x}, z_{\mathrm{mm}}, \theta$, and the largest $1 \sim 3$ for other higher-order harmonics of all variables.

\subsection{Stationarity and normality tests}

The time-histories are tested for stationarity to detect the presence of underlying trends or variations with time. The following assumptions are made for stationary test. 1) If the data of interest are stationary, the statistical properties computed for each sequence of short time intervals will not vary significantly from one time interval to the next. 2) Verification of weak stationarity (time invariance of the mean value and autocorrelation function) will be acceptable. 3) If the mean square value (or the variance) of the data of interest is stationary, then the autocorrelation 
function for the data is also stationary. Lastly, 4) the sample record of the data to be investigated is very long compared to the random fluctuations of the data in time.

The stationarity test is done by using a non-parametric statistical procedure known as the 'Reverse Arrangement Test.' Data records of any variable $x$ are divided into a sequence of number $N$ equal time intervals and the sequence of piecewise mean $(\bar{x})$ and variance $\left(\overline{x^{2}}\right)$ values is tested. For the sequence, for example, $\bar{x}_{i}, i=1,2,3, \ldots, N$, a reverse arrangement is defined when $\bar{x}_{i}>\bar{x}_{j}$ for $i<j$. Then, the total number of reverse arrangement is denoted by $A$. If $A$ is too small or too large (more specifically, out of an acceptance region $65 \leq A \leq 125$ for $N=20$ ), a hypothesis that the variations in the sequence of $\bar{x}$ and $\bar{x}^{2}$ sample values are random and display no trend is rejected at a $5 \%$ significance level. Note that a too-small $A$ value indicates a presence of an ascending trend, whereas a too-large $A$ value indicates presence of a descending trend.

Table 7 presents the reverse arrangement $A$ values obtained by testing the static variables time-histories shown in Fig. 3 and the dynamic variables harmonics time-histories shown in Fig. 4. For the latter case, only the $A_{n}$ values of the dominant harmonics $\left(A_{1}\right.$ for $F_{y}, M_{z}, \phi$ and $A_{2}$ for $\left.F_{x}, z_{\mathrm{mm}}, \theta\right)$ are presented. From the static variables, $U_{C}, M_{z}, \phi$ failed the test for $\bar{x}$ with $A=134$, 132, 150, respectively, indicating evidence of presence of a descending trend in the mean. From the dynamic variables, $F_{x}, \theta$ failed the test for $\bar{x}$ with $A=173,190$, respectively, and $F_{y}$, $\phi$ with $A=22,25$, respectively, revealing evidence of a descending trend in $A_{2}$ for the former variables and an ascending trend in $A_{1}$ for the later variables. However, all of the static and dynamic variables passed the test for $\overline{x^{2}}$, i.e., no evidence of trend in variables variance values with time. Consequently, for the given specific time-histories (shown in Fig. 3 and Fig. 4), the static $F_{x}, F_{y}$, $z_{\mathrm{mm}}, \theta$ and dynamic $M_{z}, z_{\mathrm{mm}}$ variables are considered as stationary. By examining all of the time-histories cases collected from a total of 12 static and 11 dynamic repeat tests, all the static variables except for $U_{C}$ and $\phi$ are accepted as stationary by a ratio of 2 times out of 3 , whereas all the dynamic variables only 1 time out 4, respectively. For dynamic variables, other nondominant harmonics components exhibit a similar behavior.

Data normality is examined by performing a Chi-square $\left(\mathrm{X}^{2}\right)$ goodness-of-fit test. The procedure is to form a frequency histogram of the data and use a Chi-square distribution as a measure of the discrepancy between the observed frequency and the theoretical expected frequency. For the test a class interval size $K=39$ was used following the Minimum Optimum 
Number of Class Intervals (Williams, 1950) for a sample size $N=2,000$. In this case, for a hypothesis of normality, the acceptance region is $\mathrm{X}^{2} \leq 51$ for a degree of freedom $n=K-3=36$ and at a 5\% level of significance. As presented in Table 7, the normality hypothesis is rejected for all static variables except for $F_{x}$ and $F_{y}$ and all dynamic variables for the specific test cases shown in Fig. 3 and Fig. 4. By observing the aforementioned repeat test cases, the static variables $F_{x}, F_{y}$, $M_{z}$ were accepted for the normality hypothesis generally by a ratio of 1 out of 2 times.

\subsection{Statistical convergence error}

For a stationary random variable $x$ of which true mean is $\mu_{x}$, a sample mean $\bar{x}$ can be considered as converged if $\left|\bar{x}-\mu_{x}\right| \leq \varepsilon$, where $\varepsilon$ can be any small positive number. However, usually $\mu_{x}$ is unknown for most practical applications. Thus, the convergence is often stated statistically with a probability $P=\operatorname{Prob}\left[\left|\bar{x}-\mu_{x}\right| \leq c \cdot s_{x} / \sqrt{N}\right]$, where $c$ is a constant, $s_{x}$ is the sample standard deviation of $x$, and $N$ is the sample size. For example, if the sample data follow a normal distribution, then $P=1-\alpha$ for $c=t_{n}$, where $t_{n}$ is the Student's $t$-statistic corresponding to $\alpha$. On the other hand, if the sample distribution type is unknown $P \geq 1-1 / c^{2}$ by using the Tchebycheff's inequality. Accordingly, a statistical convergence error may be defined as

$$
E_{S C}(\%)=\left(\frac{c \cdot s_{x}}{\sqrt{N}}\right) / \bar{x} \times 100
$$

For $P=0.95$, in other words, for a $95 \%$ confidence level, $c=2.0$ is used when the data follows a normal distribution, whereas $c=4.5$ is used when the type of data distribution is unknown.

Statistical convergence error $E_{\mathrm{SC}}(\%)$ values are presented in Table 7 for the specific static and dynamic test cases shown in Fig. 3 and Fig. 4, respectively. The static $F_{x}$ and $F_{y}$ follow a normal distribution as per the normality test and $E_{\mathrm{SC}}(\%)=1.21$ and 0.42 , respectively, by using $c=2$. Whereas $U_{C}, M_{z}, z_{\mathrm{mm}}, \theta$, and $\phi$ data fail the normality test thus $c=4.5$ is used. The convergence error is still satisfactorily small for all those variables with $E_{\mathrm{SC}}(\%)<1$. The harmonics data of dynamic variables are not stationary and $E_{\mathrm{SC}}(\%)$ may not be applicable in a strict sense. Nonetheless, recalling from the time-histories inspections in Section 4.1 that $\delta A_{n}^{*}$ values are very small for the $A_{1}$ 's of $F_{y}, M_{z}, \phi$ and moderate for the $A_{2}$ 's of $F_{x}, z_{\mathrm{mm}}, \theta$, thus those variables are possibly weakly non-stationary. Bearing this in mind, $E_{\mathrm{SC}}(\%)$ is fairly small $<1$ for all variables. These results were quite consistent through the repeat test runs. Consequently, it is concluded 
that most of the static variables are stationary and well converged and that the dynamic variables are possibly weakly non-stationary.

\section{UNCERTAINTY ANALYSIS (UA)}

The present UA procedure is developed in an international collaboration among three towing tank facilities (IIHR, FORCE, and INSEAN) and the $24^{\text {th }}-25^{\text {th }}$ ITTC MC, as previously mentioned in Section 1. The collaboration includes overlapping tests using the same model geometry and test matrix for comparisons of the results and for identification of possible facility biases and scale effects. The basis of the UA procedure was first developed by FORCE (Simonsen 2004) following the AIAA (1999), ANSI/ASME (1998), and AGARD (1994) standard and guidelines. Afterwards, the procedure was applied at INSEAN (Benedetti et al. 2006) and extended herein by including two conceptual biases, the asymmetry bias and the facility bias (or certification), as presented in the following sections. Initiation of the present collaboration occurred prior to release of a newer standard ASME PTC 19.1-2005 (ASME 2005), thus the newer standard was not used. Revisions of the present procedure compliant to the new standard are left as future work.

\subsection{UA methodology}

The approach includes errors/uncertainties definitions, systematic/random categorizations, and large sample size/normal distribution assumptions. The procedure is based on estimates of systematic bias $(B)$ and random precision $(P)$ limits, and their root-sum-square (RSS) combination to ascertain total uncertainty $(U)$. Bias limits are estimated with consideration of elemental error sources for individual variables, whereas precision limits are estimated end to end. A 95\% confidence level is achieved through careful estimations of the bias errors and usage of a multiple test approach for the precision errors.

Limitations of the present UA procedures are listed as follows: The effect of data conditioning such as filtering or fairing, for example, Fourier Series (FS) reconstructions for the measured forces /moment and motions is not counted in this UA procedure. This procedure also assumes that the model ship is free to heave and pitch, and fixed in roll. The effect of deviations from the upright position such as roll or heel angle is not considered in this procedure. Carriage speed is assumed constant, so the effect of acceleration caused by fluctuating carriage speed dur- 
ing runs is not considered. Lastly, this procedure does not provide UA for hydrodynamic derivatives derived from the forces and moment data or their effect on the full-scale maneuvering simulations.

\subsection{Bias limit}

For any result variable $r$ of which data reduction equation (DRE) is $r=r\left(x_{1}, x_{2}, \ldots, x_{J}\right)$, bias limit is determined with an error propagation equation

$$
B_{r}^{2}=\sum_{i=1}^{J} \theta_{x_{i}}^{2} B_{x_{i}}^{2}
$$

where $\theta_{x_{i}}=\partial r / \partial x_{i}$ are the sensitivity coefficients and $B_{x_{i}}$ are elemental bias in $x_{i}$. Sensitivity coefficients are evaluated analytically and elemental biases are assumed uncorrelated.

For the forces and moment variables $r=X, Y, N$, fourteen $\left(B_{L}, B_{T}, B_{x_{\mathrm{G}}}, B_{y_{\mathrm{G}}}, B_{m}, B_{I_{z}}, B_{\rho}\right.$, $\left.B_{u}, B_{v}, B_{r}, B_{\dot{u}}, B_{\dot{v}}, B_{\dot{r}}, B_{F}\right)$ and five $\left(B_{L}, B_{T}, B_{\rho}, B_{U_{\mathrm{C}}}, B_{F}\right)$ elemental biases are identified from the DRE's (20)-(22) for dynamic tests and (23)-(25) for static tests, respectively, which can be rewritten in a functional form, $r=r\left(L, T, x_{\mathrm{G}}, y_{\mathrm{G}}, m, I_{z}, \rho, u, v, r, \dot{u}, \dot{v}, \dot{r}, F\right)$ and $r=r(L, T, \rho$, $\left.U_{\mathrm{C}}, F\right)$, respectively, where $F=F_{x}, F_{y}$, or $M_{z}$. For dynamic tests, $B_{u}, B_{v}, B_{r}, B_{\dot{u}}, B_{\dot{v}}, B_{\dot{r}}$ are determined from elemental biases $B_{U_{\mathrm{C}}}, B_{y_{\max }}, B_{\psi_{\max }}, B_{\beta}, B_{\omega}, B_{t}$, propagated through their own DRE's (12)-(13) and then through equations (14)-(19).

The bias limits of the most fundamental error sources are summarized in Table $8 . B_{L}$ is from the model fabrication precision of $\pm 1 \mathrm{~mm}$ in all directions. $B_{T}$ is from the errors in model ballasting. $B_{x_{\mathrm{G}}}$ and $B_{y_{\mathrm{G}}}$ are associated with the deviation of the actual model COG from the designed position during the model ballasting and installation procedures. $B_{m}$ is from the accuracy of weight balance. $B_{I_{z}}$ is from the errors in the $I_{z}$ measurement introduced in Subsection 3.2. $B_{\rho}$ is from the water temperature-probe accuracy of $\pm 0.2{ }^{\circ} \mathrm{C} . B_{U_{\mathrm{C}}}$ is estimated end-to-end by calibrating the carriage speed. $B_{y_{\max }}, B_{\psi_{\max }}, B_{\beta}$ are associated with errors in the PMM setup and $B_{\omega}, B_{t}$ are determined from empirical estimations. $B_{F}$ is from the errors in the calibration and the volt-to-force conversion of the loadcell, and also from the errors in the model setup (such as the model misalignment or inaccurate drift angle setup at the PMM) and from the errors caused by the errors in the PMM motions (i.e., by $\left.B_{u}, B_{v}, B_{r}, B_{\dot{u}}, B_{\dot{v}}, B_{\dot{r}}\right)$. The full procedures of the bias 
limit estimations are presented in Simonsen (2004) and a summary is in the ITTC recommended procedures 7.5-02-06.04.

For the motion variables $r=z, \theta, \phi$, two elemental biases $B_{z_{\mathrm{mm}}}$ and $B_{L}$ for $z$ and $B_{\theta}$ and $B_{\phi}$ for $\theta$ and $\phi$ are identified from the DRE's (26) - (28). $B_{z_{\mathrm{mm}}}=0.9 \mathrm{~mm}$ is mainly from the unlevelness of the towing tank rail. The identification and evaluation of the rail bias is reported at the G2010 CFD Workshop (Larsson et al., 2012). $B_{\theta}=B_{\phi}=0.04^{\circ}$ are associated with errors in the Krypton motion tracker measurements as per Irvine et al. (2008).

\subsection{Precision limit}

Precision limits are determined with an end-to-end, multiple-test method, and computed with the standard multiple-test equation

$$
P_{r}=\frac{t \cdot s_{r}}{\sqrt{N}}
$$

where $t=2$ is the coverage factor for a $95 \%$ confidence level, and $s_{r}$ is the standard deviation of a sample of $N$ repeat realizations of the variable $r$. For the present study, $N=12$ repeat tests at the same conditions are obtained. The data set are spaced evenly in time (about 12 minutes) through the course of the experiments to account for factors that influence variability of the measurements, such as ambient motions in the tank water.

\subsection{Total uncertainty}

Total uncertainty is the RSS of bias $B_{r}$ and precision $P_{r}$ limits,

$$
U_{r}^{2}=B_{r}^{2}+P_{r}^{2}
$$

where the interval $\pm U_{r}$ contains the true value of $r$ at a $95 \%$ confidence level.

\subsection{UA results and asymmetry bias}

Table 9 presents a summary of UA results for static drift (top) and dynamic pure yaw (bottom) tests at $F r=0.280$. From the left side of Table 9, the bias and precision limits are presented as their contributions to the total uncertainty, $B_{r}^{2}$ and $P_{r}^{2}$ in percentages of $U_{r}^{2}$. The total uncertainty $U_{r}$ is presented in percentage of the absolute value, $|r|$, and the dynamic range, $D_{r}=$ $r_{\max }-r_{\min }$, of each variable for the static and dynamic test UA, respectively. For dynamic test, 
the UA results are time-varying, thus their time-mean values over one period, $\overline{B_{r}^{2}}, \overline{P_{r}^{2}}, \overline{U_{r}}$, are presented. The test cases presented herein are for the $\mathrm{FR}_{\mathrm{z} \theta}$ mount condition, thus no UA results are available for the roll $(\phi)$ variable.

For static drift test, $B_{r}^{2} \geq 95 \%$ and $P_{r}^{2} \leq 5 \%$ for all variables, indicating the DRE variables results are highly repeatable. The primary bias error source is identified as $B_{U_{\mathrm{C}}}$ for $X$ and $B_{F}$ for $Y$ and $N$, where $B_{F}$ is mainly originated from $B_{\beta}$, i.e., the errors in the model drift angle setting. $B_{L}, B_{T}, B_{\rho}$ contributions to $B_{r}$ are small or negligible. $U_{r}=1.9 \%, 3.4 \%, 2.8 \%$ for $X, Y, N$, respectively, which are considered reasonably small, however, larger than the resistance test uncertainty $U_{C_{T}}=0.67 \%$ of $C_{T}$ reported in the ITTC recommended procedure 7.5-02-02-02. For the motion variables, $U_{r}=10.0 \%$ and $26.4 \%$ for $z$ and $\theta$, which are relatively large but considered acceptable as the variables values are small.

For the dynamic pure yaw test, in general $\overline{B_{r}^{2}} \geq 75 \%$ and $\overline{P_{r}^{2}} \leq 25 \%$ for all variables except for $X$ of which $\overline{P_{r}^{2}}=81 \%$ is considerably larger than $\overline{B_{r}^{2}}=19 \%$. The highest contributing elemental bias is $B_{u}$ for $X$ and $B_{F}$ for $Y$ and $N$, of which the primary error source is $B_{U_{\mathrm{C}}}$ and $B_{\psi_{\text {max }}}$, respectively. $B_{L}, B_{T}, B_{x_{\mathrm{G}}}, B_{y_{\mathrm{G}}}, B_{m}, B_{I_{z}}$ and $B_{\rho}$ contributions to $B_{r}$ are small or negligible. $\overline{U_{r}}=7.6 \%, 3.7 \%, 1.2 \%$ for $X, Y, N$, respectively, and $20.1 \%$ and $35.7 \%$ for $z$ and $\theta$, respectively, which are relatively larger than or similar as those for the static drift UA.

Contrary to expectations, however, test results show large asymmetry, for example, between the positive and negative $\beta$ values of static drift test variables, where the discrepancy is larger than the $U_{r}$ limit estimations. Factors such as the model fabrication error and/or the initial heel of the model due in part to imperfect weight ballasting maybe the possible reasons. Due to lack of solid explanations, the average result $r_{\mathrm{m}}=\left(r^{+}+r^{-}\right) / 2$, where $r^{+}$and $r^{-}$are the $r$ values at positive and negative $\beta$, respectively, is taken as the representative data and the asymmetry is added to the total uncertainty as an asymmetry bias $B_{\text {asym }}$ defined as

$$
B_{\text {asym }}^{2}=D_{\text {asym }}^{2}-U_{r}^{2}
$$

if $D_{\text {asym }}>U_{r}$, where $D_{\text {asym }}=\left|r-r_{\mathrm{m}}\right|$, otherwise $B_{\text {asym }}=0$. Then, the total uncertainty $U_{r, \text { asym }}$ is the RSS of $B_{\text {asym }}$ and $U_{r}$ such as

$$
U_{r, \text { asym }}^{2}=U_{r}^{2}+B_{\text {asym }}^{2}
$$


For dynamic pure yaw test, on the other hand, the odd-order harmonics of the symmetric variables $X, z, \theta$ and the even-order harmonics of the anti-symmetric variables $Y, N$ (and $\phi$ ) are not expected from the FS expansions of the variables since the imposed PMM motions are symmetric about the model towing direction. Accordingly, $D_{\text {asym }}$ is redefined as $D_{\text {asym }}=\left|r-r_{\mathrm{FS}}\right|$, where $r_{\mathrm{FS}}$ is the FS reconstruction of $r$ with proper odd or even order FS harmonics, and $B_{\text {asym }}$ and $U_{r \text {,asym }}$ are as per (34) and (35), respectively. These symmetry considerations are also true for pure sway test data, but not appropriate for yaw-and-drift test data due to its asymmetric PMM motion.

The UA results are re-calculated by adding $B_{\text {asym }}$ to $U_{r}$ and the outcomes are presented in Table 9. Data asymmetry is large for $X$ and $\theta$ variables. For static drift, $B_{\text {asym }}=10.3 \%$ and $21.4 \%$ of $\left|r_{\mathrm{m}}\right|$, respectively. For pure yaw, $\overline{B_{\text {asym }}}=5.9 \%$ and $17.1 \%$ of $D_{r, \mathrm{FS}}$, respectively, where $D_{r, \mathrm{FS}}=r_{\mathrm{FS}, \max }-r_{\mathrm{FS}, \text { min }}$. Data asymmetry of $Y, N, \theta$ variables are small, $B_{\mathrm{asym}}=0$ for static drift and $\overline{B_{\text {asym }}}<2 \%$ for dynamic pure yaw. Accordingly, the resulting total uncertainty increases considerably for $X$, mildly for $z$, and small or zero for $Y, N, \theta$, with $U_{r \text {,asym }}=10.5 \%, 10.3 \%$, $3.3 \%, 2.8 \%, 28.5 \%$, respectively, for the static drift test and $\overline{U_{r, \text { asym }}}=10.4 \%, 20.1 \%, 4.6 \%$, $1.6 \%, 42.9 \%$, respectively, for the pure yaw test.

\subsection{UA comparisons between facilities and facility bias (or certification)}

UA results from three facilities are compared. In general, the UA show reasonable uncertainty levels for all facilities, nonetheless, in certain cases the deviation of data values between the facilities from their mean value exceeds the total uncertainty limit estimations. Those deviations are considered to be from differences in model size, model manufacture procedures, towing tank dimensions, water properties, towing carriage driving-mechanisms, PMM, measurement systems, and so on, which cannot be accounted for in individual facility UA procedures.

The facility biases or certification intervals of facilities are estimated using the $M \times N$ order testing method by Stern et al. (2005). The method is a statistical approach for assessing probabilistic confidence intervals for $M$ facilities and $N$ repeat tests with the mean facility data as reference values. The method assumes normal distribution for the sample population $X_{i}, 95 \%$ confidence level, $M \geq 10$, and $N \geq 10$. For the present example $M=3$ and $N=12$ are used. Although the number of facilities $M$ is small, the results show the usefulness of the approach as 
discussed in Stern et al. (2005). For the mean facility data $\bar{X}=\sum_{i} X_{i} / M$, where $X_{i}$ is $X, Y, N$ of an individual facility, the uncertainty $U_{\bar{X}}$ is the RSS of the bias limit $B_{\bar{X}}$ and the precision limit $P_{\bar{X}}$, which are the average RSS's of the $M$ bias limits $B_{i}$ and $M$ precision limits $P_{i}$, respectively. By comparing the difference $D_{i}=X_{i}-\bar{X}$ with its uncertainty $U_{D_{i}}=\left(U_{X_{i}}^{2}+U_{\bar{X}}^{2}\right)^{1 / 2}$ if $\left|D_{i}\right| \leq U_{D_{i}}$, then the individual facility is certified at interval $U_{D_{i}}$, whereas if $\left|D_{i}\right| \geq U_{D_{i}}$ the facility bias $U_{\mathrm{FB}_{i}}$ is defined as

$$
U_{\mathrm{FB}_{i}}^{2}=D_{i}^{2}-U_{D_{i}}^{2}
$$

with total uncertainty

$$
U_{\mathrm{T}_{i}}^{2}=U_{X_{i}}^{2}+U_{\mathrm{FB}_{i}}^{2}
$$

UA results from three facilities, IIHR, FORCE, and INSEAN, designated herein as facilities A, B, and C, respectively, are evaluated for facility bias. The facility biases and their comparisons are summarized in Table 10 for the static drift and dynamic pure yaw tests at $\mathrm{Fr}=$ 0.280, following the format and nomenclature used in Stern et al. (2005). The comparisons are made at $\beta=10^{\circ}$ for the static drift test UA and at $r=r_{\text {max }}$ for the pure yaw test UA. Table 10 provides the $N$-order and $M \times N$-order level UA's, which are each facilities UA and the facilitymean UA, respectively, and then the total uncertainties and facility certification (or bias). For the $\mathrm{N}$-order UA, the experimental data and UA of facilities B and C are recast to include asymmetry bias $B_{\text {asym }}$ as per equation (35) similarly as for facility A, thus $B_{X_{i}}^{2}=B_{r}^{2}+B_{\text {asym }}^{2}$, where $B_{r}$ is as per equation (32).

From Table 10, all three facilities are certified for static drift test (except for the $N$ variable of facility A) but at a large certification interval $U_{D_{i}}$ for the $X$ (average 10.1\%). A reduction of the interval of certification largely requires a reduction of individual facility biases for $X$ and carriage speed. For facility A, $D_{i}>U_{D_{i}}$ for $N$, thus the facility has a facility bias $U_{\mathrm{FB}_{i}}=2.7 \%$ and a total uncertainty $U_{T_{i}}=4.0 \%$ that is larger than the individual facility estimate $U_{X_{i}}=2.9 \%$. For the dynamic pure yaw test, all three facilities are certified for $X$ and $Y$ but at relatively large certification intervals (average $10.6 \%$ and $8.2 \%$, respectively). Facilities $\mathrm{B}$ and $\mathrm{C}$ have a facility bias $U_{\mathrm{FB}_{i}}=5.5 \%$ and $5.9 \%$ for $N$, respectively, thus a larger total uncertainty $U_{\mathrm{T}_{i}}=6.5 \%$ and $6.1 \%$, respectively, than the individual facility estimate $U_{X_{i}}=3.5 \%$ and $1.5 \%$, respectively. 
For identification of possible scale and $F r$ effects on the UA, all facilities $U_{\mathrm{T}_{i}}$ values are plotted together in Fig. 6 and for all $F r$ cases in Fig. 7 for (a) static drift test and (b) dynamic pure yaw test. For the former figure case, each facility uses a different scale model of length $L=$ $3.048 \mathrm{~m}, 4.0 \mathrm{~m}$, and $5.72 \mathrm{~m}$ for facility A, B, and C, respectively, and a normalized model length $L^{*}=L / 3.048 \mathrm{~m}=1.0,1.31$, and 1.88 , respectively, is used. As each facility also has different towing tank dimensions $(\mathrm{L} \times \mathrm{B} \times \mathrm{D}), 100 \mathrm{~m} \times 3.048 \mathrm{~m} \times 3.048 \mathrm{~m}, 240 \mathrm{~m} \times 12 \mathrm{~m} \times 4.4 \mathrm{~m}$, and 500 $\mathrm{m} \times 12.5 \mathrm{~m} \times 6.5 \mathrm{~m}$, for facilities $\mathrm{A}, \mathrm{B}$, and $\mathrm{C}$, respectively, the normalized model length $L^{*}$ represents the overall length scale of each facility. From Fig. $6, U_{\mathrm{T}}$ is seemingly less sensitive to the model scale change for the static drift case, where it shows a decreasing trend. Fig. 7 shows the trend of $U_{\mathrm{T}}$ with $F r$, which is similar as the model scale trend.

\section{RESULTS AND DISCUSSION}

\subsection{Forces and moment}

Static drift $X, Y$, and $N$ data are shown in Fig. 8. Data are fitted to quadratic functions for $X$ and cubic functions for $Y$ and $N$, such that $X=A+B \beta^{2}$ and $Y, N=A \beta+B \beta^{3}$, respectively. At $\beta=0^{\circ}$, the model is in a steady straight-ahead towing condition and the $X$ value is equivalent

to the total resistance coefficient, $C_{T}=R_{T} / \frac{1}{2} \rho U_{\mathrm{C}}^{2} S$, where $R_{T}$ is the total resistance and $S$ is the wetted-surface area of the model. The $X$ values at $\beta=0^{\circ}$ for $F r=0.138,0.280$, and 0.410 are converted into $C_{T 15 \mathrm{C}}$ that is the $C_{T}$ value with all data calibrated to a standard water temperature $15^{\circ} \mathrm{C}$ and compared with Longo and Stern (2005) in Fig. 9(a). Longo and Stern (2005) uses the same DTMB 5512 model and reports $C_{T 15 \mathrm{C}}$ values over a range of $F r=0.05 \sim 0.45$. The results of Longo and Stern (2005) show the trend of $C_{T 15 C}$ (thus, $X$ values at $\beta=0^{\circ}$ ) with $F r$ more clearly; first decreases for low $F r<0.25$, undulates for medium $F r<0.35$, then increases sharply for high $F r>0.35$. The present data set shows good agreement with Longo and Stern (2005) with all data points placed within the uncertainty limits of Longo and Stern (2005). Fig. 9(b) also shows the sinkage $\sigma$ and trim $\tau$ data and these will be discussed later in Section 6.4. Recalling Fig. 8 , for $\beta<10^{\circ}, X$ is less sensitive to $\beta$ and approximately a constant value; $Y$ and $N$ are nearly linear with $\beta$ with the slope $A$ of the curve-fit function seemingly independent of $F r$. These trends are consistent with Longo and Stern (2002) where measurements include resistance $C_{T}$, side force $C_{S}$, and drift moment $C_{M}$ coefficients (corresponding to $X, Y$, and $N$, respectively) 
of a Series 60 model in oblique towing for a range of $\beta=0^{\circ} \sim 10^{\circ}$ and $\mathrm{Fr}=0.1 \sim 0.35$. Longo and Stern (2002) curve-fits the data as $C_{T}=a \beta+b$ and $C_{S, M}=a \beta^{2}+b \beta+c$ and reports that the coefficients $a$ of $C_{T}$ and $b$ of $C_{S, M}$ are nearly independent of $F r$. For $\beta>10^{\circ}$, all data exhibit a non-linear trend with $\beta$. Typical values of the ratio of the non-linear term to the linear term of the curve-fit functions, $\lambda \equiv B \beta^{2} / A$, is $0.04,0.2$, and 0.7 , at $\beta=5^{\circ}, 10^{\circ}$, and $20^{\circ}$, respectively, where $N$ has a half to a third times smaller $\lambda$ values than $X$ or $Y$.

Time-histories of dynamic test data are complex-periodic with the PMM frequency $f$ in equations (12) and (13) as the fundamental frequency. Fig. 10 shows time-histories of which test conditions are $v_{\max }=0.174$ (or $\left.\beta_{\max }=10^{\circ}\right), r_{\max }=0.3$, and $\beta=10^{\circ}$ for pure sway, pure yaw, and yaw-and-drift tests at $F r=0.280$, respectively. The primary harmonic is the $2^{\text {nd }}$-order for the $X$ time-histories of pure sway and pure yaw with the amplitude $X_{2} / A=0.897$ and 0.564 , respectively, and the $1^{\text {st }}$-order for $Y$ and $N$ time-histories with $Y_{1} / A=0.998$ and 0.945 , respectively, and $N_{1} / A=0.970$ and 0.982 , respectively, where $A$ is the amplitude of the time-history fluctuation. The primary harmonic of the yaw-and-drift time-histories is the $1^{\text {st }}$-order commonly for all data variables with $X_{1} / A=0.925, Y_{1} / A=0.932$, and $N_{1} / A=0.986$. The phase of the primary harmonic of the pure sway, pure yaw, and yaw-and-drift $Y$ time-history is $\varphi_{1} / 2 \pi=0.085,0.281$, and 0.288 , respectively, and $N$ time-history $\varphi_{1} / 2 \pi=0.019,0.291$, and 0.282 , respectively. Although not shown in Fig. 10, these phase values are common among other test cases of each test type, varying only within about \pm 0.007 . The implication of these nearly constant phase values will be discussed in Section 6.2. The secondary harmonics are either the $4^{\text {th }}$ - or the $6^{\text {th }}$-order for the $X$ time-histories and the $3^{\text {rd }}$-order commonly for $Y$ and $N$ time-histories of pure sway and pure yaw time-histories. The magnitudes of the secondary harmonics are comparable with those of the primary harmonics for $X$ time-histories, whereas usually of only $3 \sim 6 \%$ of the primary harmonic amplitudes for $Y$ and $N$ time-histories. For yaw-and-drift time-histories the secondary harmonics are the $2^{\text {nd }}$-order for all data variables with the amplitudes generally about $10 \sim 20 \%$ of the primary harmonics amplitudes.

\subsection{Hydrodynamic derivatives}

The evaluation of the static derivatives $X_{v v}, Y_{v}, Y_{v v v}, N_{v}, N_{v v v}$ including the steady term $X_{*}$ in the mathematical models (1) - (3) is by curve-fitting the static drift data shown in Fig. 8 to 
the simplified mathematical models in equation (7); Table 11 presents the evaluation results. Fundamentally, the simplified mathematical models are equivalent with the quadratic and cubic curve-fit functions for $X, Y, N$ previously discussed in Section 6.1, by considering the relation$\operatorname{ship} v=-\sin \beta$, thus the same discussions of the polynomial coefficients $A$ 's and $B$ 's can be extended to those derivatives.

Evaluations of hydrodynamic derivatives from the dynamic tests are by using the harmonics form of the mathematical models in equation (8) (along with Table 1) and using either the 'Multiple-Run' methods (including $\mathrm{MR}_{\mathrm{L}}$ and $\mathrm{MR}_{\mathrm{H}}$ ) or the 'Single-Run' methods (including $\mathrm{SR}_{\mathrm{L}}$ and $\mathrm{SR}_{\mathrm{H}}$ ) introduced in Section 2.2. Figs. 11-13 show all the non-zero cosine and sine harmonics of equation (8), evaluated from the data time-histories of the entire test conditions at $\mathrm{Fr}$ $=0.280$, for pure sway, pure yaw, and yaw-and-drift tests, respectively. Table 11 presents the evaluation outcomes by the $\mathrm{MR}_{\mathrm{L}}$ and $\mathrm{MR}_{\mathrm{H}}$ methods.

\section{Multiple-Run (MR) method:}

Although the static drift test is one of the most commonly used experimental methods obtaining the aforementioned sway-velocity derivatives, those derivatives are also attainable from the pure sway test. Derivatives $X_{*}$ and $X_{v v}$ are determined by curve-fitting the $X_{0}$ harmonic data, $Y_{v}$ and $Y_{v v v}$ the $Y_{\mathrm{C} 1}$ harmonic data, and $N_{v}$ and $N_{v v v}$ the $N_{\mathrm{C} 1}$ harmonic data shown in Fig. 10(a)(c). The quadratic and cubic trends of the $X_{0}$ harmonic and $Y_{\mathrm{C} 1}$ and $N_{\mathrm{C} 1}$ harmonics data resemble those of the static drift $X$ data and $Y$ and $N$ data. However, compared to the static drift data case $\left(\lambda \approx 0.2\right.$ at $\left.\beta=10^{\circ}\right)$, the pure sway harmonics data exhibit a larger non-linearity in their curvefits, with the ratio $\lambda \approx 0.25$ at $v_{\max }=0.174$ (i.e., at $\beta_{\max }=10^{\circ}$ ), where $\lambda \equiv\left(X_{v v} / 2 X_{*}\right) \cdot v_{\max }^{2}$, $\left(3 Y_{v v v} / 4 Y_{v}\right) \cdot v_{\max }^{2}$, and $\left(3 N_{v v v} / 4 N_{v}\right) \cdot v_{\max }^{2}$, for $X_{0}, Y_{\mathrm{C} 1}$, and $N_{\mathrm{C} 1}$, respectively, defined similarly as discussed earlier in Section 6.1. The values of linear derivatives, $X_{*}, Y_{v}$, and $N_{v}$, evaluated from the pure sway test in general agree well with those from the static drift test with a ratio of $1.02,0.88$, and 1.01, respectively. On the other hand, the values of non-linear derivatives, $X_{v v}$, $Y_{v v v}$, and $N_{v v v}$, from the pure sway test are relatively larger than those from the static drift test with a ratio of $3.12,1.53$, and 1.30 , respectively. Sway-acceleration derivatives $Y_{\dot{v}}$ and $N_{\dot{v}}$ are determined from linear curve-fits of $Y_{\mathrm{S} 1}$ and $N_{\mathrm{S} 1}$ data shown in Fig. 11(c). Knowing that the harmonics $Y_{\mathrm{S} 1}$ and $N_{\mathrm{S} 1}$ stem from the acceleration (or the added-mass) terms and $Y_{\mathrm{C} 1}$ and $N_{\mathrm{C} 1}$ from the velocity (or the damping) terms of the mathematical models in equations (1)-(3), their 
ratios may be of interest. For example, the ratio $Y_{\mathrm{S} 1} / Y_{\mathrm{C} 1}$ is the tangent of the $1^{\text {st }}$-order harmonic phase of $Y$ time-history, i.e., $\tan \varphi_{1}=Y_{\dot{v}} \omega / Y_{v}(1+\lambda)$. For $\beta_{\max }=10^{\circ}$, the phase value calculated from this ratio is $\varphi_{1} / 2 \pi=0.084$ for $Y$ and 0.020 for $N$, which are close to the actual phase values for pure sway $Y$ and $N$ time-histories discussed in Section 6.1. Since the derivatives $Y_{\dot{v}}$ and $Y_{v}$ or $N_{\dot{v}}$ and $N_{v}$ are constant values for a given hull form and a given $F r, \varphi_{1}$ is approximately only dependent on the sway frequency $\omega$ if $\lambda \ll 1$. This may explain the reason for the nearly constant phase of the primary harmonics from the data time-histories of different test conditions.

Rotary derivatives $X_{r r}, Y_{r}, Y_{r r r}, N_{r}, N_{r r r}$ and acceleration derivatives $Y_{\dot{r}}$ and $N_{\dot{r}}$ are determined from curve-fits of the harmonics $X_{0}, Y_{\mathrm{S} 1}, Y_{\mathrm{C} 1}, N_{\mathrm{S} 1}$, and $N_{\mathrm{C} 1}$ data shown in Fig. 12(a)(c). The goodness of the data-fits seems adequate demonstrating the validity of the mathematical models. The harmonics $X_{0}, Y_{\mathrm{S} 1}$, and $N_{\mathrm{S} 1}$ of the pure yaw tests also show similar quadratic and cubic trends as the pure sway $X_{0}, Y_{\mathrm{C} 1}$, and $N_{\mathrm{C} 1}$ harmonics and the static drift $X$ data and $Y$ and $N$ data. However, the pure yaw harmonics data exhibit a weaker non-linearity in their curve-fits, with the ratio $\lambda \approx 0.07$ at $r_{\max }=0.3\left(\right.$ with $\left.\psi_{\max }=10.2^{\circ}\right)$ than 0.2 of the static drift data at $\beta=$ $10^{\circ}$ and 0.25 of the pure sway harmonics at $\beta_{\max }=10^{\circ}$. Here, $\lambda \equiv\left(X_{r r} / 2 X_{*}\right) \cdot r_{\max }^{2}$, $\left(3 Y_{r r r} / 4 Y_{r}\right) \cdot r_{\max }^{2}$, and $\left(3 N_{r r r} / 4 N_{r}\right) \cdot r_{\max }^{2}$, for $X_{0}, Y_{\mathrm{C} 1}$, and $N_{\mathrm{C} 1}$. Yaw-acceleration derivatives $Y_{\dot{r}}$ and $N_{\dot{r}}$ are determined from linear curve-fits of $Y_{\mathrm{C} 1}$ and $N_{\mathrm{C} 1}$ data shown in Fig. 12(c). Similarly as for pure sway data, the ratio $Y_{\mathrm{S} 1} / Y_{\mathrm{C} 1}$ is the tangent of the $1^{\text {st }}$-order harmonic phase, $\tan \varphi_{1}=-Y_{r}(1+\lambda) / Y_{\dot{r}} \omega$. For $r_{\max }=0.3, \varphi_{1} / 2 \pi=0.295$ for $Y$ and 0.285 for $N$, which are close to the actual phase values discussed in Section 6.1.

Sway-yaw coupled derivatives $X_{v r}, Y_{v r r}, Y_{r v v}, N_{v r r}$, and $N_{r v v}$ are determined by curvefitting the yaw-and-drift harmonics $X_{\mathrm{S} 1}, Y_{0}, Y_{\mathrm{S} 1}, N_{0}$, and $N_{\mathrm{S} 1}$, respectively, which are shown in Fig. 13(a)-(c). The curve-fits for $Y_{v r r}$ and $N_{v r r}$ require sway-velocity derivatives $Y_{v}, Y_{v v v}, N_{v}$, $N_{v v v}$ and the curve-fits for $Y_{r v v}$ and $N_{r v v}$ require the yaw-rate derivatives $Y_{r}, Y_{r r r}, N_{r}, N_{r r r}$ for $Y_{r v v}$ as input, for which those derivatives of the static drift test are used for the former and the derivatives of the pure yaw test for the latter curve-fits.

Non-linear static $X_{v v}, Y_{v v v}, N_{v v v}$ and rotary $X_{r r}, Y_{r r r}, N_{r r r}$ derivatives and the crosscoupled derivatives $Y_{v r r}$ and $N_{v r r}$ are as well attainable by applying the $\mathrm{MR}_{\mathrm{H}}$ method to the higher order harmonics of dynamic test data. Nonetheless, those derivatives values by the $\mathrm{MR}_{\mathrm{H}}$ method usually show disagreements with the values by the $\mathrm{MR}_{\mathrm{L}}$ method. For example, the value of $X_{v v}$ by the $\mathrm{MR}_{\mathrm{H}}$ method is nearly a quarter of the value by the $\mathrm{MR}_{\mathrm{L}}$ method. Similarly, the 
values of $X_{r r}$ by the $\mathrm{MR}_{\mathrm{H}}$ method are only about one-half to one-fifth of the values by the $\mathrm{MR}_{\mathrm{L}}$ method. One possible reason for this disparity, particularly for those $X_{v v}$ and $X_{r r}$ derivatives, is that the mathematical modeling in equations (1)-(3) uses the Taylor Series expansions only up to the $3^{\text {rd }}$-order. Thus, the mathematical models for $X_{0}$ (See Table 1) include polynomial terms only up to the $2^{\text {nd }}$-order, for example, $X_{0}=X_{*}+\frac{1}{2} X_{v v} v_{\max }^{2}$ for pure sway. However, as discussed previously, the $4^{\text {th }}$ - or $6^{\text {th }}$-order harmonics of $X$ time-histories are comparable in magnitudes with the $2^{\text {nd }}$-order harmonic. This implies that the actual $X_{0}$ value from measurements may include contributions from the higher-order harmonics such that $X_{0}=X_{*}+\frac{1}{2}\left(X_{v v} \cdot \eta\right) v_{\max }^{2}$, where $\eta=1+{ }_{4}^{3} X_{v v v v} v_{\max }^{2} / X_{v v}+\frac{5}{8} X_{v v v v v v} v_{\max }^{4} / X_{v v}+\cdots$ by including more and more higher-order terms in the Taylor Series expansion. As a result, it is possible that the $\mathrm{MR}_{\mathrm{L}}$ method overevaluates $X_{v v}$ by a factor $\eta$ (and similarly $X_{r r}$ as well). The fact that the $X_{v v}$ value by the $\mathrm{MR}_{\mathrm{H}}$ method agrees better with the value by the static drift test may support this hypothesis. The values of the other derivatives by the $\mathrm{MR}_{\mathrm{H}}$ method are usually lager than the values from the $M R_{L}$ method, by a factor of about $1 \sim 3$, with only a few exceptions.

\section{Single-Run (SR) method:}

Fig. 14 shows hydrodynamic derivative ratios using the SR and $\mathrm{MR}_{\mathrm{L}}$ methods. For example, the symbols shown at $\beta_{\max }=10^{\circ}$ in Fig. 14(a) represents the ratios of the sway derivatives from a single-run of pure sway at $\beta_{\max }=10^{\circ}$ to those derivatives by the $\mathrm{MR}_{\mathrm{L}}$ method. The closer the ratio value is to one, the better the agreement between the two methods. The steady term $X_{*}$ and the linear derivatives $Y_{v}, N_{v}, Y_{\dot{v}}$, and $N_{\dot{v}}$ by the $\mathrm{SR}_{\mathrm{L}}$ method agree well with those by the $\mathrm{MR}_{\mathrm{L}}$ method for all $\beta_{\max }$ cases with an average ratio of 1.03 . Whereas the non-linear derivatives $X_{v v}, Y_{v v v}$, and $N_{v v v}$ values by the SR method are greater than those by the $\mathrm{MR}_{\mathrm{L}}$ method, with an average ratio of $1.28,1.47$, and 5.80, respectively. Particularly, the $N_{v v v}$ by the SR method for $\beta_{\max }=2^{\circ}$ is more than 10 times larger than by the $\mathrm{MR}_{\mathrm{L}}$ method; the average ratio excluding this case is 2.25. Yaw derivatives in Fig. 14(b) also show similar trends. The linear rotary $\left(Y_{r}, N_{r}\right)$ and acceleration $\left(Y_{\dot{r}}, N_{\dot{r}}\right)$ derivatives by the $\mathrm{SR}_{\mathrm{L}}$ method in general agree well with those by the $\mathrm{MR}_{\mathrm{L}}$ method. The average ratio value is about 0.7 for $Y_{r}$ and $Y_{\dot{r}}$ and about 1.0 for $N_{r}$ and $N_{\dot{r}}$. When $r_{\max }$ is smaller than 0.2 , the ratio values are very large for the non-linear rotary derivatives $X_{r r}, Y_{r r r}$, and $N_{r r r}$, almost up to 50 or even higher. By excluding those small $r_{\max }$ 
cases, the average ratio value is $0.39,3.35$, and 1.01 for $X_{r r}, Y_{r r r}$, and $N_{r r r}$, respectively. The sway-yaw-coupled derivatives $X_{v r}, Y_{v r r}, N_{v r r}, Y_{r v v}$, and $N_{r v v}$ by the $\mathrm{SR}_{\mathrm{L}}$ method agree well with those by the $\mathrm{MR}_{\mathrm{L}}$ method for all the $\beta$ cases with an average ratio of 1.02 . For $Y_{v r r}$ and $N_{v r r}$, the $\mathrm{SR}_{\mathrm{H}}$ method is applicable. The $Y_{v r r}$ values by the $\mathrm{SR}_{\mathrm{H}}$ method are also close to those by the $\mathrm{MR}_{\mathrm{L}}$ method with an average ratio value of 1.04 , whereas the values of $N_{v r r}$ by the $\mathrm{SR}_{\mathrm{H}}$ method are relatively large with an average ratio value of 1.58 .

\section{Reconstruction error:}

Given the four different evaluation methods, i.e., $\mathrm{MR}_{\mathrm{L}}, \mathrm{MR}_{\mathrm{H}}, \mathrm{SR}_{\mathrm{L}}$, and $\mathrm{SR}_{\mathrm{H}}$, the assessment of their performance is evaluated by defining and examining the errors in reconstructing the forces and moment time-histories. Reconstructions are implemented using the mathematical model in equations (1)-(3) or the harmonics form in equation (8). Then, a reconstruction error is defined as $E_{R}(\%)=\int_{0}^{T}|D(t)-R(t)| d t / \int_{0}^{T}|D(t)| d t$, where $D(t)$ is the measured and $R(t)$ is the reconstructed time-histories and $T$ is the period of one PMM cycle. Herein the reconstruction cases are named with the same name as the reconstruction method, e.g., $\mathrm{MR}_{\mathrm{L}}, \mathrm{SR}_{2^{\circ}}$, or $\mathrm{SR}_{0.15}$. The subscripts of the SR reconstruction cases represent the origin of the hydrodynamic derivatives. For example, the $\mathrm{SR}_{2}$ case of pure sway indicates that reconstructions are by using the hydrodynamic derivatives obtained from the $\beta_{\max }=2^{\circ}$ case, the $\mathrm{SR}_{0.15}$ case of pure yaw from the $r_{\max }=0.15$ case, and so on. For $\mathrm{MR}_{\mathrm{H}}$ or $\mathrm{SR}_{\mathrm{H}}$ cases only the non-linear derivatives are available, thus the reconstructions use the linear derivatives by the $\mathrm{MR}_{\mathrm{L}}$ and $\mathrm{SR}_{\mathrm{L}}$ method, respectively.

Table 12 reports the average error $\overline{E_{R}}(\%)$, which is the average value of the $E_{R}(\%)$ 's while constructing each of $\beta_{\max }=2^{\circ}, 4^{\circ}$, and $10^{\circ}$ cases for pure sway, $r_{\max }=0.05,0.15,0.30$, $0.45,0.60$, and 0.75 cases for pure yaw, and $\beta=9^{\circ}, 10^{\circ}$, and $11^{\circ}$ cases for yaw-and-drift. For most of the cases, the smallest error is for the $\mathrm{MR}_{\mathrm{L}}$ cases with $\overline{E_{R}}(\%)=3 \sim 9,5 \sim 17$, and $3 \sim 11$ for pure sway, pure yaw, and yaw-and-drift, respectively. The largest error is the $\mathrm{SR}_{2}{ }^{\circ}$ and $\mathrm{SR}_{0.15}$ case of pure sway and pure yaw reconstructions, where $\overline{E_{R}}(\%)=5 \sim 30$ and $106 \sim 616$, respectively. The $\overline{E_{R}}(\%)$ of the SR cases tend to become comparable to those of the $\mathrm{MR}_{\mathrm{L}}$ cases as $\beta_{\max }$ or $r_{\max }$ increases. For yaw-and-drift reconstructions $\overline{E_{R}}(\%)=3 \sim 11$ commonly for all cases. Consequently, the $\mathrm{MR}_{\mathrm{L}}$ method in general gives smaller reconstruction errors than the other me- 
thods and the SR method gives comparable reconstruction errors with the $M R_{L}$ method when the motion parameter values are sufficiently large.

\section{Surge-coupled derivatives:}

Evaluations of the sway-surge-coupled and yaw-surge-coupled derivatives are done by using the static and rotary derivatives obtained from various $\mathrm{Fr}$ cases. Fig. 15 shows the trend of those derivatives with the speed change, $\Delta u=(u-U) / U$, where $u$ is the surge velocity corresponding to each of $F r=0.138,0.280$, and 0.410 and $U$ to $F r=0.280$; thus $\Delta u=-0.5,0.0$, and 0.5 , respectively. Also, Fig. 15 presents each derivative value of different $F r$ cases relative to the value of the $F r=0.280$ case, thus the ratio value at $\Delta u=0$ in Fig. 15 is always unity for all derivatives cases. The rate of change of each derivative with $\Delta u$ is assessed by using a percentchange variable defined as $\Delta(\%) \equiv\left(x_{\Delta u=0.5}-x_{\Delta u=-0.5}\right) / x_{\Delta u=0} \times 100$, where $x_{\Delta u=-0.5,0,0.5}$ is any derivative $x$ value at $\Delta u=-0.5,0$, and 0.5 , respectively.

From Fig. 15(a), the change of $X_{*}$ with $\Delta u$ is equivalent with the ship resistance change with speed as discussed earlier in Section 6.1. The linear derivatives $Y_{v}$ and $N_{v}$ are less sensitive to the speed change, with $\Delta(\%)=11$ and 19 , respectively. Whereas the changes of the non-linear derivatives $X_{v v}, Y_{v v v}$, and $N_{v v v}$ are substantial with $\Delta(\%)=81,111$, and 156, respectively. From Fig. 15(b), the changes of the linear derivatives $Y_{r}$ and $N_{r}$ are relatively small with $\Delta(\%)=56$ and 38, respectively, while the the changes of the non-linear derivatives $X_{r r}, Y_{r r r}, N_{r r}$ are large with $\Delta(\%)=109,75$, and 121 , respectively. The derivatives values are curve-fitted to a $2^{\text {nd }}$-order polynomial functions of $\Delta u$, then surge-coupled derivatives are the $1^{\text {st }}$ and $2^{\text {nd }}$ differentiations of the fit-functions evaluated at $\Delta u=0$. Table 11 presents the results.

\subsection{Comparisons between facilities}

Hydrodynamic derivatives values are compared among three different facilities. All the facilities used the same test matrix shown in Table 6 with proper scaling based on each facility model size for the same non-dimensional test-parameter values including the same drift angle values for static drift tests and the same non-dimensional cyclic frequency values for the dynamic tests. The only exception was the $\dot{r}_{\text {max }}$ values of pure yaw tests for the $F r=0.280$ case, where the FORCE facility used about $0.7 \sim 1.0$ and the INSEAN facility about $0.85 \sim 1.0$ of the IIHR facility's $\dot{r}_{\max }$ values, respectively, by using non-dimensional cyclic frequency $\left(\omega_{1}^{\prime}, \omega_{2}^{\prime}, \omega_{3}^{\prime}\right)$ val- 
ues about 0.8 1.0 and 0.9 1.0, respectively, of the IIHR frequency values. Simonsen (2004) and Benedetti et al. (2007) provides the full test matrix for FORCE and INSEAN, respectively. All the tests were done for $\mathrm{FR}_{\mathrm{z} \theta}$ mount condition and the hydrodynamic derivatives were evaluated by using the $\mathrm{MR}_{\mathrm{L}}$ method commonly for all three facilities. Flow Reynolds number was $R e=4.8$ $\times 10^{6}, 6.3 \times 10^{6}$, and $1.2 \times 10^{7}$ for IIHR, FORCE, and INSEAN facility, respectively, based on each facility water temperature $21.5,15.7$, and $21.55^{\circ} \mathrm{C}$, respectively, and each facility towing speed corresponding to $\mathrm{Fr}=0.280$.

Facility comparisons use a regression analysis and then a correlation analysis to examine possible relationships between the hydrodynamic derivatives with model size. The analysis and nomenclatures follow Sec. 23.9 and Sec. 23.10 in Kreyszig (1999). The approach is a standard linear regression to define a sample linear regression line, $y=k_{0}+k_{1} x$, where $x$ is the independent (or the control) variable and $y$ is the dependent variable of the regression, and the slope of the line $k_{1}$ is the regression coefficient. The assessment of the confidence interval $K$ for $\kappa_{1}$ is at $95 \%$ level, where $\kappa_{1}$ is the theoretical counterpart of $k_{1}$. The method for the coefficient estimation is an ordinary least squares. The sample correlation coefficient is $r=s_{x y} / s_{x} s_{y}$, where $s_{x y}$ is the sample covariance and $s_{x}^{2}$ and $s_{y}^{2}$ are the sample variances. The correlation coefficient range is $-1 \leq r \leq 1$; a value of 1 indicates a perfect positive relationship and -1 a perfect negative relationship between $x$ and $y$, and a value of 0 indicates no correlation between the variables. For the latter case, a test for the hypothesis $\rho=0$ against the alternative $\rho>0$ is done at a significance level of 5\%, where $\rho$ is the theoretical counterpart of $r$.

Fig. 16 shows the hydrodynamic derivatives of all three facilities. All derivatives data are scaled with the facility-mean values. The dimensionless model length $L^{*}=1.0,1.31$, and 1.88 (defined in Section 5.6) represents each facility and the model size. Herein the analysis focuses on the general trends of the derivatives after grouping them by type. A total of 6 groups are constructed, which are the linear $\left(Y_{v}\right.$ and $\left.N_{v}\right)$ and non-linear $\left(X_{v v}, Y_{v v v}\right.$, and $\left.N_{v v v}\right)$ static derivatives groups, the linear $\left(Y_{r}\right.$ and $\left.N_{r}\right)$ and non-linear $\left(X_{r r}, Y_{r r r}, N_{r r r}\right)$ rotary derivatives groups, and the acceleration $\left(Y_{\dot{v}}\right.$ and $\left.N_{\dot{v}}\right)$ and $\left(Y_{\dot{r}}\right.$ and $\left.N_{\dot{r}}\right)$ derivatives groups. The sample size of each group is $n$ $=18,27,18,27,6$, and 18, respectively. The dashed-lines in each figure represent the regression line of each group. The groups $\left(Y_{v}\right.$ and $\left.N_{v}\right)$ and $\left(X_{v v}, Y_{v v v}\right.$, and $\left.N_{v v v}\right)$ in Fig. 16(a) exhibit almost flat regression lines with $k_{1}=0.006$ and -0.027 , respectively, for which $K=0.055$ and 0.235 , 
respectively. The correlation coefficient values are close to zero for these groups, $r=0.06$ and 0.05 , respectively. The hypothesis of $\rho=0$ is accepted for these groups. The groups $\left(Y_{r}\right.$ and $\left.N_{r}\right)$ and $\left(X_{r r}, Y_{r r r}\right.$, and $\left.N_{r r r}\right)$ in Fig. 16(b) exhibit mildly sloping regression lines with $k_{1}=0.140$ and -0.158 , respectively, for which $K=0.157$ and 0.457 , respectively. The correlation coefficient values for these groups are $r=0.43$ and -0.14 , respectively. The hypothesis of $\rho=0$ is rejected for the former group and accepted for the latter group. The groups $\left(Y_{\dot{v}}\right.$ and $\left.N_{\dot{v}}\right)$ and $\left(Y_{\dot{r}}\right.$, and $\left.N_{\dot{r}}\right)$ in Fig. 16(c) exhibit a mildly sloping and a steep regression line, respectively, with $k_{1}=-0.130$ and -0.517 , respectively, for which $K=0.121$ and 0.127 , respectively. The correlation coefficient values are close to \pm 1 for these groups, $r=-0.83$ and 0.91 , respectively. The hypothesis of $\rho=0$ is rejected for these groups.

The correlation analysis indicates that the linear and non-linear static derivatives and the non-linear rotary derivatives are independent of model scale. On the other hand, the linear rotary derivatives and the acceleration derivatives show a correlation with model scale. Also, the regression analysis expects generally a mild increase of the $Y_{r}$ and $N_{r}$ derivatives, a mild decrease of the $Y_{\dot{v}}$ and $N_{\dot{v}}$ derivatives, and a rather large increase of the $Y_{\dot{r}}$ and $N_{\dot{r}}$ derivatives as model size increases.

\subsection{Heave, pitch, and roll motions}

Static drift $z, \theta$, and $\phi$ are shown in Fig. 17(a)-(c). In the figures, the hollow and greyfilled symbols signify different model mounting conditions $\mathrm{FR}_{\mathrm{z} \theta}$ and $\mathrm{FR}_{\mathrm{z} \theta \phi}$, respectively. At $\beta=$ $0^{\circ}, z$ increases with $F r$ in a monotonic fashion, whereas $\theta$ initially decreases to a negative value then increases to a positive value. The dynamic sinkage, $\sigma=(\Delta \mathrm{FP}+\Delta \mathrm{AP}) / 2 L$, and trim, $\tau=$ $(\Delta \mathrm{AP}-\Delta \mathrm{FP}) / L$, data of Longo and Stern (2005) in Fig. 9(b) show this trend more clearly, where $\Delta \mathrm{FP}$ and $\triangle \mathrm{AP}$ are the vertical displacements of the model at the forward- and aftperpendicular, respectively. The relationship between the variables is $\Delta \mathrm{FP}=z-(L / 2) \sin \theta$ and $\Delta \mathrm{AP}=z+(L / 2) \sin \theta$, hence $\sigma=z$ and $\tau=\sin \theta$. From Fig. 9(b), $\sigma$ increases monotonically from low to high $F r$, whereas $\tau$ first decreases to negative (bow down) for low $F r<0.3$, oscillates at medium $\mathrm{Fr}<0.35$, then increases sharply to positive (bow up) at high $\mathrm{Fr}$.

For $\beta>0^{\circ}$ in Fig. $17, z$ and $\theta$ data fit well to quadratic, $(z, \theta)=A+B \beta^{2}$, and $\phi$ data to cubic, $\phi=A \phi+B \phi^{3}$, functions as predicted by equations (9) and (10). The curve-fit coefficients $A$ and $B$ are summarized in Table 13. The $A$ values of $z$ and $\theta$ correspond to the $z$ and $\theta$ at 
$\beta=0^{\circ}$ which are discussed above. The $B$ values of $z$ and $\theta$ increase and decrease, respectively, with $F r$. Provided $B^{*} \equiv B / B_{0}$ and $F r^{*} \equiv F r / F r_{0}$ such that $B_{0}$ is the $B$ value at $F r=0.280$ and $F r_{0}=0.280$, then $B^{*} \approx\left(F r^{*}\right)^{n}$, thus $B \sim a \cdot F r^{n}$, where $a>0$ and $n \approx 2.5$ for $z$ and $a<0$ and $n$ $\approx 1$ for $\theta$, commonly for $\mathrm{FR}_{z \theta}$ and $\mathrm{FR}_{z \theta \phi}$. Similarly, the $A$ of $\phi$ shows a tendency with $F r$ as $A \sim$ $a \cdot F r+b \cdot F r^{n}$, where $n \approx 3$. However, $B$ of $\phi$ shows no clear tendency with $F r$ from the present data. The $\phi$ of $\mathrm{FR}_{z \theta}$ is trivial and the data are not analyzed. When the difference between the two different mount conditions is defined as $\zeta_{\phi} \equiv x_{z \theta \phi} / x_{z \theta}$, where $x_{z \theta}$ and $x_{z \theta \phi}$ are any variable $x$ of $\mathrm{FR}_{z \theta}$ and $\mathrm{FR}_{z \theta \phi}$, respectively, in general $\zeta_{\phi} \approx 1.2$ and 0.9 for the $A$ 's and $B$ 's of $z$, respectively, and $\zeta_{\phi} \approx 0.1 \sim 1.1$ and $4 \sim 12$ for the $A$ 's and $B$ 's of $\theta$, respectively. These $\zeta_{\phi}$ values indicate that the effect of the roll motion on heave is small and that the effect on pitch motion is significant particularly as drift angle becomes large, which are evident from Fig. 17(a) and (b).

Typical time-histories of dynamic $z, \theta$, and $\phi$ are shown in Fig. 18(a) - (c) for $\mathrm{FR}_{z \theta}$, respectively, and in Fig. 18(d) - (f) for $\mathrm{FR}_{z \theta \phi}$, respectively. The $z$ and $\theta$ time-histories of pure sway and pure yaw are mainly of the $2^{\text {nd }}$-order harmonic with $\mathrm{A}_{2} / \mathrm{H}=0.98 \sim 1.01$, where $\mathrm{A}_{2}$ is the $2^{\text {nd }}$ order harmonic amplitude and $\mathrm{H}$ is the peak amplitude (a half of the peak-to-peak amplitude) of the time-history oscillation. The secondary harmonics are the $4^{\text {th }}$ - and the $6^{\text {th }}$-order with $\mathrm{A}_{4} / \mathrm{H}=$ $0.05 \sim 0.13$ and $\mathrm{A}_{6} / \mathrm{H}=0.01 \sim 0.07$. The $\phi$ time-histories of pure sway and pure yaw are mainly of the $1^{\text {st }}$-order harmonic with $\mathrm{A}_{1} / \mathrm{H}=0.96 \sim 1.01$ and the secondary harmonics are the $3^{\text {rd }}$ - and the $5^{\text {th }}$-order with $\mathrm{A}_{3} / \mathrm{H}=0.06 \sim 0.09$ and $\mathrm{A}_{5} / \mathrm{H}=0.01 \sim 0.06$. For yaw-and-drift, all of the $z, \theta$, and $\phi$ time-histories are commonly of the $1^{\text {st }}$-order harmonic with $\mathrm{A}_{1} / \mathrm{H}=0.97 \sim 1.03$ and the secondary harmonic is the $2^{\text {nd }}$-order with $\mathrm{A}_{2} / \mathrm{H}=0.12 \sim 0.14$ for $z$ and $\theta$ and $0.01 \sim 0.08$ for $\phi$, respectively, and all the other harmonics are negligibly small with $\mathrm{A}_{3,4,5,6} \mathrm{H}<0.1$. Again, the $\phi$ time-histories of $\mathrm{FR}_{\mathrm{z} \theta}$ are trivial and the data are not analyzed.

The major harmonics of the pure yaw $z, \theta$, and $\phi$ time-histories are shown in Fig. 19 for all $r_{\text {max }}$ and $F r$ test cases of $\mathrm{FR}_{z \theta}$ and $\mathrm{FR}_{z \theta \phi}$. For $\mathrm{FR}_{z \theta}$, the static component of $z$ and $\theta$ timehistories, $z_{0}$ and $\theta_{0}$, respectively, are quadratic with $r_{\max }$ such that $\left(z_{0}, \theta_{0}\right)=A+B r_{\max }^{2}$, as expected from the particular solutions (summarized in Table 4 ) of the heave and pitch motion equations (4) and (5). Table 14 presents the $A$ and $B$ values for all $F r$ cases. The $A$ 's of $z_{0}$ and $\theta_{0}$ are the same as the static drift $z$ and $\theta$ at $\beta=0^{\circ}$. The $B$ 's of $z_{0}$ show a tendency with $F r$ as $B \sim F r^{n}$, where $n \approx 3.5$. However, the $B$ 's of $\theta_{0}$ show a non-monotonic tendency with $F r$ and their values 
first increase then decrease as $F r$ becomes larger. The $2^{\text {nd }}$-order harmonic $z_{2}$ and $\theta_{2}$ are also quadratic with $r_{\max }$ such that $\left(z_{2}, \theta_{2}\right)=C_{0}+C r_{\max }^{2}$, where the $C_{0}$ and $C$ values are also presented in Table 14. The constant term $C_{0}$ 's of both $z_{0}$ and $\theta_{0}$ are not expected from the particular solutions. Those terms are considered as originated from the transient oscillation of the model as discussed in Section 4.1. The frequency of the transient motion $f_{\mathrm{tr}} \approx 0.25 \mathrm{~Hz}$ is coincident with the $2^{\text {nd }}$-order harmonic frequency $2 f=0.268 \mathrm{~Hz}$. The tendency of the $B$ 's of $z_{2}$ and $\theta_{2}$ with $F r$ is $B$ $\sim F r^{n}$, where $n \approx 3.5$ for $z_{2}$ and $n \approx 1$ for $\theta_{2}$. For Fig. 19(a), (b), and (d), the $z_{0}, z_{2}$, and $\theta_{0}$ of $\mathrm{FR}_{z \theta \phi}$ are close to those of $\mathrm{FR}_{z \theta}$ with $\zeta_{\phi}=0.5 \sim 1$.4. However, the $\theta_{2}$ 's of $\mathrm{FR}_{z \theta \phi}$ in Fig. 19(e) is considerably larger than those of $\mathrm{FR}_{z \theta}$ with $\zeta_{\phi}=2 \sim 10$, which indicates that the effect of roll motion is mainly on the dynamic component of pitch motion and is less significant to heave motions. Lastly, from Fig. 19(a) and (f), $\phi_{1}=0.2^{\circ}, 0.9^{\circ}$, and $2.1^{\circ}$, and $\phi_{3}=0.003^{\circ}, 0.011^{\circ}$, and $0.098^{\circ}$ at $F r=0.138,0.280$, and 0.410 , respectively. The tendency with $F r$ is $\left(\phi_{1}, \phi_{3}\right) \sim F r^{n}$, where $n \approx 2$ and 5.5 , respectively.

\subsection{The effects of motions on forces and moment}

Test results by using four different mount conditions, $\mathrm{FX}_{0}, \mathrm{FX}_{\sigma \tau}, \mathrm{FR}_{z \theta}$, and $\mathrm{FR}_{z \theta \phi}$, are compared and the effects of the ship motions in the vertical plane on the forces and moment components in the horizontal plane are discussed and subsequently their effects on the hydrodynamic derivatives. The $\mathrm{FX}_{0}$ condition conforms strictly to the $3 \mathrm{DOF}$ assumption of the mathematical modeling in equations (1)-(3) by constraining the ship model motions in heave, pitch, and roll. The $\mathrm{FX}_{\sigma \tau}$ condition is also in $3 \mathrm{DOF}$ but imposes a perturbation to $\mathrm{FX}_{0}$ in heave and pitch for a fixed amount corresponding to dynamic sinkage $\sigma$ and trim $\tau$ in straight-ahead running. The $\mathrm{FR}_{z \theta}$ is the most typical mounting condition in standard PMM procedures. This condition has 5DOF by allowing free motions in heave and pitch to account for the missing terms in the mathematical modeling due to the $3 \mathrm{DOF}$ assumption. The $\mathrm{FR}_{z \theta \phi}$ condition has complete $6 \mathrm{DOF}$ including free roll motion. Herein a few ratio variables are defined to facilitate the comparisons as $\xi_{z \theta} \equiv x_{z \theta} / x_{0}$ and $\xi_{\sigma \tau} \equiv x_{\sigma \tau} / x_{0}$, where $x_{0}, x_{\sigma \tau}$, and $x_{z \theta}$ are any variable $x$ using $\mathrm{FX}_{0}$, $\mathrm{FX}_{\sigma \tau}$, and $\mathrm{FR}_{z \theta}$, respectively, along with the variable $\zeta_{\phi}$ as defined in the previous section. The ratio $\xi_{z \theta}$ signifies the effects of the additional 2DOF (heave and pitch) of $\mathrm{FR}_{\mathrm{z} \theta}$ with respect to $\mathrm{FX}_{0}$ of $3 \mathrm{DOF}$. The ratio $\xi_{\sigma \tau}$ signifies the effects of $\sigma$ and $\tau$ relative to $\mathrm{FX}_{0}$ and can be interpreted 
as the effectiveness of $\mathrm{FX}_{\sigma \tau}$ in approximating $\mathrm{FR}_{\mathrm{z} \theta}$ by imposing a fixed $\sigma$ and $\tau$ instead of allowing free heave and pitch motions. Lastly, the ratio $\zeta_{\phi}$ signifies the effects of roll motion with respect to $\mathrm{FR}_{\mathrm{z} \theta}$.

Fig. 20(a)-(c) compares the static drift $X, Y$, and $N$ data for $F r=0.280$ among the different mount conditions. In the figure, data are curve-fitted to quadratic or cubic polynomial functions as discussed in Section 6.1. The $\mathrm{FR}_{\mathrm{z} \theta}$ data shown earlier in Fig. 8 are re-displayed using dashed-lines for comparisons. Data exhibit in general a clear distinction between the fixed- and free-mount cases. For $X$, the value at $\beta=0^{\circ}$ is mildly larger for $\mathrm{FR}_{\mathrm{z} \theta}$ than $\mathrm{FX}_{0}$ with $\xi_{\mathrm{z} \theta}=1.07$. However, the difference grows larger as $\beta$ increases, $\xi_{\mathrm{z} \theta}=1.14$ and 1.26 at $\beta=10^{\circ}$ and $20^{\circ}$, respectively. Similar trends are observed from $Y$ and $N$, with $\xi_{z \theta}=1.11$ and 1.24 for the former and 1.17 and 1.33 for the latter variable, at $\beta=10^{\circ}$ and $20^{\circ}$, respectively. The difference between $\mathrm{FX}_{0}$ and $\mathrm{FX}_{\sigma \tau}$ is relatively small with $\xi_{\sigma \tau}=1.03$, 1.05, and 1.07 for $X, Y$, and $N$, respectively, almost commonly at all $\beta$ values. The difference between $\mathrm{FR}_{\mathrm{z} \theta}$ and $\mathrm{FR}_{\mathrm{z} \theta \phi}$ is usually even smaller with $\zeta_{\phi}=1.00$ at $\beta=10^{\circ}$ and $\approx 0.96$ at $\beta=20^{\circ}$ for all variables.

Fig. 21(a)-(c) compares the time-histories of dynamic $X, Y$, and $N$ for pure sway $\left(\beta_{\max }=\right.$ $\left.10^{\circ}\right)$, pure yaw $\left(r_{\max }=0.3\right)$, and yaw-and-drift $\left(\beta=10^{\circ}\right)$ tests at $F r=0.280$, respectively, among the different mount conditions. The time-histories for $\mathrm{FR}_{\mathrm{z} \theta}$ shown earlier in Fig. 11 are redisplayed using dashed-lines for comparisons. For pure sway time-histories, the difference between $\mathrm{FX}_{0}$ and $\mathrm{FR}_{\mathrm{z} \theta}$ is relatively large with $\xi_{\mathrm{z} \theta}=1.34,1.12$, and 1.21 for $X_{0}, Y_{1}$, and $N_{1}$, respectively, where $X_{0}$ is the steady component of $X$ and $Y_{1}$ and $N_{1}$ the $1^{\text {st }}$-order harmonic amplitude of $Y$ and $N$ time-histories, respectively. The difference is relatively mild between $\mathrm{FX}_{0}$ and $\mathrm{FX}_{\sigma \tau}$ with $\xi_{\sigma \tau}=0.98,1.08$, and 1.09, respectively. For pure yaw time-histories, the differences among $\mathrm{FX}_{0}, \mathrm{FX}_{\sigma \tau}$, and $\mathrm{FR}_{\mathrm{z} \theta}$ are all mild with $\xi_{\mathrm{z} \theta}=1.10,1.00,1.13$ and $\xi_{\sigma \tau}=0.94,1.00,1.10$, for $X_{0}, Y_{1}$, $N_{1}$, respectively. For yaw-and-drift time-histories, the apparent difference between the fixed- and free-mount conditions is quite clear. Between $\mathrm{FX}_{0}$ and $\mathrm{FR}_{\mathrm{z} \theta}$, the difference of the static component is $\xi_{z \theta}=1.14,1.13$, and 1.22 for $X_{0}, Y_{0}$, and $N_{0}$, respectively, and the difference of the $1^{\text {st }}$ order harmonic amplitude is $\xi_{\mathrm{z} \theta}=3.74,1.13$, and 1.25 for $X_{1}, Y_{1}$, and $N_{1}$, respectively. Nonetheless, the difference between $\mathrm{FR}_{\mathrm{z} \theta}$ and $\mathrm{FR}_{\mathrm{z} \theta \phi}$ is rather small for all test types with typically $\zeta_{\phi}=$ $0.99 \sim 1.01$ for all variables. Also, for $Y$ and $N$ time-histories, the phase angle of the $1^{\text {st }}$-order 
harmonic is almost the same among different mount conditions with $\xi_{z \theta}, \xi_{\sigma \tau}, \zeta_{\phi}=0.98 \sim 1.03$ for all test types in general.

A common trend in the comparisons of both static and dynamic tests is that the difference is relatively large between the fixed- and free-mount conditions. This trend indicates that the forces and moment components in the horizontal plane are coupled with the motions in the vertical plane, particularly with heave and pitch motions. This coupled effect is demonstrated in Fig. 9(a) where the $\sigma$ and $\tau$ data in Fig. 9(b) are curve-fitted to $C_{T 15 \mathrm{C}}$ using a multivariate regression equation in the form $C_{T 15 \mathrm{C}}(\sigma, \tau)=A+B \sigma+C \tau+D \sigma \tau$, where $A, B, C$, and $D$ are the curve-fit coefficients. The relationships between $C_{T 15 \mathrm{C}}$ and $X$ and between $(\sigma, \tau)$ and $(z, \theta)$ are discussed in Sections 6.1 and 6.4, respectively. The regression result shows a strong correlation among the variables with a correlation coefficient $r=0.99$, except for the low $F r<0.1$ range where the friction resistance is dominant over the residual resistance. This coupling contradicts the 3DOF assumption of the mathematical modeling by Abkowitz (1964) and justifies the use of 5DOF in the model setup of standard PMM procedures to account for the effects of heave and pitch motions on hydrodynamic derivatives.

Table 15 presents hydrodynamic derivatives values associated with the $\mathrm{FX}_{0}, \mathrm{FX}_{\sigma \tau}$, and $\mathrm{FR}_{\mathrm{z} \theta \phi}$ mount conditions at $\mathrm{Fr}=0.280$. The evaluations of the sway-velocity derivatives (including $X_{*}$ ) are done using the static drift data for all mount condition cases. The evaluations of the other derivatives are done using the $\mathrm{MR}_{\mathrm{L}}$ method for the $\mathrm{FX}_{0}$ and $\mathrm{FR}_{\mathrm{z} \theta}$ cases and using the $\mathrm{SR}_{\mathrm{L}}$ method for the $\mathrm{FR}_{\mathrm{z} \theta \phi}$ case. The relative values to $\mathrm{FR}_{\mathrm{z} \theta}$ (presented in Table 11) of the linear derivatives $\left(X_{*}, Y_{v}, N_{v}, Y_{r}, N_{r}\right)$ in average are 0.98(0.08), 1.00(0.05), and 0.99(0.07) for $\mathrm{FX}_{0}, \mathrm{FX}_{\sigma \tau}$, and $\mathrm{FR}_{\mathrm{z} \theta \phi}$, respectively, where the values inside the parenthesis are the standard deviation from the average. Similarly, the relative values of the non-linear derivatives $\left(X_{v v}, Y_{v v v}, N_{v v v}, X_{r r}\right.$, $\left.Y_{r r r}, N_{r r r}, X_{v r}, Y_{v r r}, Y_{r v v}, N_{v r r}, N_{r v v}\right)$ are 0.53(0.26), 0.64(0.25), and 1.13(0.92), respectively, and those of the acceleration derivatives $\left(Y_{\dot{v}}, N_{\dot{v}}, Y_{\dot{r}}, N_{\dot{r}}\right)$ are $0.81(0.34), 0.67(0.19)$, and 0.96(0.19), respectively. Thus, the effect of using a fixed-mount condition (or the effect of heave and pitch motions) is less significant on the linear derivatives but can be considerable on the nonlinear derivatives or the acceleration derivatives. However, the effect of the roll motion is less significant on all derivatives, if the hull form of interest is similar as with the present study.

\section{CONCLUSIONS}


Towing-tank experiments are performed for a surface combatant advancing in calm water as it undergoes static and dynamic planar motion mechanism (PMM) maneuvers. The geometry is a $1 / 46.6$ scale DTMB model 5512 of with $L=3.048 \mathrm{~m}$, which is a geosym of DTMB model 5415. The experiments are performed in a $100 \mathrm{~m} \times 3.048 \mathrm{~m} \times 3.048 \mathrm{~m}$ towing tank. The measurement system is a custom-designed planar-motion-mechanism (PMM), a 6-component load cell, and a Krypton contactless motion tracker. Data includes static and dynamic maneuvering forces and moments and motions. The quality of data is assessed by evaluating statistical convergence and assessing measurement uncertainty following standard procedures. The forces and moment measurements and uncertainty assessments are in collaboration with two other international towing tank facilities (FORCE and INSEAN) and the $24^{\text {th }}-25^{\text {th }}$ ITTC Maneuvering Committee. The collaboration includes an overlapping PMM test matrix using the same model geometry with different scales for validation of procedures and identification of facility biases and scale effects.

Statistical convergence of the measurement variables is analyzed by monitoring the convergence of the confidence interval size of the variable values. Most of both the static and dynamic variables show a small convergence error $E_{\mathrm{SC}}(\%)<1$. The total uncertainty is assessed at about $3 \sim 8 \%$ for the static variables and about $5 \sim 8 \%$ for the dynamic variables. The asymmetry bias was large for $X$ and $\theta$ data commonly for static and dynamic tests. The evaluations of the rotary and acceleration derivatives are done by using either the MR method or the SR method. The difference between the derivatives values by the two methods is usually small for the linear derivatives; however, the difference is usually large for the non-linear derivatives. Based on reconstructions of the measured forces and moment time-histories, the MR method (particularly the $M R_{L}$ method) is assessed to give smaller reconstruction errors than the SR method. Thus, the $\mathrm{MR}_{\mathrm{L}}$ method is the most robust approach among other evaluation methods from the present study. For the use of the SR method, the use of sufficiently large $v_{\max }$ or $r_{\max }$ value is important if the non-linear derivatives are also desired from the evaluation. In general, linear derivatives are less sensitive to $F r$ change but the changes of non-linear derivatives with $F r$ are considerable. In addition, the linear and non-linear static derivatives and the non-linear rotary derivatives are independent of model size but the linear rotary derivatives and the acceleration derivatives exhibit correlations with model size. Particularly, the rotary acceleration derivatives show a rather strong correlation, i.e., their values increase largely as the model size increases. Regarding 
the hull motions, the coupling is weak between heave and roll but strong between pitch and roll. The effects of heave and pitch motions on the horizontal plane forces and moment components are considerable for both static and dynamic maneuvers. However, the effects of roll motions on the horizontal plane forces and moment are small for the present study.

The present work provides a readily accessible maneuvering data set with documentation of mathematical modeling and rigorous statistical convergence and uncertainty assessment of the measurement results. The results are also useful for CFD validation and are archived at http:// http://www.iihr.uiowa.edu/shiphydro. The limitation of the present work includes the use of an un-appended model except for bilge keels. Future work should include and address the effects by the presence of appendages and propellers.

\section{ACKNOWLEDGMENTS}

This research was sponsored by the Office of Naval Research under Grant N00014-01-10073 under the administration of Dr. Ki-Han Kim, whose support is greatly appreciated. Special thanks are also extended to University of Iowa mechanical and civil engineering undergraduates (Evan Aschbrenner, Peter Carusona, Mike Elgin, Nathan Hicks, Matt Marquardt, Ryan Nielsen, Rob White, Issac Johnson, Scott Salsbery, and Chelsea Cross) who assisted in the data acquisition phase of this study.

\section{REFERENCES}

Abkowitz, M. A., 1964, “Lectures on Ship hydrodynamics - Steering and maneuvering," Hydroand Aerodynamics Laboratory Report Hy-5, Lyngby, Denmark.

AGARD AR-304, 1994, "Quality Assessment for Wind Tunnel Testing," Advisory Group for Aerospace Research and Development, North Atlantic Treaty Organization, Neuilly-surSeine, France.

AIAA S-071A-1999, "Assessment of Experimental Uncertainty with Application to Wind Tunnel Testing," American Institute of Aeronautics and Astronautics, Reston, Virginia, USA.

Araki, M., Sadat-Hosseini, H., Sanada, Y., Umeda, N., and Stern, F., 2012, "Estimating maneuvering coefficients using system identification methods with experimental, systems-based, and CFD free running trial data," Ocean Engineering, Volume 51, pp. 63-84. 
ASME PTC 19-1-1998, "Test Uncertainty," American Society of Mechanical Engineers, New York, USA.

ASME PTC 19-1-2005, “Test Uncertainty," American Society of Mechanical Engineers, New York, USA.

Bendat, J. S. and Piersol, A. G., 1966, Measurement and Analysis of Random Data, John Wiley and Sons, Inc., New York, USA.

Bennedetti, L., Bouscasse, B., Broglia, R., Fabbri, L., La Gala, F., and Gugni, C., 2006, "PMM Model Test with DDG51 Including Uncertainty Assessment," INSEAN Report No. 14.

Bishop, R. E. D. \& Parkinson, A. G., 1970, “On the planar motion mechanism used in ship model testing," Philosophical Transactions for Royal Society of London, Series A, Mathematical and Physical Sciences, Vol. 266, pp. 35-61.

Bhushan, S., Xing, T., Carrica, P., and Stern, F., 2009, "Model- and Full-scale URANS Simulations of Athena Resistance, Powering, Seakeeping, and 5415 Maneuvering," Journal of Ship Research, Vol. 53, pp. 1-21.

Bhushan, S., Carrica, P., Yang, J., and Stern, F., 2011, "Scalability studies and large grid computations for surface combatant using CFDShip-Iowa," International Journal of High Performance Computing Applications, Vol. 25, No. 4, pp. 466-487.

Carrica, P., Ismail, F., Hyman, M., Bhushan, S., and Stern, F., 2013, “Turn and Zigzag Maneuvers of a Surface Combatant Using a URANS Approach with Dynamic Overset Grids," Journal of Marine Science and Technology, Vol. 18, No. 2, pp. 166-181.

Chislett, M. S., 1990, "The addition of heel-roll servo mechanism to the DMI horizontal Planar Motion Mechanism,” Proc. of MARSIM and ICSM '90, Tokyo, Japan.

Cura Hochbaum, A., 2006, "Virtual PMM Tests for Manoeuvring Prediction," the $26^{\text {th }}$ ONR Symposium on Naval Hydrodynamics, Rome, Italy. 
Di Mascio, A., Broglia, R., and Muscari, R., 2007, "Numerical Simulations of Viscous Flow Around a Fully Appended Hull with Enforced Motion," the $9^{\text {th }}$ Numerical Ship Hydrodynamics Conference, Vol. I, pp. 191-207.

Dubbiosso, G., Durante, D., Broglia, R., and Di Mascio, A., 2012, "CFD Maneuvering Prediction of a Twin Screw Vessel with Different Stern Appendages Configuration," the $29^{\text {th }}$ Symposium on Naval Hydrodynamics, Gothenburg, Sweden.

Fossen, T. I., 1994, Guidance and Control of Ocean Vehicles, John Wiley \& Sons Ltd., England.

Gertler, M., 1959, "Symposium on Towing Tank Facilities," Instrument and Measuring Technique, Zagreb, Paper 6.

Goodman, A., "Experimental Techniques and Methods of Analysis Used in Submerged Body Research," Proc. $3^{\text {rd }}$ Symposium on Naval Hydrodynamics, Scheveningen 1960.

Hosseini, H., Carrica, P., Stern, F., Umeda, N., Hashimoto, H., Matsuda, A., 2011, “CFD, System-Based and EFD Study of Ship Dynamic Instability Events: Surf-riding, Periodic Motion, and Broaching,” Ocean Engineering, Vol. 38, Issue 1, pp. 88-110.

Hyman, M. C., Moraga, F. J., Drew, D. A., and Lahey, R. T., 2006, "Computation of the unsteady two-phase flow around a maneuvering surface ship," the $26^{\text {th }}$ ONR Symposium on Naval Hydrodynamics, Vol. 2, pp. 39-58.

International Maritime Organization (IMO, 2002): Resolution "Standards for Ship Maneuverability," MSC.137(76) 4 December 2002.

Irvine, M., Longo, J., and Stern F., 2008, "Pitch and Heave Tests and Uncertainty Assessment for a Surface Combatant in Regular Head Waves," Journal of Ship Research, Vol. 52, No. 2, pp. 146-163.

Kreyszig, E., 1998, Advanced Engineering Mathematics, Wiley.

Larsson, L., Stern, F., Visonneau, M., 2013, "CFD in Ship Hydrodynamics: Results of the Gothenburg 2010 Workshop,” IV International Conference Marine 2011 International Confe- 
rence Computational Methods Marine Engineering: Selected Papers, Editor Eca et al., Springer, pp. 237-260.

Lewandowski, E. M., 2004, The Dynamics of Marine Craft: Maneuvering and Seakeeping, World Scientific Publishing Co. Pte. Ltd. Singapore.

Longo, J. and Stern, F., 2002, "Effects of Drift Angle on Model-Scale Ship Flow," Experiments in Fluids, Vol. 32, pp. 558-569.

Longo, J., and Stern, F., 2005, "Uncertainty Assessment for Towing Tank Tests With Example for Surface Combatant DTMB Model 5415,” J. of Ship Research, Vol. 49, No. 01, pp. 55-68.

Sadat-Hosseini, H., Stern F., Toxopeus S., Visonneau M., Guilmineau E., Lin W.M., and Grigoropoulos G., 2014, “CFD, Potential Flow, and System-based Simulations Of Course Keeping in Calm Water and Seakeeping in Regular Waves for 5415M”, In preparation for Journal of Ocean Engineering.

Sakamoto, N., Carrica, P., and Stern, F., 2012, "URANS Simulations of Static and Dynamic Maneuvering for Surface Combatant: Part 1. Verification and Validation for Forces, Moment, and Hydrodynamic Derivatives," Journal of Marine Science and Technology, Vol. 17, No. 4, pp. $422-445$.

Sakamoto, N., Carrica, P., and Stern, F., 2012, "URANS Simulations of Static and Dynamic Maneuvering for Surface Combatant: Part 2. Analysis and Validation for Local Flow Characteristics,” Journal of Marine Science and Technology, Vol. 17, No. 4, pp. 446-468.

Simonsen, C. D., 2004, "PMM Model Test with DDG51 Including Uncertainty Assessment," Force Technology Report No. ONRII187 01, 145 pp.

Simonsen, C. D., and Stern, F., 2005, "RANS Maneuvering Simulation of Esso Osaka with Rudder and a Body-Force Propeller,” Journal of Ship Research, Vol. 49, No. 2, pp. 98-120.

Simonsen, C. D., Otzen, J., Klimt, C., Larsen, N. L., and Stern, F., 2012, "Maneuvering Predictions in the Early Design Phase Using CFD Generated PMM Data," the $29^{\text {th }}$ Symposium on Naval Hydrodynamics, Gothenburg, Sweden. 
Stern, F., Olivieri, A., Shao, J., Longo, J., and Ratcliffe, T., 2005, "Statistical Approach for Estimating Intervals of Certification or Biases of Facilities or Measurement System Including Uncertainties,” ASME J. Fluids Eng., Vol. 127, No. 2, pp. 604-610.

Stern, F., Agdrup, K., Kim, S.Y., Hochbaum, A. C., Rhee, K. P., Quadvlieg, F., Perdon, P., Hino, T., Broglia, R., and Gorski, J., 2011, "Experience from SIMMAN 2008: The First Workshop On Verification And Validation Of Ship Maneuvering Simulation Methods," Journal of Ship Research, Volume 55, Number 2, pp. 135-147.

Strøm-Tejsen, J. and Chislett, M. S., 1966, “A Model Testing Technique and Method of Analysis for the Prediction of Steering and Manoeuvring Qualities of Surface Vessels," Hydro \& Aerodynamic Laboratory Report No. Hy-7, Lyngby, Denmark.

Tahara, Y., Longo, J., Stern, F., and Himeno, Y., 2002, "Comparison of CFD and EFD for Series $60 \mathrm{CB}=.6$ in Steady Drift Motion," Journal of Marine Science and Technology, Vol. 7, No. 1, pp. 17-30.

Williams, C. A., Jr., 1950, "On the Choice of the Number and Width of Classes for Chi-Square Test of Goodness of Fit," J. Am. Statistical Assoc., 45, pp. 77-86.

Xing, T., Bhushan, S., and Stern, F., 2012, "Vortical and turbulent structures for KVLCC2 at drift angle 0,12, and 30 degrees," Ocean Engineering, Vol. 55, pp. 23-43. 
Table 1 Mathematical models for dynamic PMM (Harmonics in equation (8)).

\begin{tabular}{|c|c|c|}
\hline$X$-harmonics & $Y$-harmonics & $N$-harmonics \\
\hline \multicolumn{3}{|c|}{ Pure sway $\left(v=-v_{\max } \cos \omega t ; \dot{v}=\dot{v}_{\max } \sin \omega t\right)$} \\
\hline $\begin{array}{l}X_{0}=X_{*}+\frac{1}{2} X_{v v} v_{\max }^{2} \\
X_{\mathrm{C} 1}=X_{S 1}=0 \\
X_{\mathrm{C} 2}=\frac{1}{2} X_{v v} v_{\max }^{2} \\
X_{\mathrm{S} 2}=0 \\
X_{\mathrm{C} 3}=X_{\mathrm{S} 3}=0\end{array}$ & $\begin{array}{l}Y_{0}=0 \\
Y_{\mathrm{C} 1}=-\left(Y_{v} v_{\max }+\frac{3}{4} Y_{v v v} v_{\text {max }}^{3}\right) \\
Y_{\mathrm{S} 1}=Y_{\dot{v}} \dot{v}_{\text {max }} \\
Y_{\mathrm{C} 2}=Y_{\mathrm{S} 2}=0 \\
Y_{\mathrm{C} 3}=-\frac{1}{4} Y_{v v v} v_{\text {max }}^{3} \\
Y_{\mathrm{S} 3}=0\end{array}$ & $\begin{array}{l}N_{0}=0 \\
N_{\mathrm{C} 1}=-\left(N_{v} v_{\max }+{ }^{3} N_{v v v} v_{\text {max }}^{3}\right) \\
N_{\mathrm{S} 1}=N_{\dot{v}} \dot{v}_{\max } \\
N_{\mathrm{C} 2}=N_{\mathrm{S} 2}=0 \\
N_{\mathrm{C} 3}=-\frac{1}{4} N_{v v v} v_{\max }^{3} \\
N_{\mathrm{S} 3}=0\end{array}$ \\
\hline \multicolumn{3}{|c|}{ Pure yaw $\left(r=r_{\max } \sin \omega t ; \dot{r}=\dot{r}_{\max } \cos \omega t\right)$} \\
\hline $\begin{array}{l}X_{0}=X_{*}+\frac{1}{2} X_{r r} r_{\max }^{2} \\
X_{\mathrm{C} 1}=X_{\mathrm{S} 1}=0 \\
X_{\mathrm{C} 2}=-\frac{1}{2} X_{r r} r_{\max }^{2} \\
X_{\mathrm{S} 2}=0 \\
X_{\mathrm{C} 3}=X_{\mathrm{S} 3}=0\end{array}$ & $\begin{array}{l}Y_{0}=0 \\
Y_{\mathrm{C} 1}=Y_{\dot{r}} \dot{r}_{\max } \\
Y_{\mathrm{S} 1}=Y_{r} r_{\max }+\frac{3}{4} Y_{r r r} r_{\max }^{3} \\
Y_{\mathrm{C} 2}=Y_{\mathrm{S} 2}=0 \\
Y_{\mathrm{C} 3}=0 \\
Y_{\mathrm{S} 3}=-\frac{1}{4} Y_{r r r} r_{\max }^{3}\end{array}$ & $\begin{array}{l}N_{0}=0 \\
N_{\mathrm{C} 1}=N_{\dot{r}} \dot{r}_{\max } \\
N_{\mathrm{S} 1}=N_{r} r_{\max }+\frac{3}{4} N_{r r r} r_{\max }^{3} \\
N_{\mathrm{C} 2}=N_{\mathrm{S} 2}=0 \\
N_{\mathrm{C} 3}=0 \\
N_{\mathrm{S} 3}=-\frac{1}{4} N_{r r r} r_{\max }^{3}\end{array}$ \\
\hline \multicolumn{3}{|c|}{$\underline{\text { Yaw-and-drift }\left(r=r_{\max } \sin \omega t ; \dot{r}=\dot{r}_{\max } \cos \omega t ; v=\text { constant }\right)}$} \\
\hline $\begin{array}{l}X_{0}=X_{*}+X_{v v} v^{2}+\frac{1}{2} X_{r r} r_{\max }^{2} \\
X_{\mathrm{C} 1}=0 \\
X_{\mathrm{S} 1}=X_{v r} v r_{\max } \\
X_{\mathrm{C} 2}=-\frac{1}{2} X_{r r} r_{\max }^{2} \\
X_{\mathrm{S} 2}=0 \\
X_{\mathrm{C} 3}=X_{\mathrm{S} 3}=0\end{array}$ & $\begin{aligned} Y_{0} & =Y_{v} v+Y_{v v v} v^{3}+\frac{1}{2} Y_{v r r} v r_{\max }^{2} \\
Y_{\mathrm{C} 1} & =Y_{\dot{r}} \dot{r}_{\max } \quad \begin{array}{l}3 \\
Y_{\mathrm{S} 1}\end{array}=Y_{r} r_{\max }+\frac{3}{4} Y_{r r r} r_{\max }^{3} \\
\quad & Y_{r v v} r_{\max } v^{2} \\
Y_{\mathrm{C} 2} & =-\frac{1}{2} Y_{v r r} v r_{\max }^{2} \\
Y_{\mathrm{S} 2} & =0 \\
Y_{\mathrm{C} 3} & =0 \\
Y_{\mathrm{S} 3} & =-\frac{1}{4} Y_{r r r} r_{\max }^{3}\end{aligned}$ & 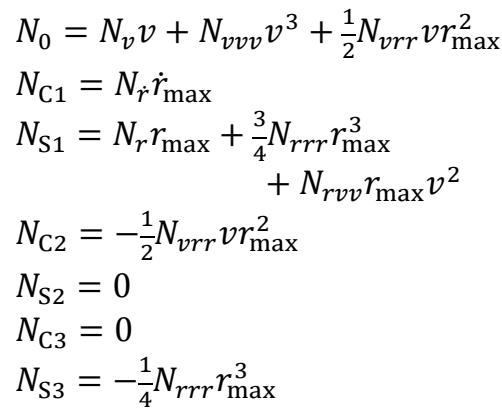 \\
\hline
\end{tabular}


Table 2 Multiple-Run (MR) method.

\begin{tabular}{|c|c|c|c|}
\hline Test & Harmonics Curve-fit Functions & \multicolumn{2}{|l|}{ Hydrodynamic Derivatives } \\
\hline \multicolumn{4}{|c|}{$M R_{L}$ Method } \\
\hline Pure sway & $\begin{array}{l}X_{0}\left(v_{\max }\right)=A+B v_{\max }^{2} \\
Y_{\mathrm{C} 1}, N_{\mathrm{C} 1}\left(v_{\max }\right)=C v_{\max }+D v_{\max }^{3} \\
Y_{\mathrm{S} 1}, N_{\mathrm{S} 1}\left(\dot{v}_{\max }\right)=E \dot{v}_{\max }\end{array}$ & $\begin{array}{l}X_{*}=A \\
Y_{v}, N_{v}=-C \\
Y_{\dot{v}}, N_{\dot{v}}=E\end{array}$ & $\begin{array}{l}X_{v v}=2 B \\
Y_{v v v}, N_{v v v}=-4 D / 3\end{array}$ \\
\hline Pure yaw & $\begin{array}{l}X_{0}\left(r_{\max }\right)=A+B r_{\max }^{2} \\
Y_{\mathrm{S} 1}, N_{\mathrm{S} 1}\left(r_{\max }\right)=C r_{\max }+D r_{\max }^{3} \\
Y_{\mathrm{C} 1}, N_{\mathrm{C} 1}\left(\dot{r}_{\max }\right)=E \dot{r}_{\max }\end{array}$ & $\begin{array}{l}X_{*}=A \\
Y_{r}, N_{r}=C \\
Y_{\dot{r}}, N_{\dot{r}}=E\end{array}$ & $\begin{array}{l}X_{r r}=2 B \\
Y_{r r r}, N_{r r r}=4 D / 3\end{array}$ \\
\hline Yaw-and-drift & $\begin{array}{l}X_{\mathrm{C} 1}(v)=A v \\
Y_{0}, N_{0}(v)=B v+C v^{3} \\
Y_{\mathrm{S} 1}, N_{\mathrm{S} 1}(v)=D+E v^{2}\end{array}$ & $\begin{array}{l}X_{v r}=A / r_{\max } \\
Y_{v r r}=2\left(B-Y_{v}\right) / r_{\max }^{2} \\
Y_{r v v}, N_{r v v}=E / r_{\max }\end{array}$ & $N_{v r r}=2\left(B-N_{v}\right) / r_{\max }^{2}$ \\
\hline \multicolumn{4}{|c|}{$M R_{H}$ Method } \\
\hline Pure sway & $\begin{array}{l}X_{\mathrm{C} 2}\left(v_{\max }\right)=A v_{\max }^{2} \\
Y_{\mathrm{C} 3}, N_{\mathrm{C} 3}\left(v_{\max }\right)=B v_{\max }^{3}\end{array}$ & $\begin{array}{l}X_{v v}=2 A \\
Y_{v v v}, N_{v v v}=-4 B\end{array}$ & \\
\hline Pure yaw & $\begin{array}{l}X_{\mathrm{C} 2}\left(r_{\max }\right)=A r_{\text {max }}^{2} \\
Y_{\mathrm{S} 3}, N_{\mathrm{S} 3}\left(r_{\text {max }}\right)=B r_{\max }^{3}\end{array}$ & $\begin{array}{l}X_{r r}=-2 A \\
Y_{r r r}, N_{r r r}=-4 B\end{array}$ & \\
\hline Yaw-and-drift & $Y_{\mathrm{C} 2}, N_{\mathrm{C} 2}(v)=A v$ & $Y_{v r r}, N_{v r r}=-2 A / r_{\max }^{2}$ & \\
\hline
\end{tabular}

Table 3 Single-Run (SR) method.

\begin{tabular}{llll}
\hline Method & Pure sway & Pure yaw & Yaw-and-drift \\
\hline $\mathrm{SR}_{\mathrm{L}}$ & $X_{*}=X_{0}-X_{2}$ & $X_{*}=X_{0}+X_{\mathrm{C} 2}$ & $X_{v r}=\frac{1}{v r_{\max }} X_{\mathrm{S} 1}$ \\
$X_{v v}=\frac{2}{v_{\max }^{2}} X_{\mathrm{C} 2}$ & $X_{r r}=-\frac{2}{r_{\max }^{2}} X_{\mathrm{C} 2}$ & $Y_{v r r}=\frac{2}{v r_{\max }^{2}}\left(Y_{0}-Y_{v} v-Y_{v v v} v^{3}\right)$ \\
$Y_{v}=-\frac{1}{v_{\max }}\left(Y_{\mathrm{C} 1}-3 Y_{\mathrm{C} 3}\right)$ & $Y_{r}=\frac{1}{r_{\max }}\left(Y_{\mathrm{S} 1}+3 Y_{\mathrm{S} 3}\right)$ & $Y_{r v v}=\frac{1}{r_{\max } v^{2}}\left(Y_{\mathrm{S} 1}-Y_{r} r_{\max }-\frac{3}{4} Y_{r r r} r_{\max }^{3}\right)$ \\
$Y_{v v v}=-\frac{4}{v_{\max }^{3}} Y_{\mathrm{C} 3}$ & $Y_{r r r}=-\frac{4}{r_{\max }} Y_{\mathrm{S} 3}$ & $N_{v r r}=\frac{2}{v r_{\max }^{2}}\left(N_{0}-N_{v} v-N_{v v v} v^{3}\right)$ \\
$Y_{\dot{v}}=\frac{1}{\dot{v}_{\max }} Y_{\mathrm{S} 1}$ & $Y_{\dot{r}}=\frac{1}{\dot{r}_{\max }} Y_{\mathrm{C} 1}$ & $N_{r v v}=\frac{1}{r_{\max } v^{2}}\left(N_{\mathrm{S} 1}-N_{r} r_{\max }-\frac{3}{4} N_{r r r} r_{\max }^{3}\right)$ \\
$N_{v}=-\frac{1}{v_{\max }}\left(N_{\mathrm{C} 1}-3 N_{\mathrm{C} 3}\right)$ & $N_{r}=\frac{1}{r_{\max }}\left(N_{\mathrm{S} 1}+3 N_{\mathrm{S} 3}\right)$ & \\
$N_{v v v}=-\frac{4}{v_{\max }^{3}} N_{\mathrm{C} 3}$ & $N_{r r r}=-\frac{4}{r_{\max }^{3}} N_{\mathrm{S} 3}$ & \\
$N_{\dot{v}}=\frac{1}{\dot{v}_{\max }} N_{\mathrm{S} 1}$ & $N_{\dot{r}}=\frac{1}{\dot{r}_{\max }} N_{\mathrm{C} 1}$ & \\
\hline $\mathrm{SR}_{\mathrm{H}}$ & & $Y_{v r r}=-\frac{2}{v r_{\max }^{2}} Y_{\mathrm{C} 2}$ \\
& & $N_{v r r}=-\frac{2}{v r_{\max }^{2}} N_{\mathrm{C} 2}$ \\
\hline
\end{tabular}


Table 4 Particular solutions of heave, pitch, and roll motion equations for PMM.

\begin{tabular}{|c|c|c|}
\hline Test & $z_{\mathrm{p}}, \theta_{\mathrm{p}}($ with $\phi=0)$ & $\phi_{\mathrm{p}}$ \\
\hline \multirow[t]{4}{*}{ Pure sway } & $\left(z_{0}, \theta_{0}\right)^{\mathrm{T}}=\mathbf{A}+\mathbf{B} v_{\max }^{2}$ & $\phi_{0}=0$ \\
\hline & $\left(z_{\mathrm{C} 1}, z_{\mathrm{S} 1}, \theta_{\mathrm{C} 1}, \theta_{\mathrm{S} 1}\right)^{\mathrm{T}}=\mathbf{0}$ & $\left(\phi_{\mathrm{C} 1}, \phi_{\mathrm{S} 1}\right)^{\mathrm{T}}=\mathbf{A} \dot{v}_{\max }+\mathbf{B} v_{\max }+\mathbf{C} v_{\max }^{3}$ \\
\hline & $\left(z_{\mathrm{C} 2}, z_{\mathrm{S} 2}, \theta_{\mathrm{C} 2}, \theta_{\mathrm{S} 2}\right)^{\mathrm{T}}=\mathbf{C} v_{\max }^{2}$ & $\left(\phi_{\mathrm{C} 2}, \phi_{\mathrm{S} 2}\right)^{\mathrm{T}}=\mathbf{0}$ \\
\hline & $\left(z_{\mathrm{C} 3}, z_{\mathrm{S} 3}, \theta_{\mathrm{C} 3}, \theta_{\mathrm{S} 3}\right)^{\mathrm{T}}=\mathbf{0}$ & $\left(\phi_{\mathrm{C} 3}, \phi_{\mathrm{S} 3}\right)^{\mathrm{T}}=\mathbf{D} v_{\max }^{3}$ \\
\hline \multirow[t]{4}{*}{ Pure yaw } & $\left(z_{0}, \theta_{0}\right)^{\mathrm{T}}=\mathbf{A}+\mathbf{B} r_{\max }^{2}$ & $\phi_{0}=0$ \\
\hline & $\left(z_{\mathrm{C} 1}, z_{\mathrm{S} 1}, \theta_{\mathrm{C} 1}, \theta_{\mathrm{S} 1}\right)^{\mathrm{T}}=\mathbf{0}$ & $\left(\phi_{\mathrm{C} 1}, \phi_{\mathrm{S} 1}\right)^{\mathrm{T}}=\mathbf{A} \dot{r}_{\max }+\mathbf{B} r_{\max }+\mathbf{C} r_{\max }^{3}$ \\
\hline & $\left(z_{\mathrm{C} 2}, z_{\mathrm{S} 2}, \theta_{\mathrm{C} 2}, \theta_{\mathrm{S} 2}\right)^{\mathrm{T}}=\mathbf{C} r_{\max }^{2}$ & $\left(\phi_{\mathrm{C} 2}, \phi_{\mathrm{S} 2}\right)^{\mathrm{T}}=\mathbf{0}$ \\
\hline & $\left(z_{\mathrm{C} 3}, z_{\mathrm{S} 3}, \theta_{\mathrm{C} 3}, \theta_{\mathrm{S} 3}\right)^{\mathrm{T}}=\mathbf{0}$ & $\left(\phi_{\mathrm{C} 3}, \phi_{\mathrm{S} 3}\right)^{\mathrm{T}}=\mathbf{D} r_{\max }^{3}$ \\
\hline \multirow[t]{4}{*}{ Yaw and drift } & $\left(z_{0}, \theta_{0}\right)^{\mathrm{T}}=\mathbf{A}+\mathbf{B} r_{\max }^{2}+\mathbf{C} v^{2}$ & $\phi_{0}=A v+B r_{\max }^{2} v+C v^{3}$ \\
\hline & $\left(z_{\mathrm{C} 1}, z_{\mathrm{S} 1}, \theta_{\mathrm{C} 1}, \theta_{\mathrm{S} 1}\right)^{\mathrm{T}}=\mathbf{D} r_{\max } v$ & $\left(\phi_{\mathrm{C} 1}, \phi_{\mathrm{S} 1}\right)^{\mathrm{T}}=\mathbf{A} \dot{r}_{\max }+\mathbf{B} r_{\max }+\mathbf{C} r_{\max }^{3}+\mathbf{D} r_{\max } v^{2}$ \\
\hline & $\left(z_{\mathrm{C} 2}, z_{\mathrm{S} 2}, \theta_{\mathrm{C} 2}, \theta_{\mathrm{S} 2}\right)^{\mathrm{T}}=\mathbf{E} r_{\max }^{2}$ & $\left(\phi_{\mathrm{C} 2}, \phi_{\mathrm{S} 2}\right)^{\mathrm{T}}=\mathbf{E} r_{\max }^{2} v$ \\
\hline & $\left(z_{\mathrm{C} 3}, z_{\mathrm{S} 3}, \theta_{\mathrm{C} 3}, \theta_{\mathrm{S} 3}\right)^{\mathrm{T}}=\mathbf{0}$ & $\left(\phi_{\mathrm{C} 3}, \phi_{\mathrm{S} 3}\right)^{\mathrm{T}}=\mathbf{F} r_{\max }^{3}$ \\
\hline
\end{tabular}

Table 5 Full- and model-scale particulars.

\begin{tabular}{cccccc}
\hline Item & \multirow{2}{*}{ Unit } & Full-scale & \multicolumn{3}{c}{ Model-scale } \\
\cline { 4 - 6 } & & & IIHR (A) & FORCE (B) & INSEAN (C) \\
\hline Scale & - & 1 & 46.588 & 35.48 & 24.83 \\
$L\left(L_{P P}\right)$ & $\mathrm{m}$ & 142.0 & 3.048 & 4.0023 & 5.72 \\
$L_{W L}$ & $\mathrm{~m}$ & 142.18 & 3.052 & 4.0083 & 5.7273 \\
$B_{W L}$ & $\mathrm{~m}$ & 19.10 & 0.410 & 0.5382 & 0.769 \\
$T\left(T_{m}\right)$ & $\mathrm{m}$ & 6.16 & 0.132 & 0.1736 & 0.248 \\
$\nabla$ & $\mathrm{m}^{3}$ & 8,472 & 0.086 & 0.1897 & 0.554 \\
$\Delta$ & $\mathrm{Ton}$ & 8,684 & 0.086 & 0.1897 & 0.554 \\
$C_{b}$ & - & 0.506 & 0.506 & 0.506 & 0.506 \\
$m$ & $\mathrm{Kg}$ & $\mathrm{n} / \mathrm{a}$ & $82.55(83.35)$ & 190.7 & 554.0 \\
$x_{G}$ & $\mathrm{~m}$ & $\mathrm{n} / \mathrm{a}$ & -0.0157 & $\mathrm{n} / \mathrm{a}$ & $\mathrm{n} / \mathrm{a}$ \\
$y_{G}$ & $\mathrm{~m}$ & $\mathrm{n} / \mathrm{a}$ & 0.0 & $\mathrm{n} / \mathrm{a}$ & $\mathrm{n} / \mathrm{a}$ \\
$z_{G}$ & $\mathrm{~m}$ & $\mathrm{n} / \mathrm{a}$ & 0.084 & $\mathrm{n} / \mathrm{a}$ & $\mathrm{n} / \mathrm{a}$ \\
$I_{x}$ & $\mathrm{Kg} \cdot \mathrm{m}^{2}$ & $\mathrm{n} / \mathrm{a}$ & 1.98 & $\mathrm{n} / \mathrm{a}$ & $\mathrm{n} / \mathrm{a}$ \\
$I_{y}$ & $\mathrm{Kg} \cdot \mathrm{m}^{2}$ & $\mathrm{n} / \mathrm{a}$ & 53.88 & $\mathrm{n} / \mathrm{a}$ & $\mathrm{n} / \mathrm{a}$ \\
$I_{z}$ & $\mathrm{Kg} \cdot \mathrm{m}^{2}$ & $\mathrm{n} / \mathrm{a}$ & $49.99(44.35)$ & 225.3 & 1151.4 \\
\hline
\end{tabular}

( ): Fixed-mount 
Table 6 Test conditions for IIHR PMM tests.

\begin{tabular}{|c|c|c|c|c|c|c|c|c|c|c|c|c|c|c|}
\hline \multicolumn{15}{|c|}{$\underline{\text { Static drift }}$} \\
\hline \multirow{4}{*}{$\begin{array}{c}F r \\
(-)\end{array}$} & \multirow{2}{*}{$\begin{array}{c}\begin{array}{c}U_{\mathrm{C}} \\
(\mathrm{m} / \mathrm{s})\end{array} \\
0.754\end{array}$} & \multicolumn{9}{|c|}{$\begin{array}{l}\beta \\
\left({ }^{\circ}\right)\end{array}$} & \multicolumn{4}{|c|}{$\underline{\text { Mount condition }}$} \\
\hline & & \multicolumn{9}{|c|}{$-20,-16,-12,-11,-\mathbf{- 1 0}^{1)}, 9,6,2,0,2,6,9, \mathbf{1 0}^{2)}, 11,12,16,20$} & \multirow{4}{*}{$\begin{array}{l}\mathbf{F X}_{\mathbf{0}} \\
\mathbf{F X}_{\mathbf{0}} \\
\mathbf{F X}_{\mathbf{0}}\end{array}$} & \multirow{3}{*}{$\mathbf{F X}_{\sigma \tau}$} & \multirow{3}{*}{\multicolumn{2}{|c|}{$\begin{array}{ll}F_{R_{\mathbf{\theta}}} & \mathbf{F R}_{\mathbf{z} \theta \phi} \\
\mathbf{F R}_{\mathbf{z} \theta} & \mathbf{F R}_{\mathbf{z} \theta \phi} \\
\mathbf{F R}_{\mathbf{z} \theta} & \mathrm{FR}_{\mathbf{z} \theta \phi}\end{array}$}} \\
\hline & 1.531 & & & $-16,-12$ & $-11,-\mathbf{1 0})^{1)}$, & $6,2,0$, & $6,9, \mathbf{1 0}$ & $1,12,1$ & & & & & & \\
\hline & 2.241 & & & -12 & $11,-\mathbf{1 0}^{1)}$ & $5,2,0,2$ & $, 9, \mathbf{1 0}^{2)}$ & 1,12 & & & & & & \\
\hline \multicolumn{14}{|c|}{ Pure sway } & \\
\hline $\begin{array}{l}F r \\
(-)\end{array}$ & $\begin{array}{c}U_{\mathrm{C}} \\
(\mathrm{m} / \mathrm{s})\end{array}$ & $\begin{array}{c}y_{\max } \\
(\mathrm{m})\end{array}$ & & & $\begin{array}{c}\beta_{\max } \\
\left({ }^{\circ}\right)\end{array}$ & $\begin{array}{c}v_{\max } \\
(-)\end{array}$ & $\begin{array}{c}\dot{v}_{\max } \\
(-)\end{array}$ & $\begin{array}{l}\omega_{1}^{\prime} \\
(-)\end{array}$ & $\begin{array}{l}\omega_{2}^{\prime} \\
(-)\end{array}$ & $\begin{array}{l}\omega_{3}^{\prime} \\
(-)\end{array}$ & \multicolumn{4}{|c|}{$\underline{\text { Mount condition }}$} \\
\hline \multirow[t]{3}{*}{0.280} & \multirow[t]{3}{*}{1.531} & 0.064 & \multicolumn{2}{|c|}{0.134} & 2 & 0.035 & 0.058 & 1.676 & 0.469 & 0.131 & $\mathrm{FX}_{0}$ & \multirow{3}{*}{$\begin{array}{l}\mathrm{FX}_{\sigma \tau} \\
\mathrm{FX}_{\sigma \tau} \\
\mathbf{F X}_{\sigma \tau}\end{array}$} & \multirow{3}{*}{$\begin{array}{l}\mathrm{FR}_{z \theta} \\
\mathrm{FR}_{\mathrm{z \theta}} \\
\mathbf{F R}_{\mathbf{z} \theta}\end{array}$} & \\
\hline & & 0.127 & & & 4 & 0.070 & 0.117 & 1.676 & 0.469 & 0.131 & $\mathrm{FX}_{0}$ & & & \\
\hline & & 0.317 & & & 10 & 0.174 & 0.291 & 1.676 & 0.469 & 0.131 & $\mathbf{F X}_{\mathbf{0}}$ & & & $\mathbf{F R}_{z \theta \phi}$ \\
\hline \multicolumn{15}{|c|}{ Pure vaw } \\
\hline $\begin{array}{l}F r \\
(-)\end{array}$ & $\begin{array}{c}U_{\mathrm{C}} \\
(\mathrm{m} / \mathrm{s})\end{array}$ & $\begin{array}{c}y_{\max } \\
(\mathrm{m})\end{array}$ & & & $\begin{array}{c}f \\
(\mathrm{~Hz})\end{array}$ & $\begin{array}{c}r_{\max } \\
(-)\end{array}$ & $\begin{array}{c}\dot{r}_{\max } \\
(-)\end{array}$ & $\begin{array}{l}\omega_{1}^{\prime} \\
(-)\end{array}$ & $\begin{array}{l}\omega_{2}^{\prime} \\
(-)\end{array}$ & $\begin{array}{l}\omega_{3}^{\prime} \\
(-)\end{array}$ & \multicolumn{4}{|c|}{ Mount condition } \\
\hline \multirow[t]{6}{*}{0.138} & \multirow[t]{6}{*}{0.754} & 0.041 & & & 0.076 & 0.05 & 0.10 & 1.930 & 0.266 & 0.037 & \multirow{6}{*}{$\begin{array}{l}\mathrm{FX}_{0} \\
\mathrm{FX}_{0} \\
\mathrm{FX}_{\mathbf{0}} \\
\mathrm{FX}_{0} \\
\mathrm{FX}_{0} \\
\mathrm{FX}_{0}\end{array}$} & & \multirow{6}{*}{$\begin{array}{l}\mathrm{FR}_{z \theta} \\
\mathrm{FR}_{z \theta} \\
\mathbf{F R}_{z \theta} \\
\mathrm{FR}_{z \theta} \\
\mathrm{FR}_{z \theta} \\
\mathrm{FR}_{z \theta}\end{array}$} & \\
\hline & & 0.122 & & & 0.076 & 0.15 & 0.29 & 1.930 & 0.266 & 0.037 & & & & \\
\hline & & 0.243 & & & 0.076 & 0.30 & 0.58 & 1.930 & 0.266 & 0.037 & & & & $F_{R_{z \phi \phi}}$ \\
\hline & & 0.365 & & & 0.076 & 0.45 & 0.87 & 1.930 & 0.266 & 0.037 & & & & \\
\hline & & 0.318 & & & 0.096 & 0.60 & 1.49 & 2.438 & 0.336 & 0.046 & & & & \\
\hline & & 0.390 & & & 0.096 & 0.75 & 1.82 & 2.438 & 0.336 & 0.046 & & & & \\
\hline \multirow[t]{9}{*}{0.280} & \multirow[t]{9}{*}{1.531} & 0.055 & & & 0.134 & 0.05 & 0.08 & 1.676 & 0.469 & 0.131 & $\mathrm{FX}_{0}$ & $\mathrm{FX}_{\sigma \tau}$ & $\mathrm{FR}_{\mathrm{z} \theta}$ & \\
\hline & & 0.164 & & & 0.134 & 0.15 & 0.25 & 1.676 & 0.469 & 0.131 & $\mathrm{FX}_{0}$ & $\mathrm{FX}_{\sigma \tau}$ & $\mathrm{FR}_{\mathrm{z} \theta}$ & \\
\hline & & 0.327 & & & 0.134 & 0.30 & 0.50 & 1.676 & 0.469 & 0.131 & $\mathbf{F X}_{\mathbf{0}}$ & $\mathbf{F X}_{\boldsymbol{\sigma} \tau}$ & $F R_{z \theta}$ & $\mathbf{F R}_{z \theta \phi}$ \\
\hline & & 0.301 & & & 0.172 & 0.45 & 0.98 & 2.152 & 0.602 & 0.169 & & $\mathrm{FX}_{\sigma \tau}$ & $\mathrm{FR}_{\mathrm{z} \theta}$ & \\
\hline & & 0.396 & & & 0.172 & 0.60 & 1.29 & 2.152 & 0.602 & 0.169 & & $\mathrm{FX}_{\sigma \tau}$ & $\mathrm{FR}_{\mathrm{z} \theta}$ & \\
\hline & & 0.377 & & & 0.200 & 0.75 & 1.93 & 2.502 & 0.700 & 0.196 & & $\mathrm{FX}_{\sigma \tau}$ & $\mathrm{FR}_{\mathrm{z} \theta}$ & \\
\hline & & 0.140 & & & 0.250 & 0.45 & 1.41 & 3.127 & 0.876 & 0.245 & $\mathrm{FX}_{0}$ & & & \\
\hline & & 0.187 & & & 0.250 & 0.60 & 1.88 & 3.127 & 0.876 & 0.245 & $\mathrm{FX}_{0}$ & & & \\
\hline & & 0.234 & & & 0.250 & 0.75 & 2.35 & 3.127 & 0.876 & 0.245 & $\mathrm{FX}_{0}$ & & & \\
\hline 0.410 & 2.241 & 0.117 & & & 0.134 & 0.05 & 0.06 & 1.145 & 0.469 & 0.192 & $\mathrm{FX}_{0}$ & & $\mathrm{FR}_{\mathrm{z} \theta}$ & \\
\hline & & 0.351 & & & 0.134 & 0.15 & 0.17 & 1.145 & 0.469 & 0.192 & $\mathrm{FX}_{0}$ & & $\mathrm{FR}_{\mathrm{z} \theta}$ & \\
\hline & & 0.220 & & & 0.239 & 0.30 & 0.61 & 2.042 & 0.837 & 0.343 & $\mathbf{F X}_{\mathbf{0}}$ & & $\mathbf{F R}_{\mathbf{z} \theta}$ & $\mathbf{F R}_{z \theta \phi}$ \\
\hline & & 0.330 & & & 0.239 & 0.45 & 0.92 & 2.042 & 0.837 & 0.343 & $\mathrm{FX}_{0}$ & & $\mathrm{FR}_{\mathrm{z \theta}}$ & \\
\hline & & & & & & $\underline{Y a w-a l}$ & drift & & & & & & & \\
\hline $\begin{array}{l}F r \\
(-)\end{array}$ & $\begin{array}{c}U_{\mathrm{C}} \\
(\mathrm{m} / \mathrm{s})\end{array}$ & $\begin{array}{l}\beta \\
\left.{ }^{\circ}\right)\end{array}$ & $\begin{array}{c}y_{\max } \\
(\mathrm{m})\end{array}$ & $\begin{array}{c}\psi_{\max } \\
\left(^{\circ}\right)\end{array}$ & $\begin{array}{c}f \\
(\mathrm{~Hz})\end{array}$ & $\begin{array}{r}r_{\max } \\
(-)\end{array}$ & $\begin{array}{c}\dot{r}_{\max } \\
(-)\end{array}$ & $\begin{array}{l}\omega_{1}^{\prime} \\
(-)\end{array}$ & $\begin{array}{l}\omega_{2}^{\prime} \\
(-)\end{array}$ & $\begin{array}{l}\omega_{3}^{\prime} \\
(-)\end{array}$ & & Mount & onditior & \\
\hline 0.280 & 1.531 & 9 & 0.327 & 10.2 & 0.134 & 0.30 & 0.50 & 1.676 & 0.469 & 0.131 & $\mathrm{FX}_{0}$ & & $\mathrm{FR}_{\mathrm{z} \theta}$ & \\
\hline & & 10 & 0.327 & 10.2 & 0.134 & 0.30 & 0.50 & 1.676 & 0.469 & 0.131 & $\mathbf{F X}_{\mathbf{0}}$ & & $\mathbf{F R}_{\mathrm{z} \theta}$ & $\mathbf{F R}_{z \theta \phi}$ \\
\hline & & 11 & 0.327 & 10.2 & 0.134 & 0.30 & 0.50 & 1.676 & 0.469 & 0.131 & $\mathrm{FX}_{0}$ & & $\mathrm{FR}_{\mathrm{z} \theta}$ & \\
\hline
\end{tabular}

Note: Bold $=\mathrm{UA}$ case $;{ }^{1)} \mathrm{UA}$ case for $\mathrm{FR}_{z \theta}$ and $\mathrm{FR}_{\mathrm{z} \theta \phi} ;{ }^{2)} \mathrm{UA}$ case for $\mathrm{FX}_{0}$ and $\mathrm{FX}_{\sigma \tau}$; Shaded $=$ PIV cases in Part II. 
Table 7 Summary of statistical convergence study $\left(F r=0.280, \mathrm{FR}_{\mathrm{z} \theta \phi}\right)$.

\begin{tabular}{|c|c|c|c|c|c|c|}
\hline \multirow{2}{*}{ Variable } & \multirow{2}{*}{$\begin{array}{l}\text { Dominant frequencies } \\
(\mathrm{Hz})\end{array}$} & \multicolumn{2}{|c|}{$\begin{array}{l}\text { Reverse Arrangement, } A \\
\quad(65 \leq A \leq 125)^{\dagger}\end{array}$} & \multirow{2}{*}{$\begin{array}{l}\text { Chi-square, } X^{2} \\
\left(X^{2} \leq 51\right)^{\dagger}\end{array}$} & \multicolumn{2}{|c|}{ Convergence Error, $E_{\mathrm{SC}}(\%)$} \\
\hline & & $\bar{x}$ & $\overline{x^{2}}$ & & $c=2$ & $c=4.5$ \\
\hline \multicolumn{7}{|c|}{ Static drift $\left(\beta=10^{\circ} ; M=12\right)$} \\
\hline$U_{C}$ & $0.1,0.2,0.5,1.27,2.53$ & $134[1]$ & 108 [ 9] & $420[0]$ & $0.03\langle 0.03\rangle$ & $0.06\langle 0.06\rangle$ \\
\hline$F_{x}$ & $0.8,1.27,2.53,4,5,8,10$ & 99 [12] & $115[10]$ & $41[8]$ & $1.21\langle 1.45\rangle$ & $3.73\langle 3.25\rangle$ \\
\hline$F_{y}$ & $0.8,4,5,8,10$ & $125[11]$ & $101[10]$ & $46[5]$ & $0.42\langle 0.44\rangle$ & $0.93\langle 0.99\rangle$ \\
\hline$M_{z}$ & 5,8 & $132[7]$ & $119[10]$ & $63[4]$ & $0.26\langle 0.26\rangle$ & $0.58\langle 0.59\rangle$ \\
\hline$Z_{\mathrm{mm}}$ & $0.25,1.27,18$ & $101[11]$ & $115[6]$ & $65[0]$ & $0.38\langle 0.39\rangle$ & $0.85\langle 0.88\rangle$ \\
\hline$\theta$ & 0.25 & $107[12]$ & $106[9]$ & $250[0]$ & $0.35\langle 0.33\rangle$ & $0.78\langle 0.73\rangle$ \\
\hline$\phi$ & 0.13 & $150[3]$ & $81[10]$ & $365[0]$ & $0.05\langle 0.05\rangle$ & $0.12\langle 0.12\rangle$ \\
\hline \multicolumn{7}{|c|}{ pure yaw $\left(r_{\max }=0.3 ; M=11\right)$} \\
\hline$F_{x}\left(A_{2}\right)$ & $0.268,4,5$ & $173[4]$ & $102[10]$ & $1,032[0]$ & $0.52\langle 0.50\rangle$ & $1.16\langle 1.11\rangle$ \\
\hline$F_{y}\left(A_{1}\right)$ & $0.134,4,5,7,8,10$ & $22[2]$ & $96[11]$ & $562[0]$ & $0.01\langle 0.02\rangle$ & $0.03\langle 0.03\rangle$ \\
\hline$M_{z}\left(A_{1}\right)$ & $0.134,4,8$ & $95[5]$ & $109[11]$ & $620[0]$ & $0.01\langle 0.04\rangle$ & $0.03\langle 0.09\rangle$ \\
\hline$Z_{\mathrm{mm}}\left(A_{2}\right)$ & $0.268,1.27$ & $70[2]$ & $92[11]$ & $558[0]$ & $0.48\langle 1.01\rangle$ & $1.07\langle 2.26\rangle$ \\
\hline$\theta\left(A_{2}\right)$ & 0.268 & $190[2]$ & 123 [ 9] & $1,188[0]$ & $0.64\langle 0.59\rangle$ & $1.44\langle 1.33\rangle$ \\
\hline$\phi\left(A_{1}\right)$ & 0.134 & 25 [ 3] & 91 [11] & $2,586[0]$ & $0.03\langle 0.03\rangle$ & $0.06\langle 0.07\rangle$ \\
\hline
\end{tabular}

${ }^{\dagger}$ Acceptance region

[ ]: Number of acceptance cases from $M$ repeat tests

\langle\rangle : Average value of repeat tests

Table 8 Bias limit estimations for DRE variables.

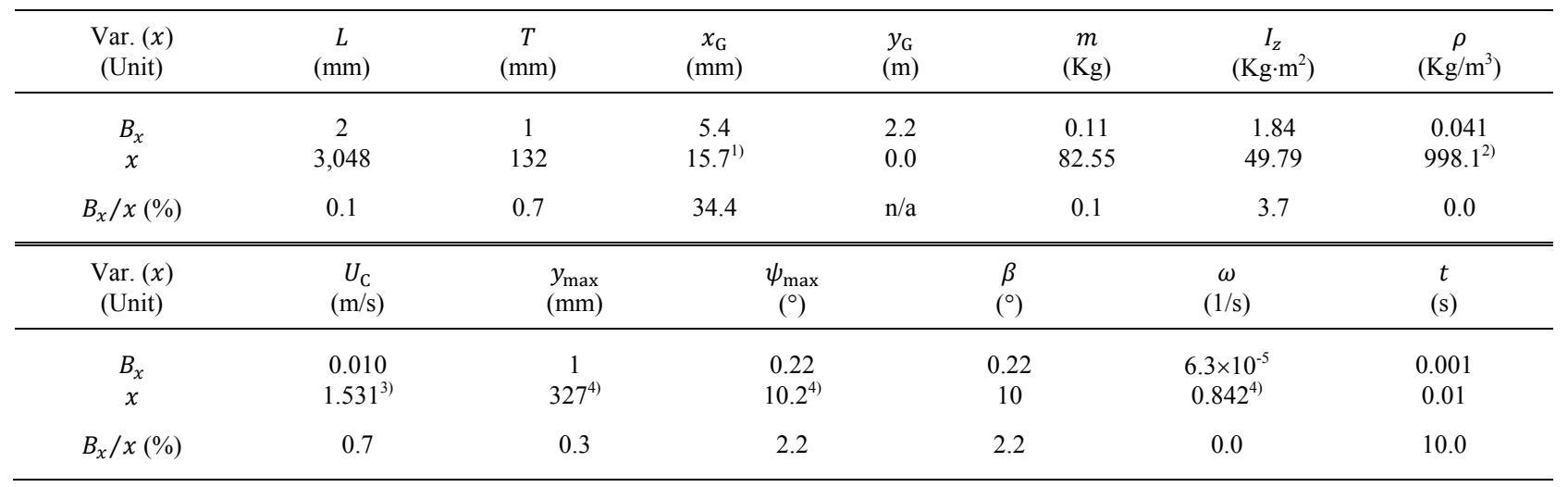

${ }^{1)}$ Absolute value; ${ }^{2)}$ Water temperature at $20{ }^{\circ} \mathrm{C} ;{ }^{3)} \mathrm{Fr}=0.280 ;{ }^{4)}$ Pure yaw $r_{\max }=0.3$ and $\mathrm{Fr}=0.280$ 
Table 9 UA summary and asymmetry bias $\left(F r=0.280, \mathrm{FR}_{z \theta}\right)$.

\begin{tabular}{|c|c|c|c|c|c|c|c|c|c|}
\hline \multicolumn{10}{|c|}{ Static drift $(\beta=109)$} \\
\hline Var. $(r)$ & $|r|$ & $\begin{array}{c}B_{r}^{2} \\
\left(\% U_{r}^{2}\right)\end{array}$ & $\begin{array}{c}P_{r}^{2} \\
\left(\% U_{r}^{2}\right) \\
\end{array}$ & $\begin{array}{c}U_{r} \\
(\%|r|)\end{array}$ & $\left|r_{\mathrm{m}}\right|$ & $\begin{array}{c}D_{\text {asym }} \\
\left(\%\left|r_{m}\right|\right)\end{array}$ & $\begin{array}{c}U_{r} \\
\left(\%\left|r_{m}\right|\right)\end{array}$ & $\begin{array}{c}B_{\text {asym }} \\
\left(\%\left|r_{m}\right|\right)\end{array}$ & $\begin{array}{c}U_{r, \text { ssym }} \\
\left(\%\left|r_{m}\right|\right)\end{array}$ \\
\hline$X$ & 0.023 & 96.6 & 3.4 & 1.9 & 0.021 & 10.5 & 2.1 & 10.3 & 10.5 \\
\hline$Y$ & 0.061 & 95.1 & 4.9 & 3.4 & 0.062 & 0.4 & 3.3 & 0.0 & 3.3 \\
\hline$N$ & 0.031 & 94.5 & 5.5 & 2.8 & 0.031 & 0.6 & 2.8 & 0.0 & 2.8 \\
\hline$z\left(10^{-2}\right)$ & 0.296 & 99.4 & 0.6 & 10.0 & 0.288 & 2.8 & 10.3 & 0.0 & 10.3 \\
\hline$\theta\left({ }^{\circ}\right)$ & 0.152 & 99.9 & 0.1 & 26.4 & 0.212 & 28.5 & 18.9 & 21.4 & 28.5 \\
\hline \multicolumn{10}{|c|}{ Pure yaw $\left(r_{\max }=0.3\right)$} \\
\hline Var. $(r)$ & $D_{r}^{*}$ & $\begin{array}{c}\overline{B_{r}^{2}} \\
\left(\% U_{r}^{2}\right)\end{array}$ & $\begin{array}{c}\overline{P_{r}^{2}} \\
\left(\% U_{r}^{2}\right)\end{array}$ & $\begin{array}{c}\overline{U_{r}} \\
\left(\% D_{r}\right)\end{array}$ & $D_{r, \mathrm{FS}}$ & $\begin{array}{c}\overline{D_{\text {asym }}} \\
\left(\% D_{r, \mathrm{FS}}\right)\end{array}$ & $\begin{array}{c}\overline{U_{r}} \\
\left(\% D_{r, \mathrm{FS}}\right)\end{array}$ & $\begin{array}{c}\overline{B_{\text {asym }}} \\
\left(\% D_{r, \mathrm{FS}}\right)\end{array}$ & $\begin{array}{c}\overline{U_{r, \text { asym }}} \\
\left(\% D_{r, \mathrm{FS}}\right)\end{array}$ \\
\hline$X$ & 0.019 & 19.0 & 81.0 & 7.6 & 0.019 & 9.5 & 7.6 & 5.9 & 10.4 \\
\hline$Y$ & 0.034 & 74.4 & 25.6 & 3.7 & 0.034 & 3.2 & 3.7 & 1.7 & 4.7 \\
\hline$N$ & 0.031 & 81.1 & 18.9 & 1.2 & 0.031 & 1.6 & 1.2 & 1.0 & 1.6 \\
\hline$z\left(10^{-2}\right)$ & 1.540 & 91.4 & 8.6 & 20.1 & 1.540 & 18.2 & 20.1 & 0.0 & 20.1 \\
\hline$\theta\left({ }^{\circ}\right)$ & 0.127 & 77.8 & 22.2 & 35.7 & 0.127 & 33.4 & 35.7 & 17.1 & 42.9 \\
\hline
\end{tabular}

Table $10 \mathrm{UA}$ comparisons between facilities and Facility Bias $\left(F r=0.280, \mathrm{FR}_{z \theta}\right)$.

\begin{tabular}{|c|c|c|c|c|c|c|c|c|c|c|c|c|c|}
\hline \multirow[t]{2}{*}{ Var. } & \multirow[t]{2}{*}{ Facility } & \multicolumn{4}{|c|}{$\begin{array}{c}\text { N-order level } \\
\left(\% X_{i}\right)\end{array}$} & \multicolumn{4}{|c|}{$\begin{array}{c}\mathrm{M} \times \mathrm{N} \text {-order level } \\
(\% \bar{X})\end{array}$} & \multicolumn{4}{|c|}{$\begin{array}{l}\text { Facility Certification or biases } \\
(\% \bar{X})\end{array}$} \\
\hline & & $X_{i}$ & $B_{X_{i}}^{2}$ & $P_{X_{i}}^{2}$ & $U_{X_{i}}$ & $U_{X_{i}}$ & $B_{\bar{X}}^{2}$ & $P_{\bar{X}}^{2}$ & $U_{\bar{X}}$ & $D_{i}$ & $U_{D_{i}}$ & $U_{F B_{i}}$ & $U_{T_{i}}$ \\
\hline \multicolumn{14}{|c|}{ Static drift $(\beta=109$} \\
\hline \multirow{4}{*}{$X$} & $\mathrm{~A}$ & 0.0214 & 99.9 & 0.1 & 10.5 & 11.5 & \multirow{4}{*}{98.6} & \multirow{4}{*}{1.4} & \multirow{4}{*}{5.2} & 9.4 & 12.6 & 0.0 & 11.5 \\
\hline & B & 0.0199 & 77.8 & 22.2 & 3.3 & 3.4 & & & & 1.7 & 6.2 & 0.0 & 3.4 \\
\hline & $\mathrm{C}$ & 0.0174 & 99.1 & 0.9 & 11.5 & 10.2 & & & & 11.1 & 11.5 & 0.0 & 10.2 \\
\hline & Avg. & 0.0196 & 92.3 & 7.7 & 8.4 & 8.4 & & & & 7.4 & 10.1 & 0.0 & 8.4 \\
\hline \multirow[t]{4}{*}{$Y$} & A & 0.0619 & 95.0 & 5.0 & 3.3 & 3.3 & \multirow{4}{*}{73.0} & \multirow{4}{*}{27.0} & \multirow{4}{*}{1.7} & 0.6 & 3.7 & 0.0 & 3.3 \\
\hline & B & 0.0607 & 74.2 & 25.8 & 2.1 & 2.1 & & & & 1.4 & 2.7 & 0.0 & 2.1 \\
\hline & $\mathrm{C}$ & 0.0620 & 52.0 & 48.0 & 3.4 & 3.4 & & & & 0.8 & 3.8 & 0.0 & 3.4 \\
\hline & Avg. & 0.0615 & 73.7 & 26.3 & 2.9 & 2.9 & & & & 0.9 & 3.4 & 0.0 & 2.9 \\
\hline \multirow[t]{4}{*}{$N$} & $\mathrm{~A}$ & 0.0313 & 94.8 & 5.2 & 2.8 & 2.9 & \multirow{4}{*}{62.2} & \multirow{4}{*}{37.8} & \multirow{4}{*}{1.7} & 4.3 & 3.4 & 2.7 & 4.0 \\
\hline & B & 0.0297 & 46.4 & 53.6 & 3.0 & 3.0 & & & & 1.0 & 3.4 & 0.0 & 3.0 \\
\hline & $\mathrm{C}$ & 0.0290 & 48.5 & 51.5 & 3.3 & 3.2 & & & & 3.3 & 3.6 & 0.0 & 3.2 \\
\hline & Avg. & 0.0300 & 63.2 & 36.8 & 3.0 & 3.0 & & & & 2.9 & 3.5 & 0.9 & 3.4 \\
\hline \multicolumn{14}{|c|}{ Pure yaw $\left(r_{\max }=0.3 ;\right.$ at $\left.r=r_{\max }\right)$} \\
\hline \multirow[t]{4}{*}{$X$} & A & 0.0187 & 74.2 & 25.8 & 14.9 & 15.4 & \multirow{4}{*}{78.6} & \multirow{4}{*}{21.4} & \multirow{4}{*}{5.7} & 3.3 & 16.4 & 0.0 & 15.4 \\
\hline & B & 0.0187 & 98.8 & 1.2 & 3.4 & 3.4 & & & & 3.4 & 6.7 & 0.0 & 3.5 \\
\hline & $\mathrm{C}$ & 0.0168 & 97.6 & 2.4 & 6.9 & 6.4 & & & & 6.7 & 8.6 & 0.0 & 6.4 \\
\hline & Avg. & 0.0181 & 90.2 & 9.8 & 8.4 & 8.4 & & & & 4.5 & 10.6 & 0.0 & 8.4 \\
\hline \multirow[t]{4}{*}{$Y$} & A & 0.0170 & 87.9 & 12.1 & 10.2 & 9.9 & \multirow{4}{*}{89.0} & \multirow{4}{*}{11.0} & \multirow{4}{*}{4.2} & 3.0 & 10.7 & 0.0 & 9.9 \\
\hline & B & 0.0178 & 93.4 & 6.6 & 5.5 & 5.6 & & & & 1.4 & 7.0 & 0.0 & 5.6 \\
\hline & $\mathrm{C}$ & 0.0178 & 87.9 & 12.1 & 5.3 & 5.4 & & & & 1.6 & 6.8 & 0.0 & 5.4 \\
\hline & Avg. & 0.0175 & 89.7 & 10.3 & 7.0 & 7.0 & & & & 2.0 & 8.2 & 0.0 & 7.0 \\
\hline \multirow[t]{4}{*}{$N$} & A & 0.0151 & 92.9 & 7.1 & 3.3 & 3.3 & & & & 0.4 & 3.7 & 0.0 & 3.3 \\
\hline & B & 0.0140 & 95.7 & 4.3 & 3.8 & 3.5 & 91.4 & 8.6 & 1.7 & 6.7 & 3.9 & 5.5 & 6.5 \\
\hline & $\mathrm{C}$ & 0.0160 & 59.6 & 40.4 & 1.4 & 1.5 & & & & 6.3 & 2.3 & 5.9 & 6.1 \\
\hline & Avg. & 0.0150 & 82.7 & 17.3 & 2.8 & 2.8 & & & & 4.5 & 3.3 & 3.8 & 5.3 \\
\hline
\end{tabular}


Table 11 Hydrodynamic Derivatives $\left(\mathrm{FR}_{z \theta}\right)$.

\begin{tabular}{|c|c|c|c|c|c|c|c|c|c|c|c|}
\hline \multirow{2}{*}{$X$} & \multicolumn{3}{|c|}{$\mathrm{Fr}$} & \multirow{2}{*}{$Y$} & \multicolumn{3}{|c|}{$\mathrm{Fr}$} & \multirow{2}{*}{$N$} & \multicolumn{3}{|c|}{$\mathrm{Fr}$} \\
\hline & 0.138 & 0.280 & 0.410 & & 0.138 & 0.280 & 0.410 & & 0.138 & 0.280 & 0.410 \\
\hline \multicolumn{12}{|c|}{ Static drift test } \\
\hline $\begin{array}{c}X_{*} \\
X_{v v}\end{array}$ & $\begin{array}{l}-0.0182 \\
-0.0301\end{array}$ & $\begin{array}{l}-0.0170 \\
-0.1528\end{array}$ & $\begin{array}{l}-0.0258 \\
-0.1544\end{array}$ & $\begin{array}{c}Y_{v} \\
Y_{v v v}\end{array}$ & $\begin{array}{l}-0.2637 \\
-1.6256\end{array}$ & $\begin{array}{l}-0.2961 \\
-1.9456\end{array}$ & $\begin{array}{l}-0.2963 \\
-3.7914\end{array}$ & $\begin{array}{c}N_{v} \\
N_{v v v}\end{array}$ & $\begin{array}{l}-0.1396 \\
-0.3426\end{array}$ & $\begin{array}{l}-0.1667 \\
-0.4355\end{array}$ & $\begin{array}{l}-0.1717 \\
-1.2591\end{array}$ \\
\hline $\begin{array}{c}X_{u} \\
X_{u u} \\
X_{v v u}\end{array}$ & & $\begin{array}{l}-0.0088 \\
-0.0220 \\
-0.1172\end{array}$ & & $\begin{array}{c}Y_{v u} \\
Y_{v u u}\end{array}$ & & $\begin{array}{c}-0.0307 \\
0.0653\end{array}$ & & $\begin{array}{c}N_{v u} \\
N_{v u u}\end{array}$ & & $\begin{array}{c}-0.0311 \\
0.0439\end{array}$ & \\
\hline \multicolumn{12}{|c|}{ Dynamic test (MR $R_{L}$ method) } \\
\hline $\begin{array}{c}X_{*} \\
X_{v v}\end{array}$ & & $\begin{array}{l}-0.0173 \\
-0.4765\end{array}$ & & $\begin{array}{c}Y_{v} \\
Y_{v v v} \\
Y_{\dot{v}}\end{array}$ & & $\begin{array}{l}-0.2601 \\
-2.9686 \\
-0.1135\end{array}$ & & $\begin{array}{c}N_{v} \\
N_{v v v} \\
N_{\dot{v}}\end{array}$ & & $\begin{array}{l}-0.1681 \\
-0.5677 \\
-0.0136\end{array}$ & \\
\hline $\begin{array}{c}X_{*} \\
X_{r r}\end{array}$ & $\begin{array}{l}-0.0181 \\
-0.0078\end{array}$ & $\begin{array}{l}-0.0177 \\
-0.0282\end{array}$ & $\begin{array}{l}-0.0260 \\
-0.0385\end{array}$ & $\begin{array}{c}Y_{r} \\
Y_{r r r} \\
Y_{\dot{r}}\end{array}$ & $\begin{array}{l}-0.0276 \\
-0.0370 \\
-0.0146\end{array}$ & $\begin{array}{l}-0.0485 \\
-0.0452 \\
-0.0090\end{array}$ & $\begin{array}{l}-0.0548 \\
-0.0710 \\
-0.0127\end{array}$ & $\begin{array}{c}N_{r} \\
N_{r r r} \\
N_{\dot{r}}\end{array}$ & $\begin{array}{l}-0.0382 \\
-0.0211 \\
-0.0065\end{array}$ & $\begin{array}{l}-0.0485 \\
-0.0505 \\
-0.0070\end{array}$ & $\begin{array}{l}-0.0557 \\
-0.0821 \\
-0.0077\end{array}$ \\
\hline$X_{v r}$ & & 0.0819 & & $\begin{array}{l}Y_{v r r} \\
Y_{r v v}\end{array}$ & & $\begin{array}{l}-2.0198 \\
-1.8819\end{array}$ & & $\begin{array}{l}N_{v r r} \\
N_{r v v}\end{array}$ & & $\begin{array}{l}-0.6891 \\
-0.4367\end{array}$ & \\
\hline$X_{r r u}$ & & -0.0308 & & $\begin{array}{c}Y_{r u} \\
Y_{r u u}\end{array}$ & & $\begin{array}{r}-0.0268 \\
0.0284\end{array}$ & & $\begin{array}{c}N_{r u} \\
N_{r u u}\end{array}$ & & $\begin{array}{l}-0.0183 \\
-0.0066\end{array}$ & \\
\hline \multicolumn{12}{|c|}{ Dynamic test (MR $R_{H}$ method) } \\
\hline $\begin{array}{l}X_{v v} \\
X_{r r}\end{array}$ & -0.0016 & $\begin{array}{l}-0.1296 \\
-0.0132\end{array}$ & -0.0163 & $\begin{array}{l}Y_{v v v} \\
Y_{r r r} \\
Y_{v r r}\end{array}$ & -0.0927 & $\begin{array}{l}-2.2962 \\
-0.1305 \\
-0.9066\end{array}$ & -0.1210 & $\begin{array}{l}N_{v v v} \\
N_{r r r} \\
N_{v r r}\end{array}$ & -0.0312 & $\begin{array}{l}-0.8533 \\
-0.0473 \\
-0.3161\end{array}$ & -0.0387 \\
\hline
\end{tabular}

Table 12 Reconstruction Errors, $\overline{E_{R}}(\%)\left(F r=0.280, \mathrm{FR}_{\mathrm{z} \theta}\right)$.

\begin{tabular}{|c|c|c|c|c|c|c|c|c|c|}
\hline \multirow{4}{*}{ Pure sway } & Var. & $\mathrm{MR}_{\mathrm{L}}$ & $\mathrm{MR}_{\mathrm{H}}$ & $\mathrm{SR}_{2^{\circ}}$ & $\mathrm{SR}_{4^{\circ}}$ & $\mathrm{SR}_{10^{\circ}}$ & & & \\
\hline & $X$ & 9.4 & 12.0 & 22.8 & 9.4 & 19.5 & & & \\
\hline & $Y$ & 5.5 & 6.2 & 5.1 & 10.1 & 7.3 & & & \\
\hline & $N$ & 2.9 & 4.0 & 30.2 & 6.1 & 3.9 & & & \\
\hline \multirow{4}{*}{ Pure yaw } & Var. & $\mathrm{MR}_{\mathrm{L}}$ & $\mathrm{MR}_{\mathrm{H}}$ & $\mathrm{SR}_{0.05}$ & $\mathrm{SR}_{0.15}$ & $\mathrm{SR}_{0.30}$ & $\mathrm{SR}_{0.45}$ & $\mathrm{SR}_{0.60}$ & $\mathrm{SR}_{0.75}$ \\
\hline & $X$ & 7.6 & 9.5 & 549.4 & 52.2 & 12.1 & 8.5 & 9.2 & 13.8 \\
\hline & $Y$ & 17.2 & 29.5 & 106.2 & 56.3 & 25.2 & 27.2 & 31.2 & 45.7 \\
\hline & $N$ & 5.2 & 5.2 & 616.2 & 22.4 & 5.3 & 6.6 & 5.5 & 6.7 \\
\hline \multirow{4}{*}{ Yaw and drift } & Var. & $\mathrm{MR}_{\mathrm{L}}$ & $\mathrm{MR}_{\mathrm{H}}$ & $\mathrm{SR}_{\mathrm{L} 9^{\circ}}$ & $\mathrm{SR}_{\mathrm{L} 10^{\circ}}$ & $\mathrm{SR}_{\mathrm{L} 11^{\circ}}$ & $\mathrm{SR}_{\mathrm{H} 9^{\circ}}$ & $\mathrm{SR}_{\mathrm{H} 10^{\circ}}$ & $\mathrm{SR}_{\mathrm{H} 11^{\circ}}$ \\
\hline & $X$ & 11.0 & - & 11.1 & 11.0 & 11.0 & - & - & - \\
\hline & $Y$ & 3.5 & 3.5 & 3.6 & 5.2 & 5.5 & 3.6 & 3.5 & 3.7 \\
\hline & $N$ & 2.7 & 3.0 & 2.4 & 2.4 & 4.6 & 2.9 & 2.5 & 3.6 \\
\hline
\end{tabular}


Table 13 Polynomial-fit coefficients of static drift heave, pitch, and roll.

\begin{tabular}{cccccccc}
\hline & \multicolumn{4}{c}{$\underline{z\left(\times 10^{2}\right)}$} & \multicolumn{2}{c}{$\underline{\theta\left({ }^{\circ}\right)}$} & \multicolumn{2}{c}{$\phi\left({ }^{\circ}\right)$} \\
Mount & $F r$ & $A$ & $B$ & $A$ & $B$ & $A$ & $B$ \\
\hline \multirow{2}{*}{$\mathrm{FR}_{z \theta}$} & 0.138 & 0.006 & 0.284 & -0.039 & -0.153 & - & - \\
& 0.280 & 0.176 & 0.983 & -0.097 & -1.326 & - & - \\
& 0.410 & 0.434 & 2.692 & 0.396 & -2.769 & - & - \\
$\mathrm{FR}_{z \theta \phi}$ & 0.138 & -0.017 & 0.291 & -0.004 & -1.759 & 0.056 & 0.096 \\
& 0.280 & 0.217 & 0.810 & -0.026 & -6.875 & 0.119 & 0.139 \\
& 0.410 & 0.516 & 2.428 & 0.453 & -10.609 & 0.239 & 0.115 \\
\hline
\end{tabular}

Table 14 Polynomial-fit coefficients of pure yaw heave and pitch harmonics $\left(\mathrm{FR}_{z \theta}\right)$.

\begin{tabular}{ccccccc}
\hline & & \multicolumn{2}{c}{$z_{0}, \theta_{0}$} & & \multicolumn{2}{c}{$z_{2}, \theta_{2}$} \\
\cline { 3 - 4 } \cline { 5 - 6 } Var. & $F r$ & $A$ & $B$ & & $C_{0}$ & $C$ \\
\hline \multirow{2}{*}{$Z\left(\times 10^{-2}\right)$} & 0.138 & 0.006 & 0.042 & & 0.001 & 0.026 \\
& 0.280 & 0.176 & 0.228 & & 0.012 & 0.157 \\
& 0.410 & 0.434 & 0.915 & & 0.007 & 0.355 \\
$\theta\left({ }^{\circ}\right)$ & 0.138 & -0.039 & 0.011 & & 0.002 & 0.049 \\
& 0.280 & -0.097 & -0.173 & & 0.043 & 0.128 \\
& 0.410 & 0.396 & 0.520 & & 0.020 & 0.182 \\
\hline
\end{tabular}

Table 15 Hydrodynamic derivatives $\left(\mathrm{FX}_{0}, \mathrm{FX}_{\sigma \tau}\right.$, and $\mathrm{FR}_{\mathrm{z} \theta \phi}$ at $\left.F r=0.280\right)$.

\begin{tabular}{|c|c|c|c|c|c|c|c|c|c|c|c|}
\hline \multirow{2}{*}{$X$} & \multicolumn{3}{|c|}{ Mount condition } & \multirow{2}{*}{$Y$} & \multicolumn{3}{|c|}{ Mount condition } & \multirow{2}{*}{$N$} & \multicolumn{3}{|c|}{ Mount condition } \\
\hline & $\mathrm{FX}_{0}$ & $\mathrm{FX}_{\sigma \tau}$ & $\mathrm{FR}_{z \theta \phi}$ & & $\mathrm{FX}_{0}$ & $\mathrm{FX}_{\sigma \tau}$ & $\mathrm{FR}_{\mathrm{z} \theta \phi}$ & & $\mathrm{FX}_{0}$ & $\mathrm{FX}_{\sigma \tau}$ & $\mathrm{FR}_{\mathrm{z} \theta \phi}$ \\
\hline \multicolumn{12}{|c|}{ Static drift test } \\
\hline $\begin{array}{l}X_{*} \\
X_{v v}\end{array}$ & $\begin{array}{l}-0.0160 \\
-0.0988\end{array}$ & $\begin{array}{l}-0.0166 \\
-0.1019\end{array}$ & $\begin{array}{l}-0.0175 \\
-0.1361\end{array}$ & $\begin{array}{c}Y_{v} \\
Y_{v v v}\end{array}$ & $\begin{array}{l}-0.2833 \\
-1.1995\end{array}$ & $\begin{array}{l}-0.2972 \\
-1.2948\end{array}$ & $\begin{array}{l}-0.3029 \\
-1.7945\end{array}$ & $\begin{array}{c}N_{v} \\
N_{v v v}\end{array}$ & $\begin{array}{l}-0.1501 \\
-0.1156\end{array}$ & $\begin{array}{l}-0.1611 \\
-0.1196\end{array}$ & $\begin{array}{l}-0.1716 \\
-0.2999\end{array}$ \\
\hline \multicolumn{12}{|c|}{ Dynamic test (FX, $F X_{\sigma c} \cdot M R_{L}$ method, $F R_{z \theta \phi}: S R_{L}$ method) } \\
\hline & & & & $Y_{\dot{v}}$ & -0.0922 & -0.0986 & -0.1160 & $N_{\dot{v}}$ & -0.0075 & -0.0078 & -0.0129 \\
\hline$X_{r r}$ & -0.0200 & -0.0289 & 0.0087 & $\begin{array}{c}Y_{r} \\
Y_{r r r} \\
Y_{\dot{r}}\end{array}$ & $\begin{array}{l}-0.0474 \\
-0.0214 \\
-0.0116\end{array}$ & $\begin{array}{l}-0.0526 \\
-0.0211 \\
-0.0041\end{array}$ & $\begin{array}{l}-0.0419 \\
-0.1610 \\
-0.0064\end{array}$ & $\begin{array}{c}N_{r} \\
N_{r r r} \\
N_{\dot{r}}\end{array}$ & $\begin{array}{l}-0.0513 \\
-0.0221 \\
-0.0041\end{array}$ & $\begin{array}{l}-0.0455 \\
-0.0366 \\
-0.0056\end{array}$ & $\begin{array}{l}-0.0449 \\
-0.0534 \\
-0.0081\end{array}$ \\
\hline$X_{v r}$ & 0.0230 & - & 0.0961 & $\begin{array}{l}Y_{v r r} \\
Y_{r v v}\end{array}$ & $\begin{array}{l}-0.8949 \\
-1.2729\end{array}$ & $\begin{array}{l}- \\
-\end{array}$ & $\begin{array}{l}-1.0904 \\
-1.4148\end{array}$ & $\begin{array}{l}N_{v r r} \\
N_{r v v}\end{array}$ & $\begin{array}{l}-0.0587 \\
-0.1548\end{array}$ & $\begin{array}{l}- \\
-\end{array}$ & $\begin{array}{l}-0.2530 \\
-0.7092\end{array}$ \\
\hline
\end{tabular}




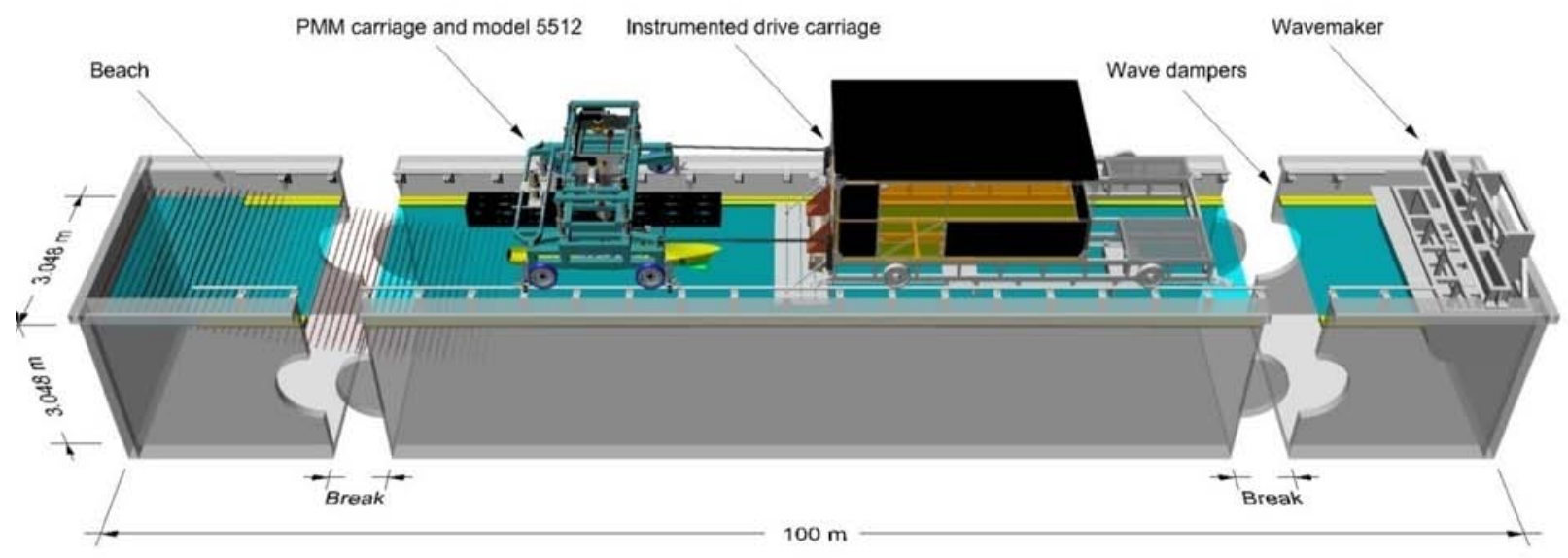

Fig. 1 IIHR towing tank facility and experimental setup for PMM test.

(a)

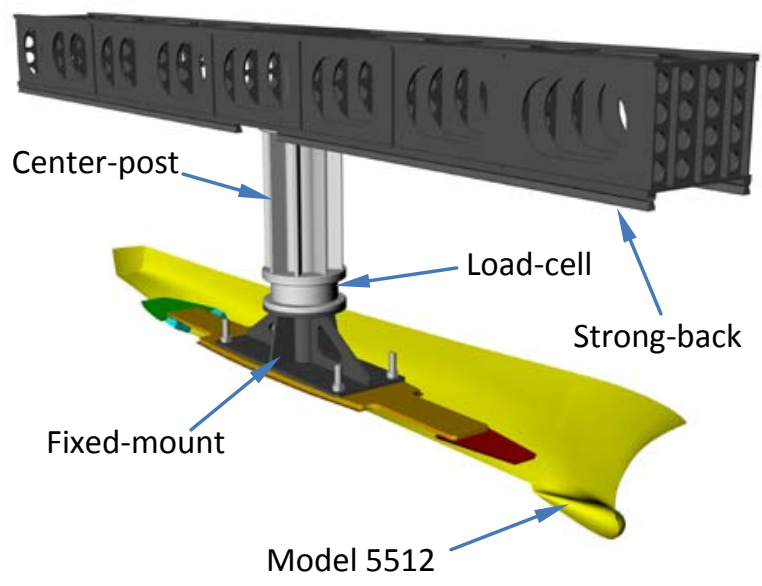

(b)

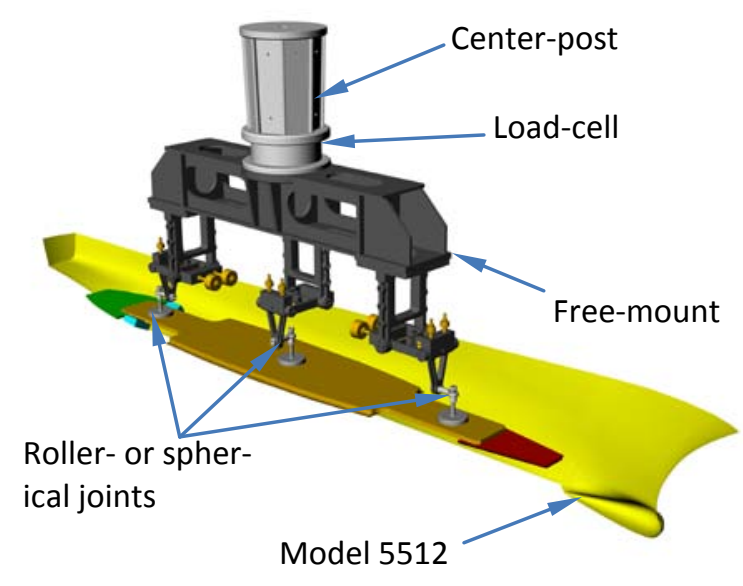

Fig. 2 (a) Fixed- and (b) free-mount setups. 
(a)

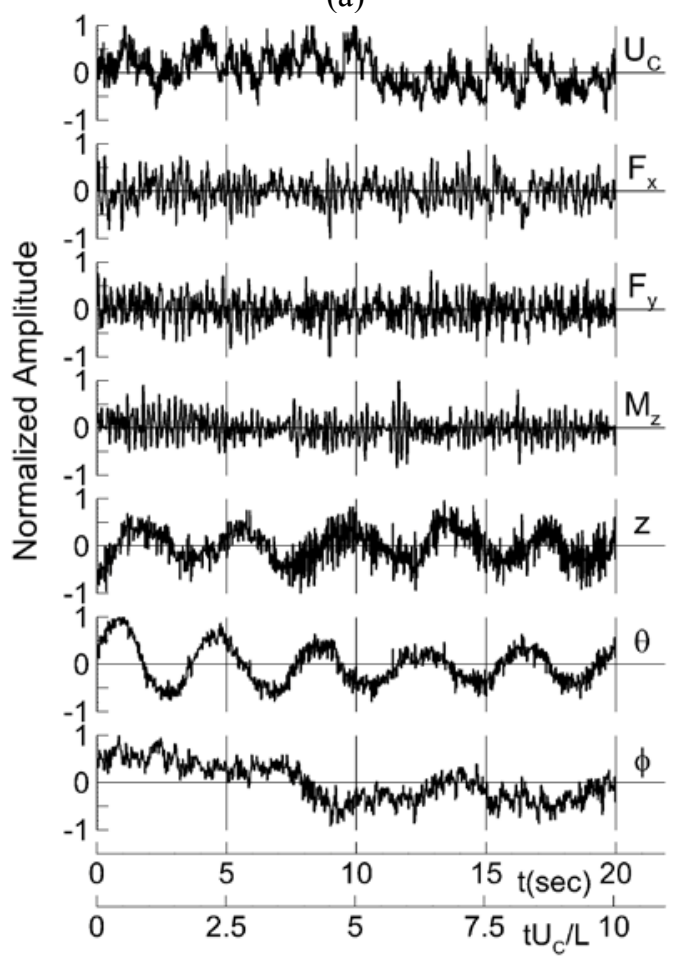

(b)

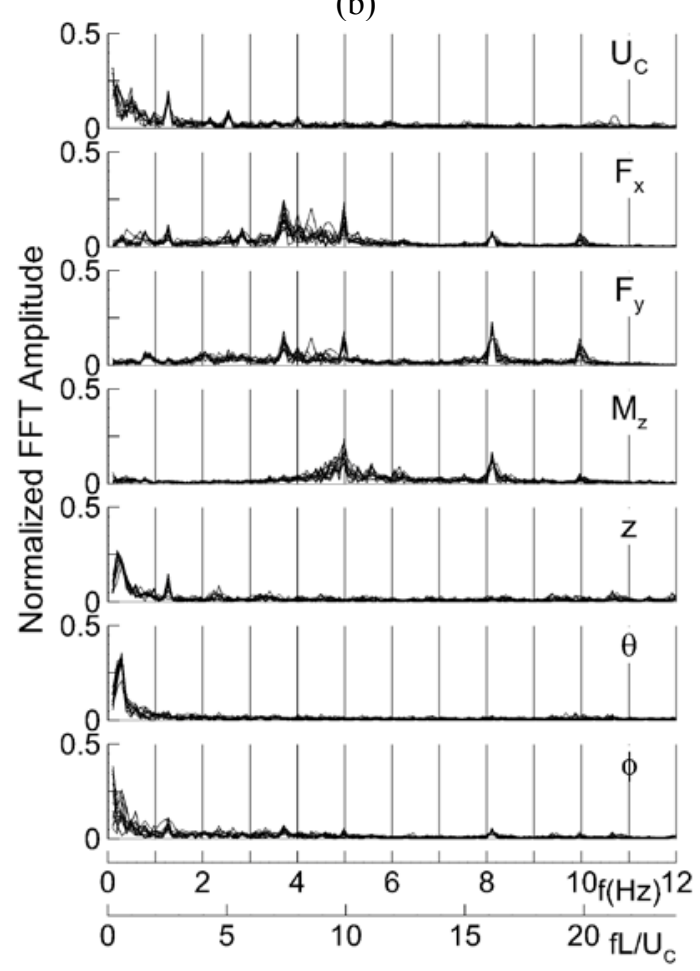

Fig. 3 Static drift variables (a) time-history and (b) FFT for 12 repeat runs $\left(\mathrm{FR}_{z \theta \phi} ; \mathrm{Fr}=0.280 ; \beta=10^{\circ}\right)$.

(a)

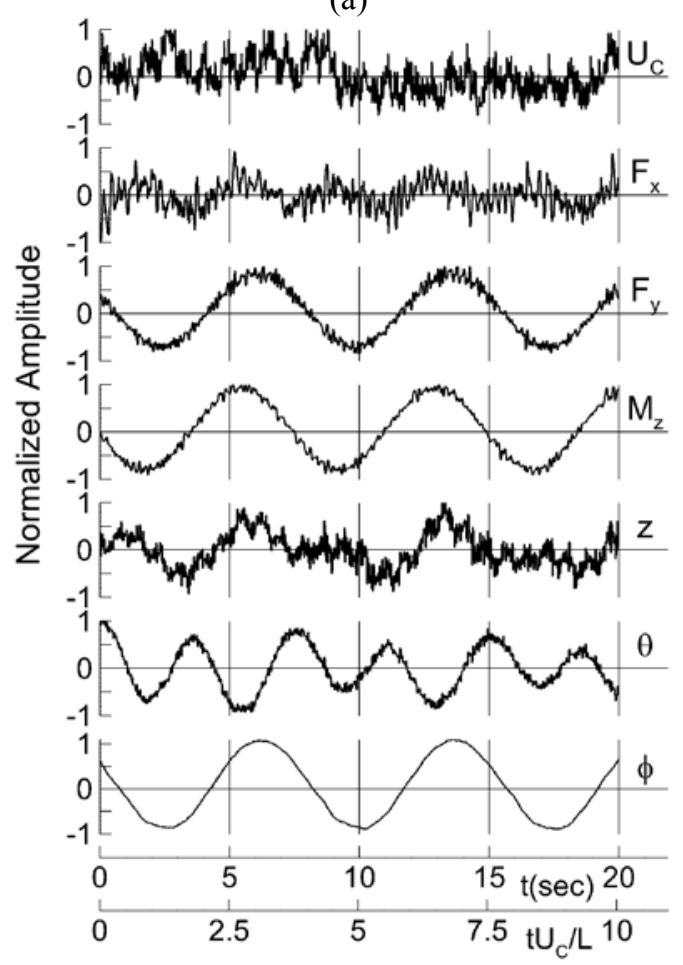

(b)

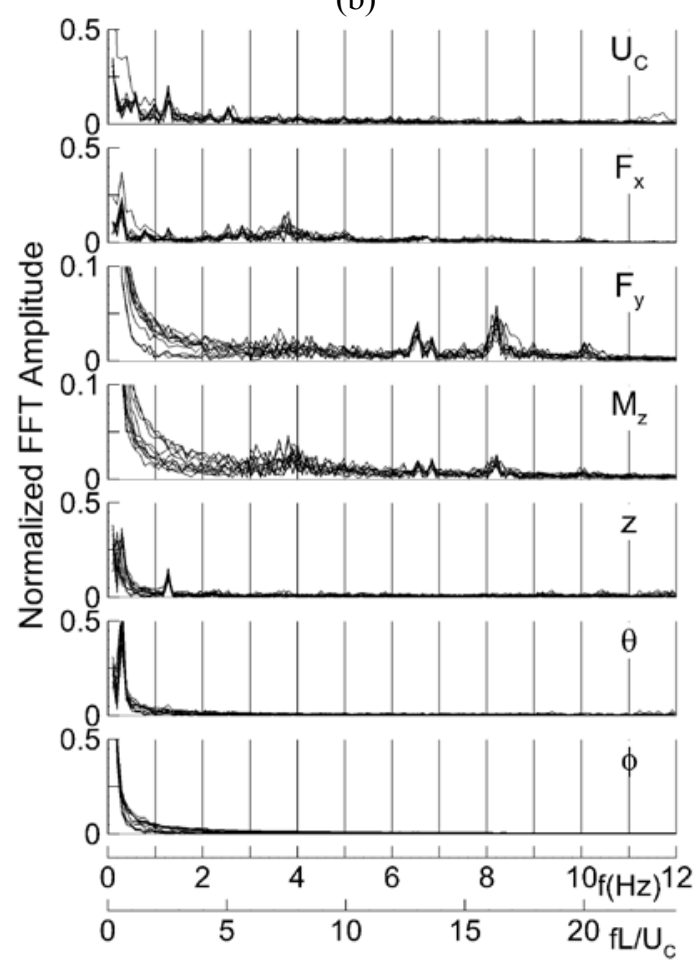

Fig. 4 Pure yaw variables (a) time history and (b) FFT for12 repeat runs $\left(\mathrm{FR}_{z \theta \phi} ; \mathrm{Fr}=0.280 ; r_{\max }=0.3\right)$. 
(a)
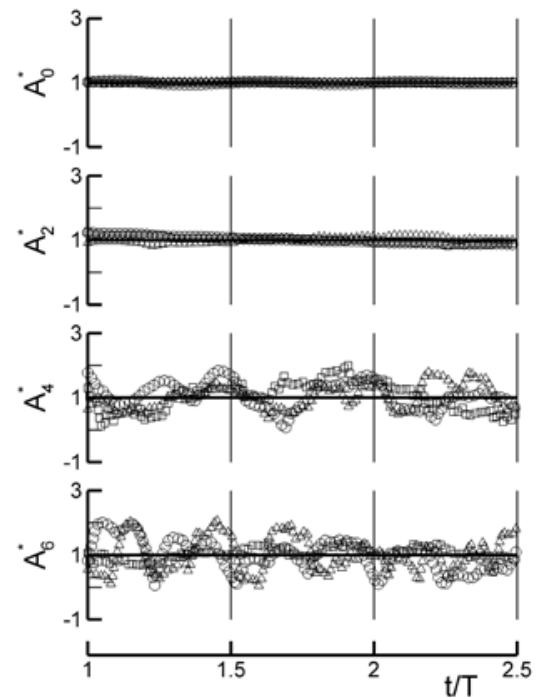

(b)
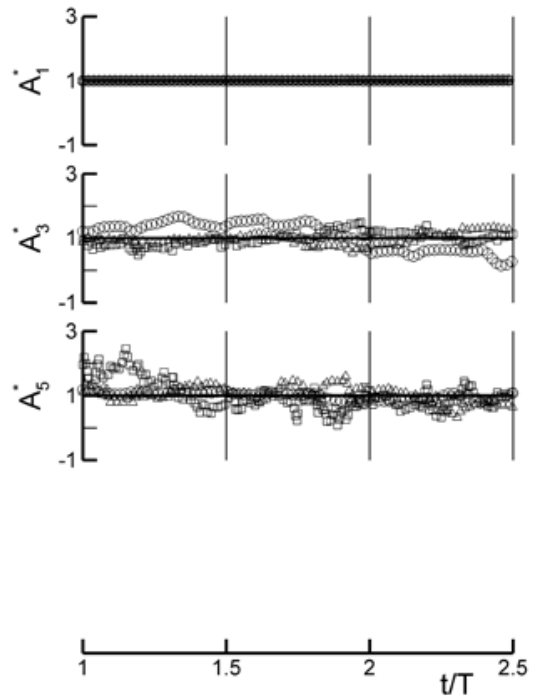

Fig. 5 Marching harmonics of pure yaw forces/moment and motions time-histories $\left(\mathrm{FR}_{z \theta \phi}, F r=0.280, r_{\max }=0.3\right)$ : (a) $F_{x}(\square), z(\Delta)$, and $\theta(\mathrm{O})$ and (b) $F_{y}(\square), M_{z}(\Delta)$, and $\phi(\mathrm{O})$.

(a)

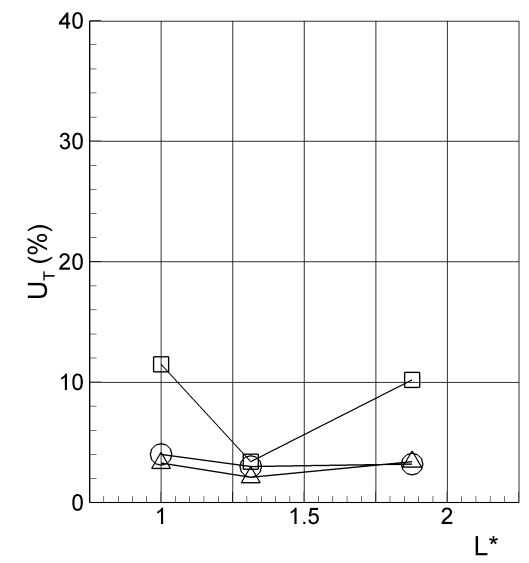

(b)

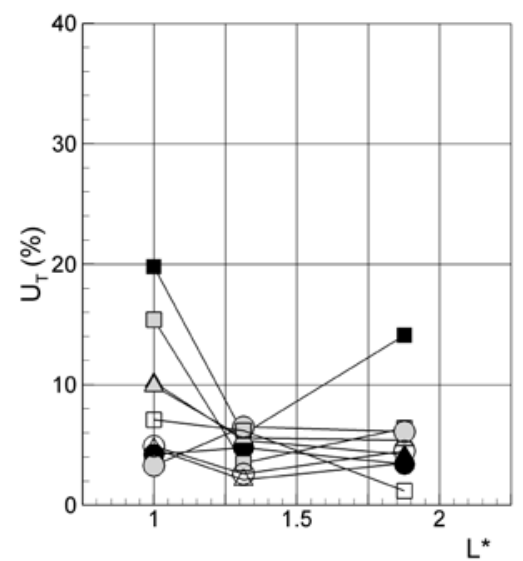

Fig. 6 Comparisons of total uncertainty $U_{T}$ among different facilities $\left(\mathrm{FR}_{z \theta \phi}\right.$ at $\left.F r=0280\right)$ : (a) Static drift and (b) dynamic PMM tests. $\mathrm{L}^{*}=1.0,1.27$, and 1.88 for facility A, B, and C, respectively. Symbols: $\square=X, \Delta=Y, \mathrm{O}=N$ for (a) and (b); Black-filled = Pure sway, Grey-filled = Pure yaw, Hollow = Yaw and drift for (b). 
(a)

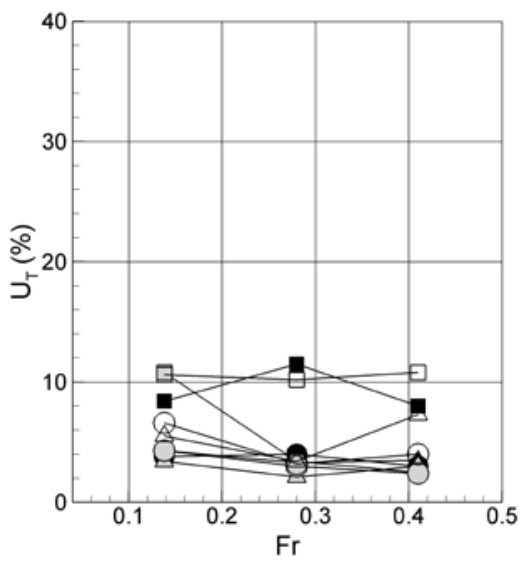

(b)

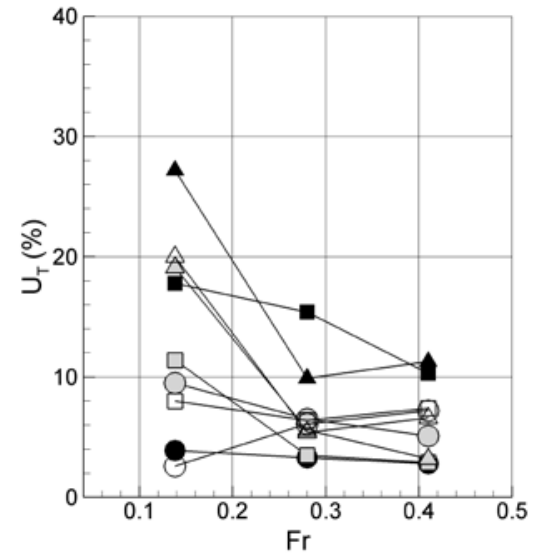

Fig. 7 Total uncertainty $U_{T}$ with $F r$ among different facilities $\left(\mathrm{FR}_{\mathrm{z} \theta}\right)$ : (a) Static drift and (b) pure yaw test. Symbols: $\square=X, \Delta=Y, \mathrm{O}=N$; Black-filled $=$ Facility A, Grey-filled $=$ Facility B, Hollow $=$ Facility $\mathrm{C}$.

(a)

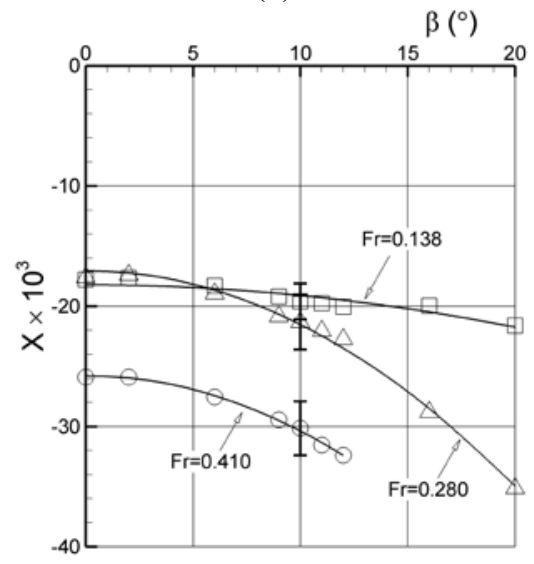

(b)

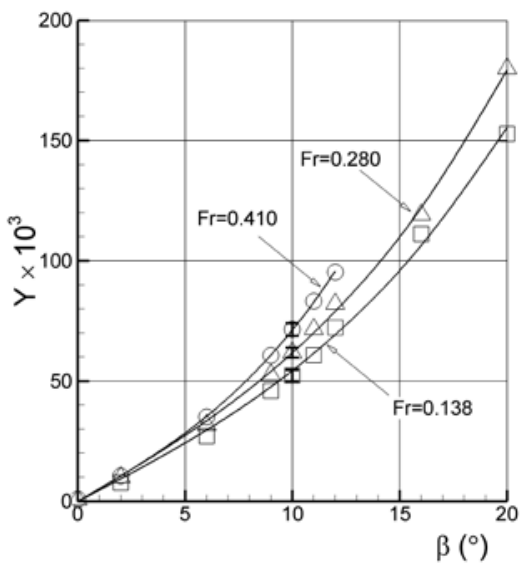

(c)

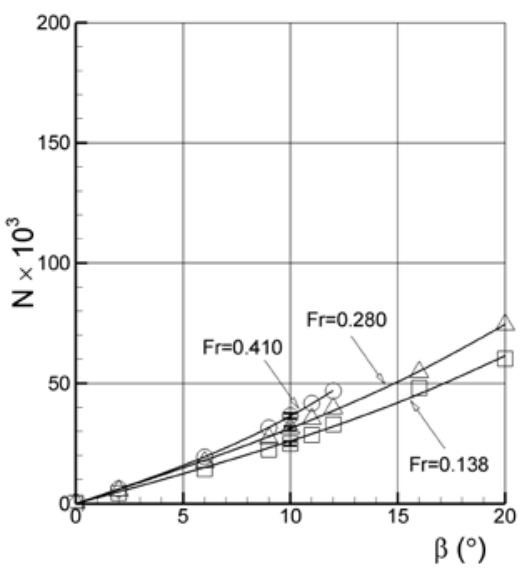

Fig. 8 Static drift (a) $X$, (b) $Y$, and (c) $N\left(\mathrm{FR}_{\mathrm{z \theta}}\right)$. 
(a)

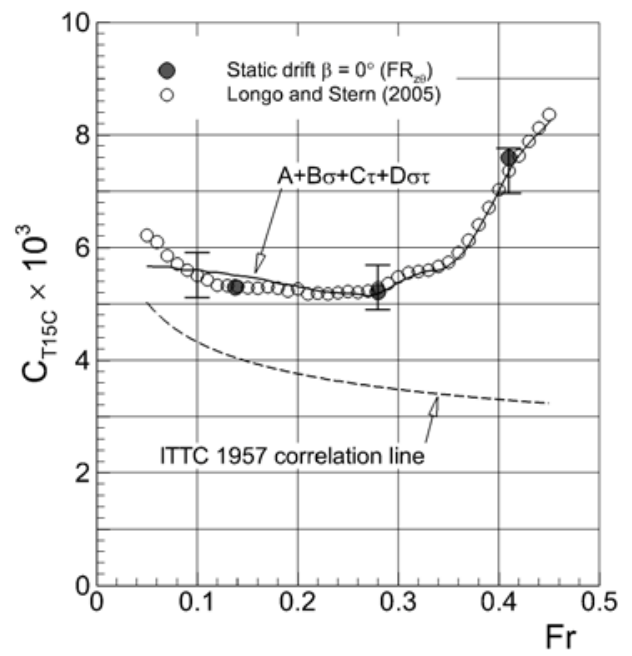

(b)

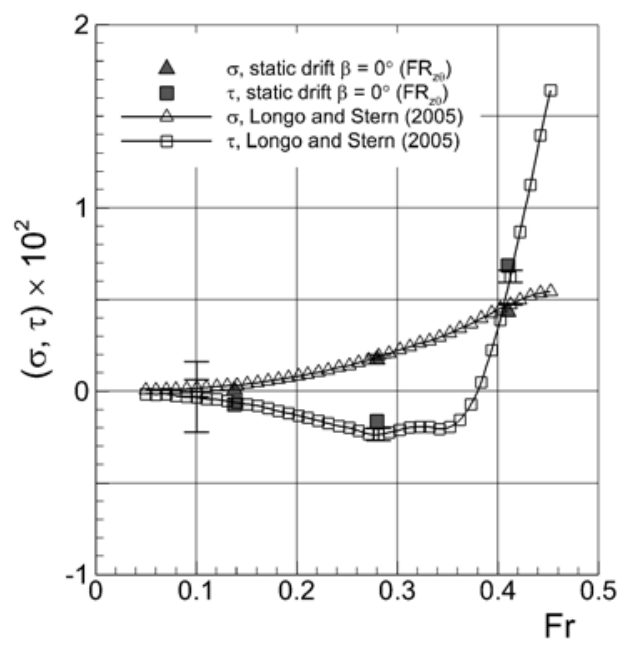

Fig. 9 Comparisons of static drift $X, z$, and $\theta$ at $\beta=0^{\circ}\left(\mathrm{FR}_{z \theta}\right)$ with resistance test by Longo and Stern (2005): (a) $C_{T 15 \mathrm{C}}$ and (b) $\sigma$ and $\tau$.

(a)

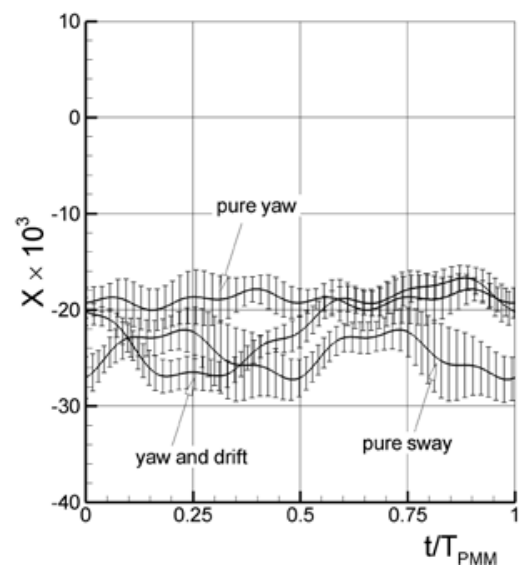

(b)

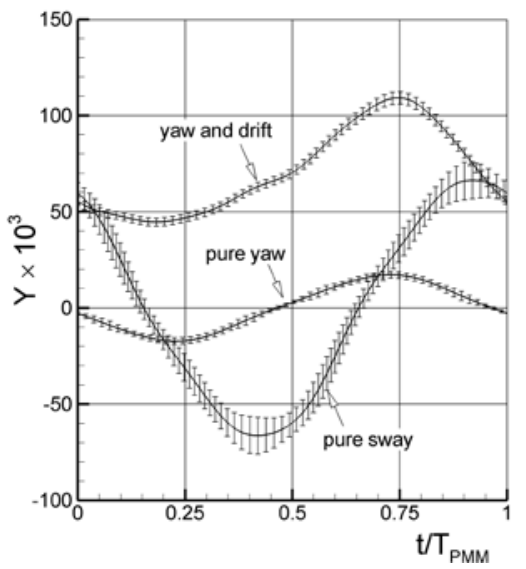

(c)

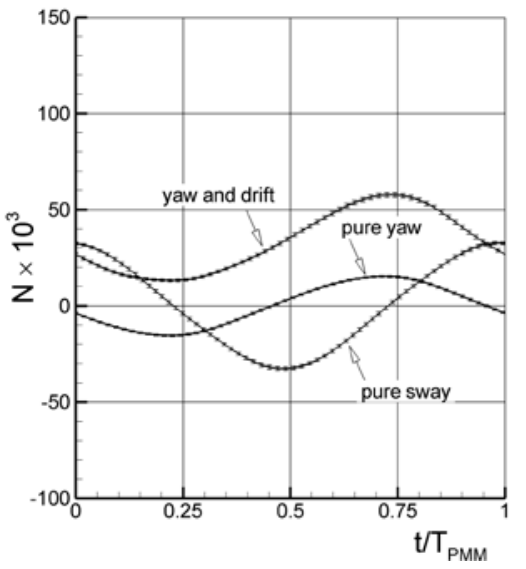

Fig. 10 Time-histories of dynamic PMM (a) $X$, (b) $Y$, and (c) $N\left(\mathrm{FR}_{z \theta \phi}\right.$ at $F r=0.280 ; \beta_{\max }=10^{\circ}$ for pure sway, $r_{\max }=0.3$ for pure yaw, and $\beta=10^{\circ}$ for yaw-and-drift). 
(a)

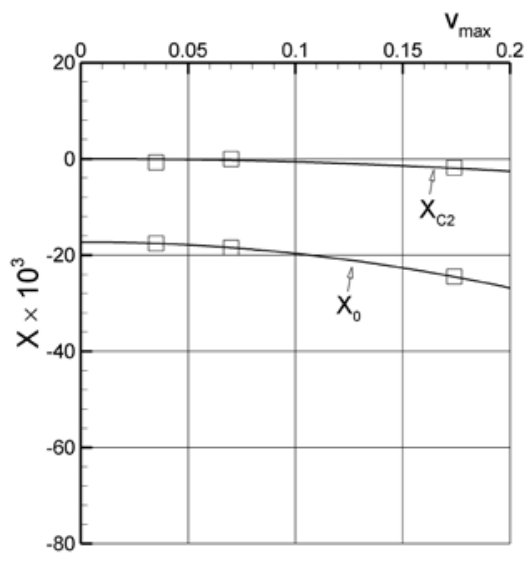

(b)

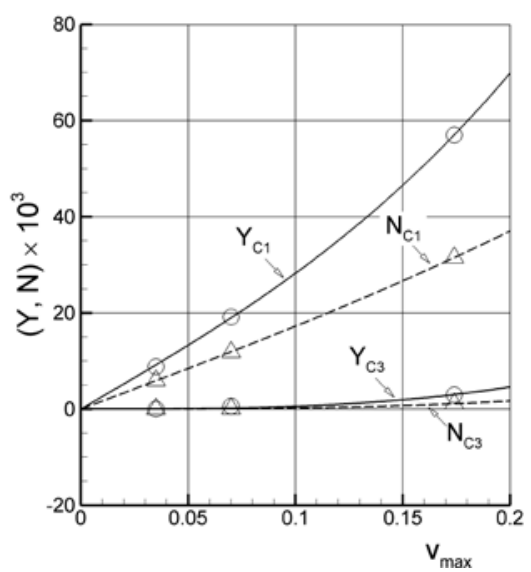

(c)

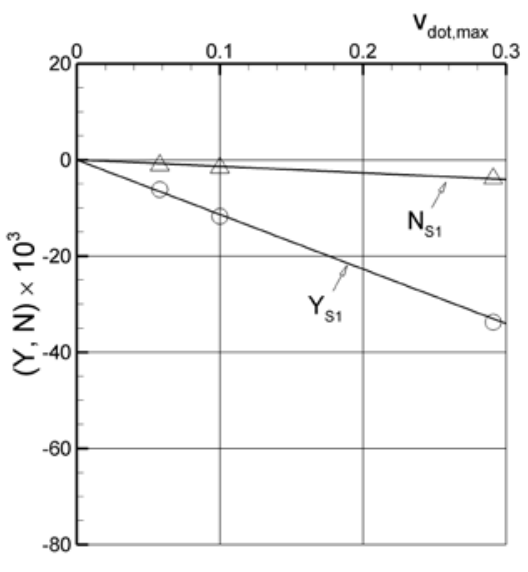

Fig. 11 Harmonic amplitudes of pure sway (a) $X$ and (b, c) $Y$ and $N\left(\mathrm{FR}_{\mathrm{z} \theta}\right)$.

(a)

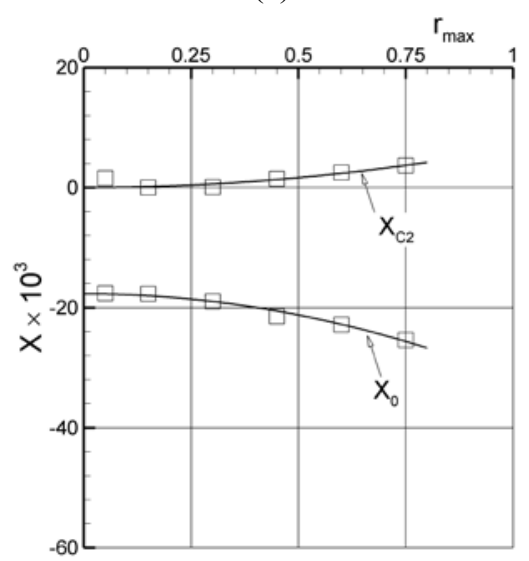

(b)

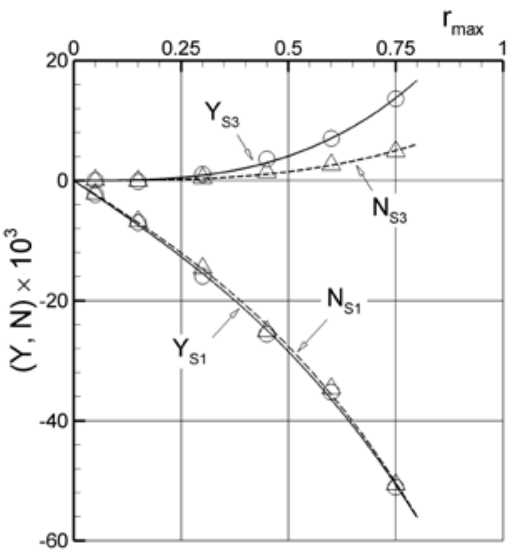

(c)

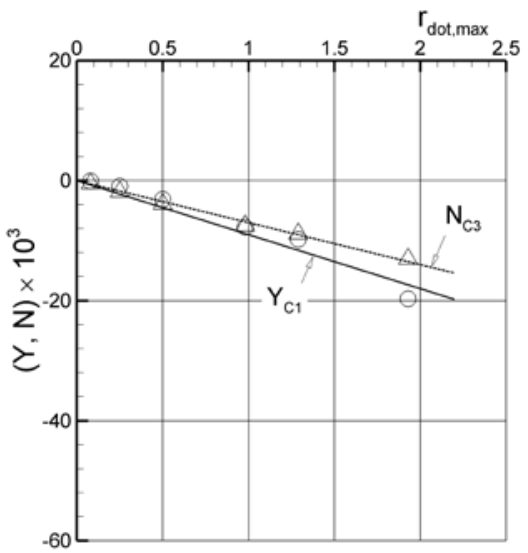

Fig. 12 Harmonic amplitudes of pure yaw (a) $X$ and (b, c) $Y$ and $N\left(\mathrm{FR}_{z \theta}\right.$ at $\left.F r=0.280\right)$.

(a)

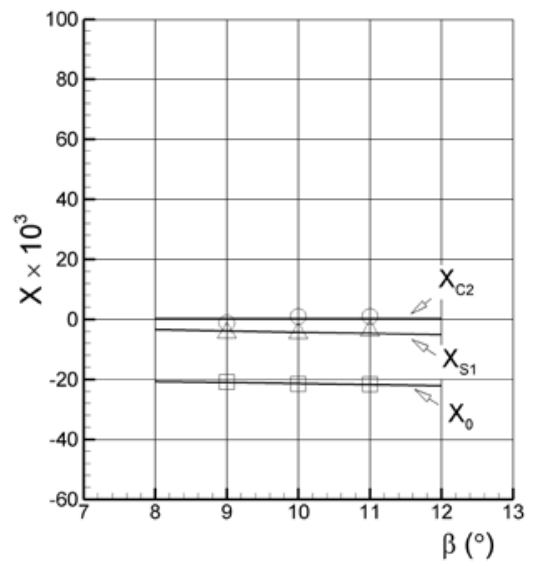

(b)

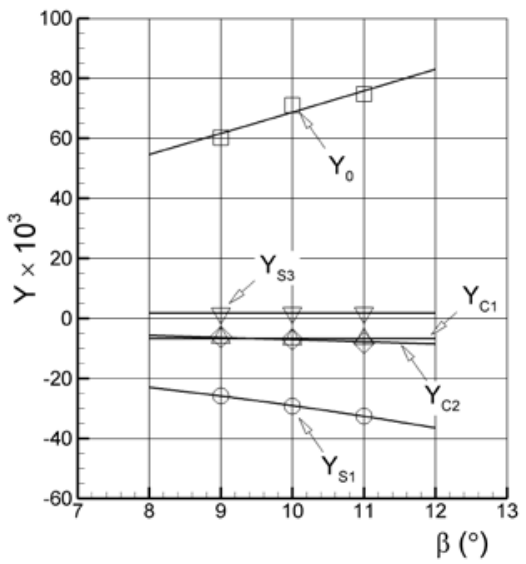

(c)

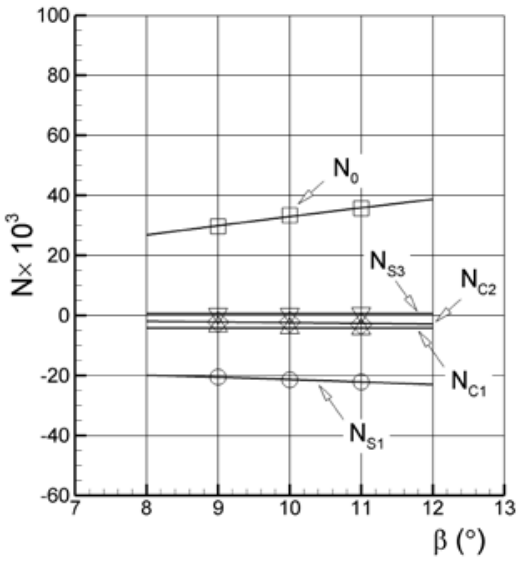

Fig. 13 Harmonic amplitudes of yaw and $\operatorname{drift}$ (a) $X$, (b) $Y$, and (c) $N\left(\mathrm{FR}_{z \theta}\right.$ at $\left.F r=0.280\right)$. 
(a)

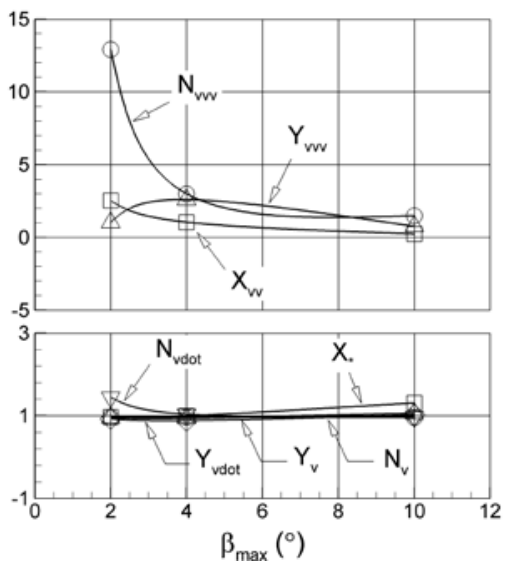

(b)

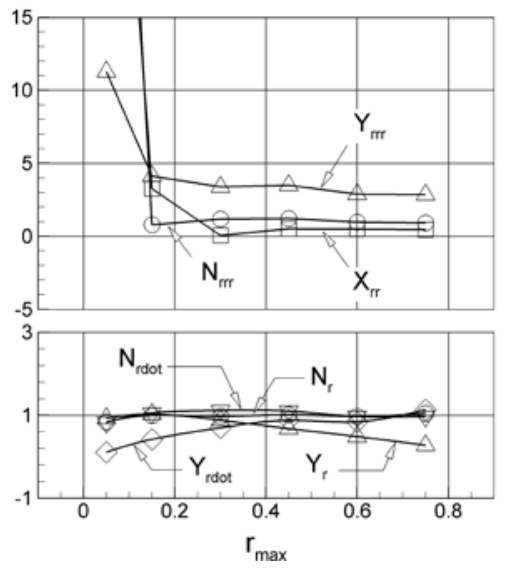

(c)

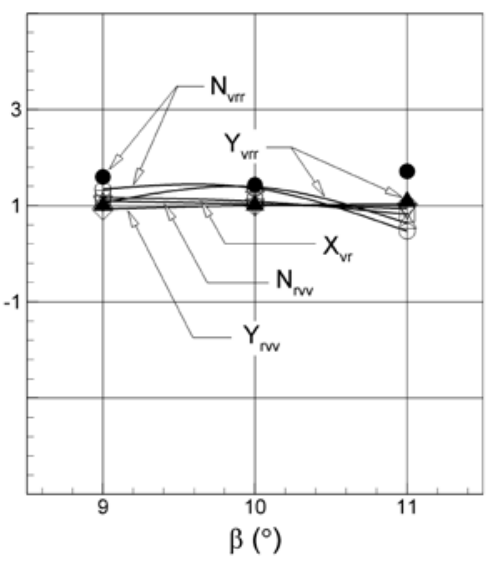

Fig. 14 Ratios of hydrodynamic derivatives by the $\mathrm{SR}_{\mathrm{L}}$ (hollow symbols) and $\mathrm{SR}_{\mathrm{H}}$ (filled symbols) methods to by the $\mathrm{MR}_{\mathrm{L}}$ method: (a) Sway-, (b) yaw-, and (c) sway-yaw-coupled derivatives $\left(\mathrm{FR}_{\mathrm{z \theta}}\right.$ at $\left.\mathrm{Fr}=0.280\right)$.

(a)

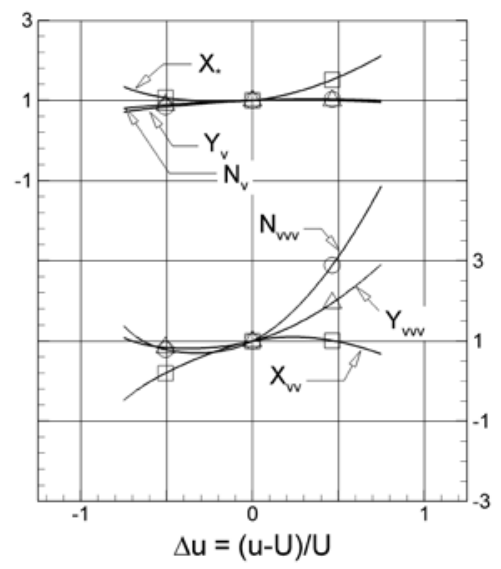

(b)

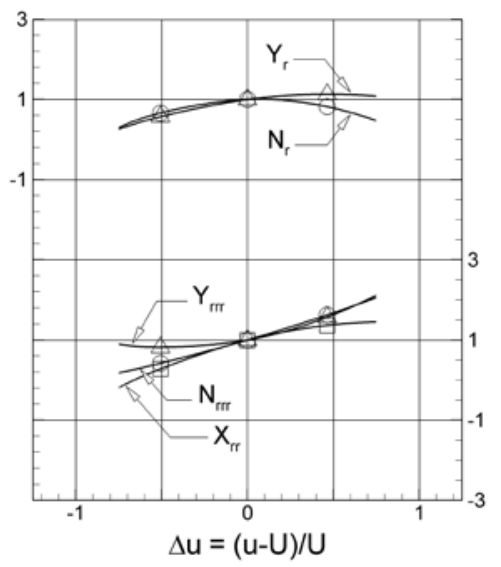

Fig. 15 Ratios of hydrodynamic derivatives of $F r=0.138,0.280$, and 0.410 to $F r=0.280\left(\mathrm{FR}_{\mathrm{z} \theta \phi}\right)$ : (a) static- and (b) rotary-derivatives. $\Delta \mathrm{u}=0.49,1.0,1.46$ for $F r=0.138,0.280$, and 0.410 , respectively. 
(a)

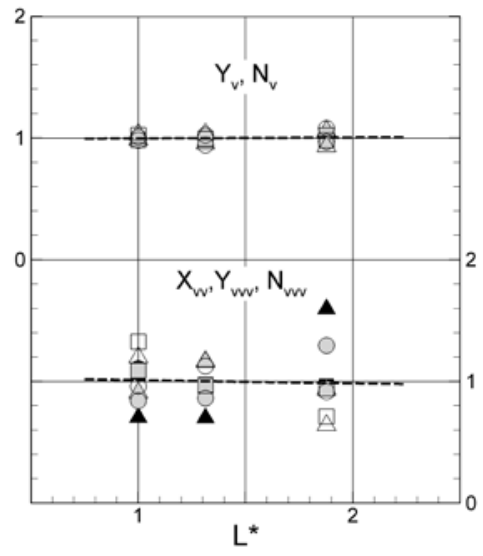

(b)

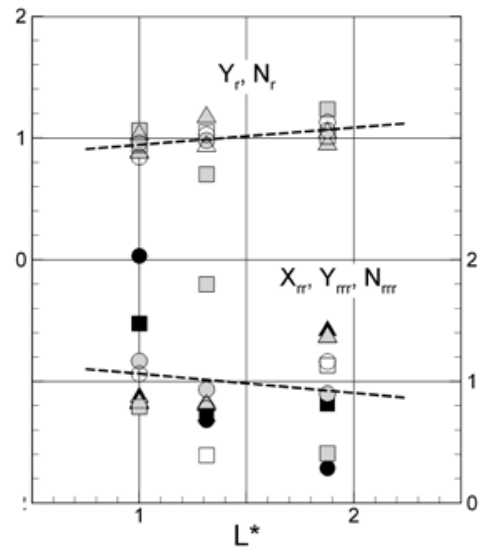

(c)

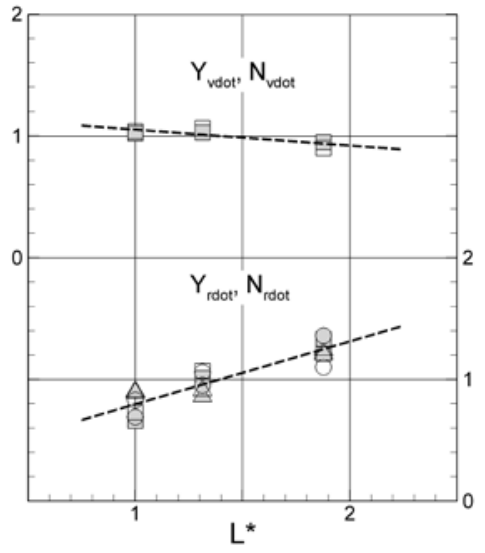

Fig. 16 Ratios of hydrodynamic derivatives of facilities A, B, and C to facility-mean (Scale effect, $\mathrm{FR}_{z \theta \phi}$ ): (a) Static, (b) rotary-, and (c) acceleration derivatives. $\mathrm{L}^{*}=1.0,1.27$, and 1.88 for facility A, B, and C, respectively. Symbols: $F r=0.138(\Delta), 0.280(\square)$, and $0.410(\mathrm{O})$; Hollow $=X$, Grey-filled $=Y$, and Black-filled $=N$ derivatives

(a)

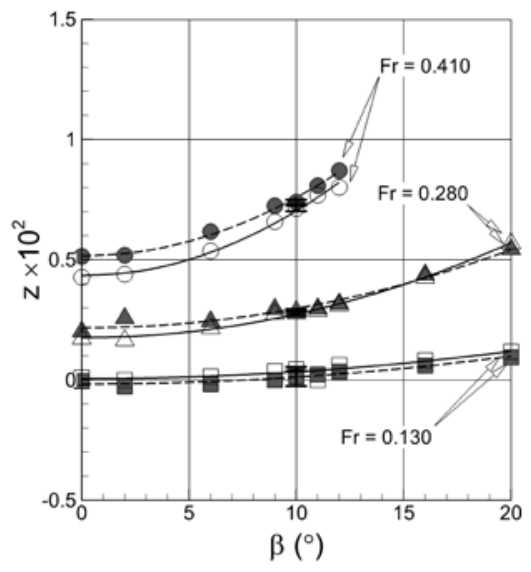

(b)

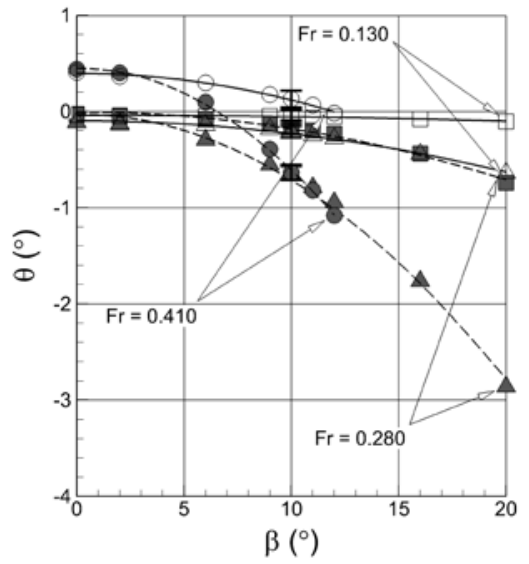

(c)

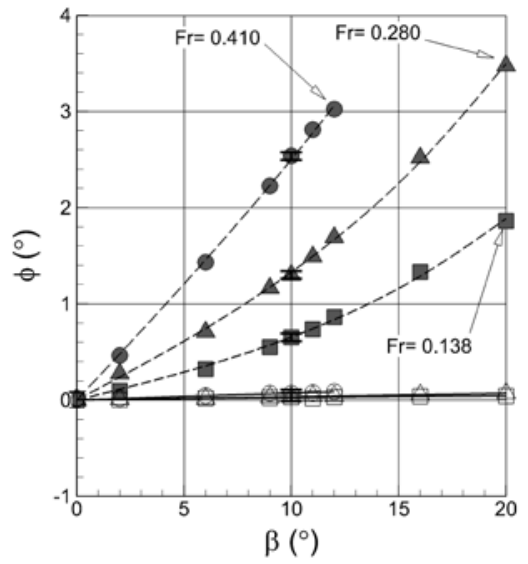

Fig. 17 Static drift heave, pitch, and roll motions: (a) $z$, (b) $\theta$, and (c) $\phi$. Symbols: $F r=0.138(\Delta), 0.280(\square)$, and $0.410(\mathrm{O})$; Solid-lines with hollow symbols $=\mathrm{FR}_{z \theta}$ and Broken-lines with grey-filled symbols $=\mathrm{FR}_{z \theta \phi}$. 
(a)

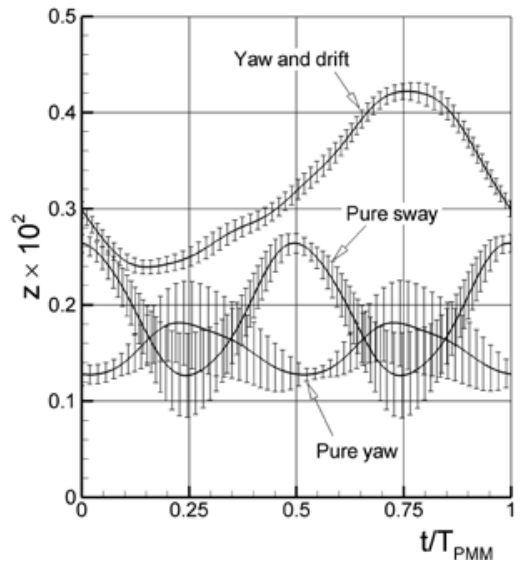

(d)

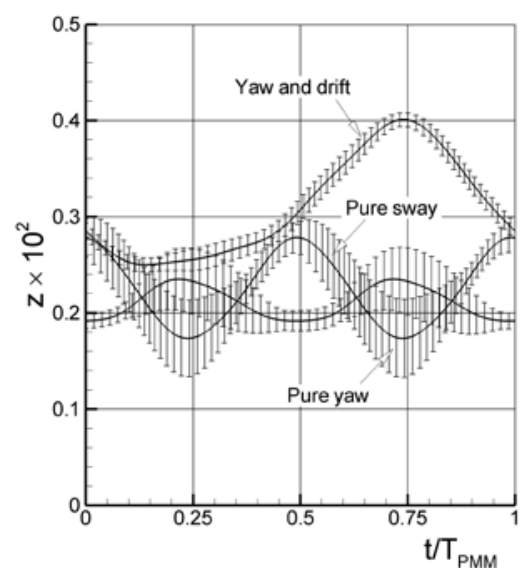

(b)

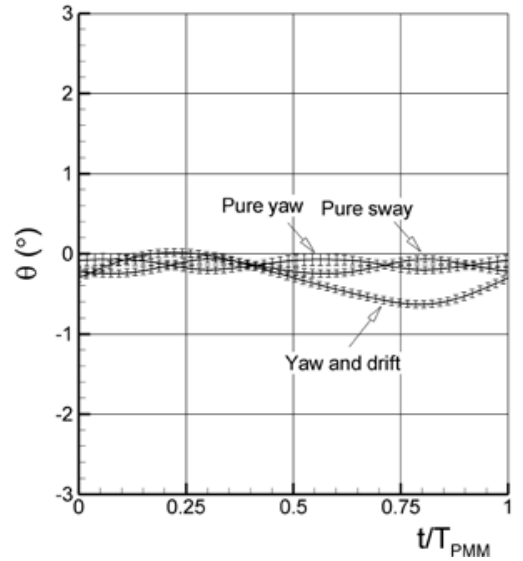

(e)

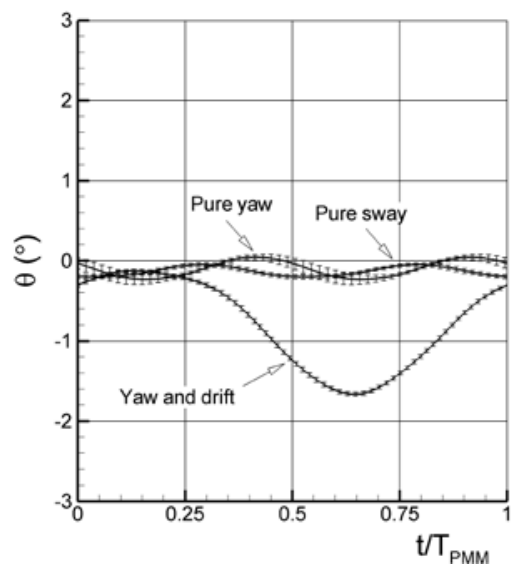

(c)

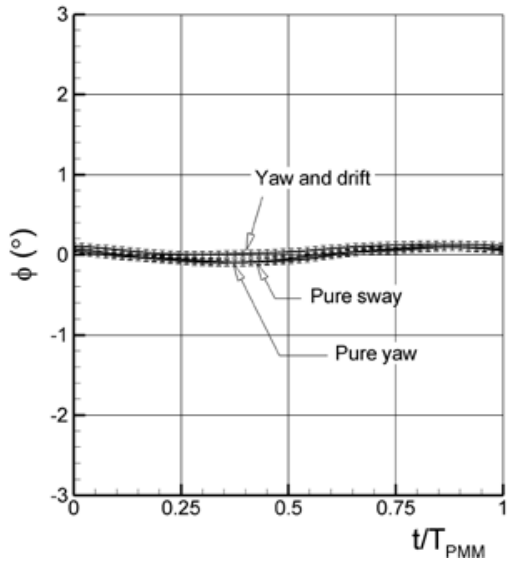

(f)

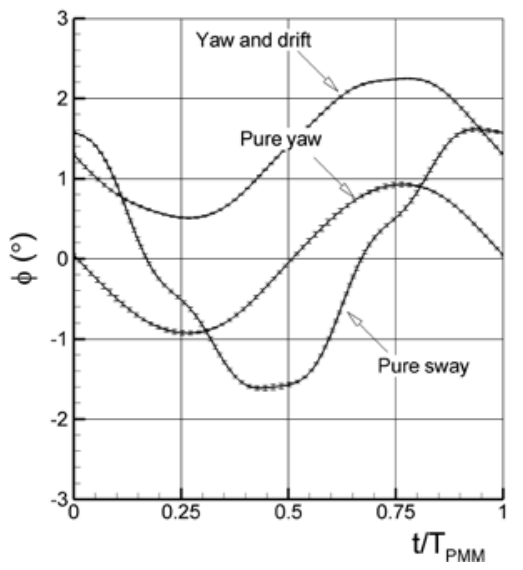

Fig. 18 Time-histories of dynamic PMM heave, pitch, and roll motions: (a) $z$, (b) $\theta$, and (c) $\phi$ of $\mathrm{FR}_{z \theta}$ and (d) $z$, (e) $\theta$, and (f) $\phi$ of $\mathrm{FR}_{z \theta \phi}(F r=0.280)$. 
(a)

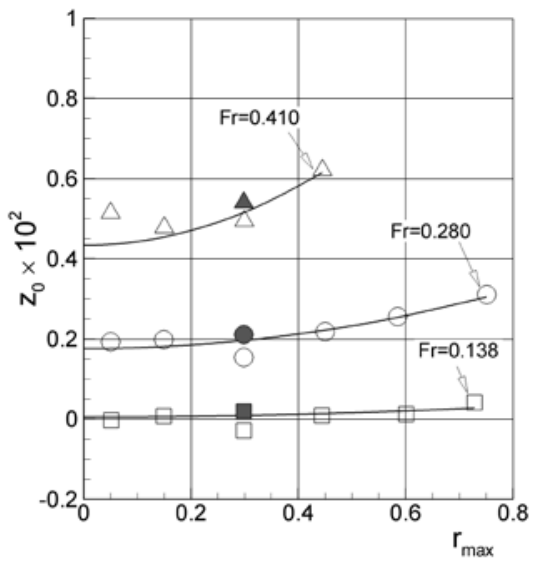

(d)

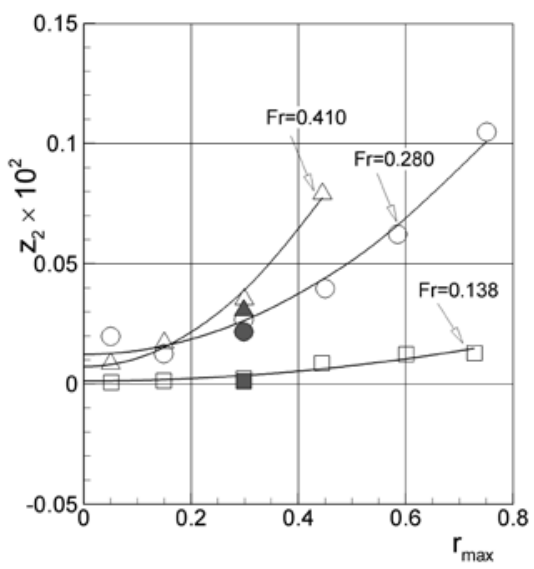

(b)

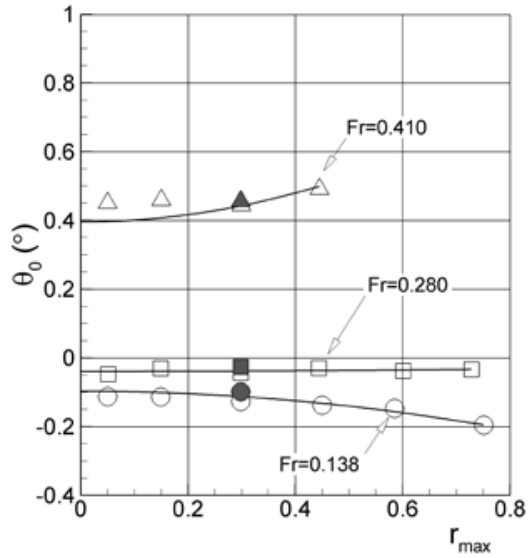

(e)

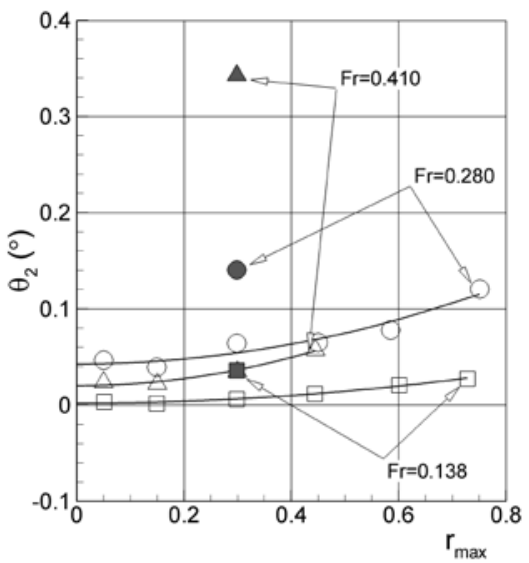

(c)

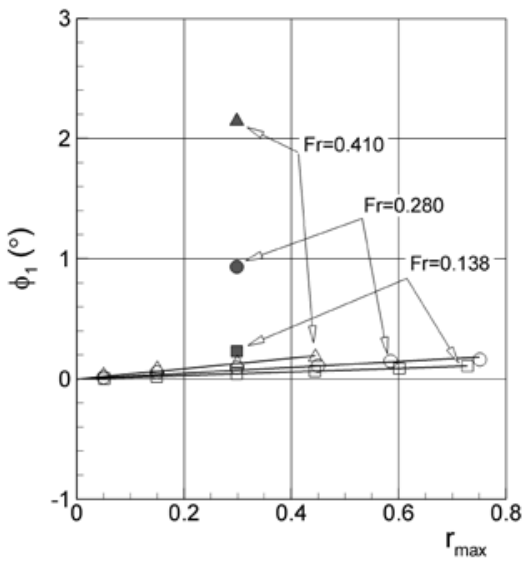

(f)

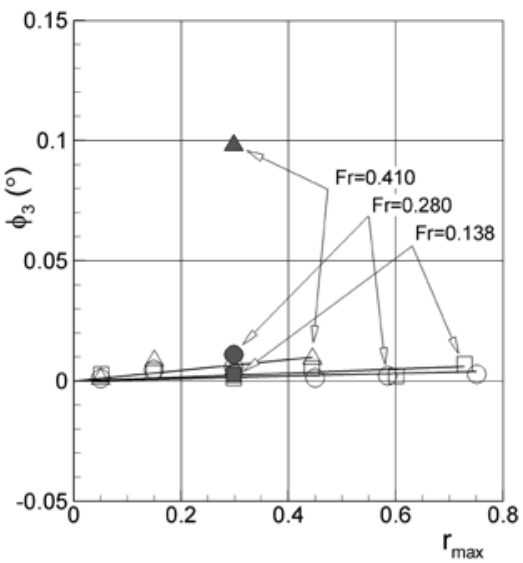

Fig. 19 Harmonic amplitudes of pure yaw heave, pitch, and roll motions: (a) $z_{0}$, (b) $\theta_{0}$, (c) $\phi_{1}$, (d) $z_{2}$, (e) $\theta_{2}$, and (f) $\phi_{3}$. Symbols: $F r=0.138(\Delta), 0.280(\square)$, and $0.410(\mathrm{O})$; Solid-lines with hollow symbols $=\mathrm{FR}_{z \theta}$ and grey-filled symbols $=\mathrm{FR}_{\mathrm{z} \theta \phi}$.

(a)

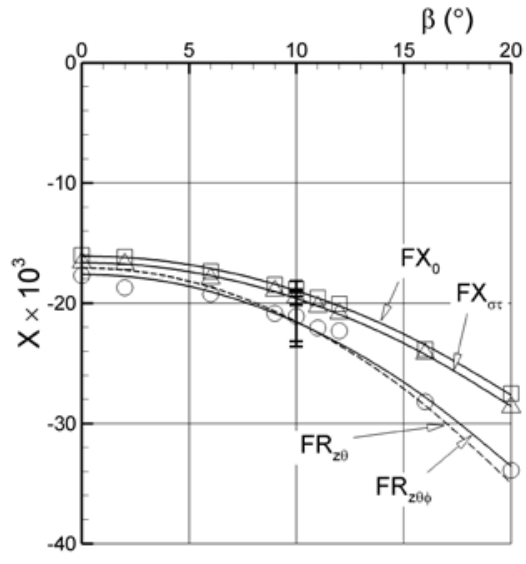

(b)

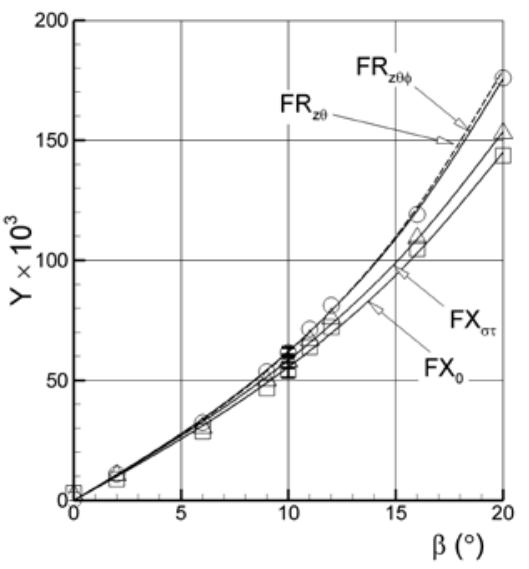

(c)

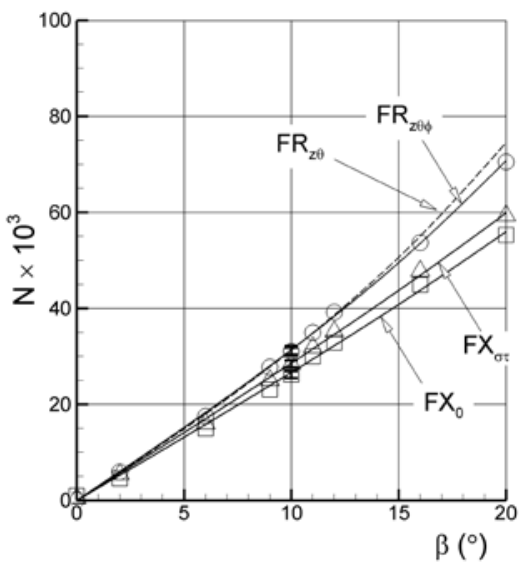

Fig. 20 Comparisons of static drift (a) $X$, (b) $Y$, and (c) $N$ between different mount-conditions $(F r=0.280$ ). Symbols: $\square=\mathrm{FX}_{0}, \Delta=\mathrm{FX}_{\sigma \tau}, \circ=\mathrm{FR}_{\mathrm{z} \theta \phi} ;$ Broken-line $=\mathrm{FR}_{\mathrm{z} \theta}$. 
(a)
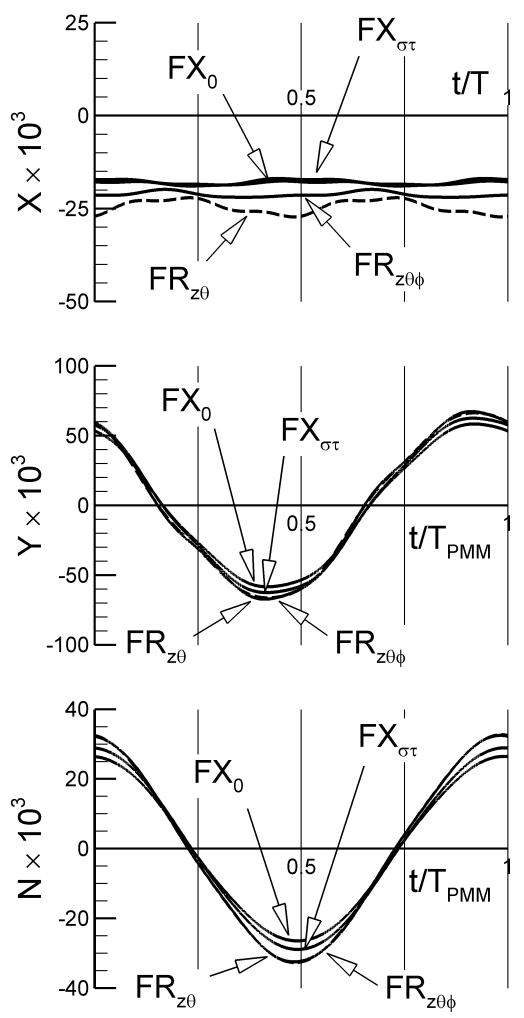

(b)
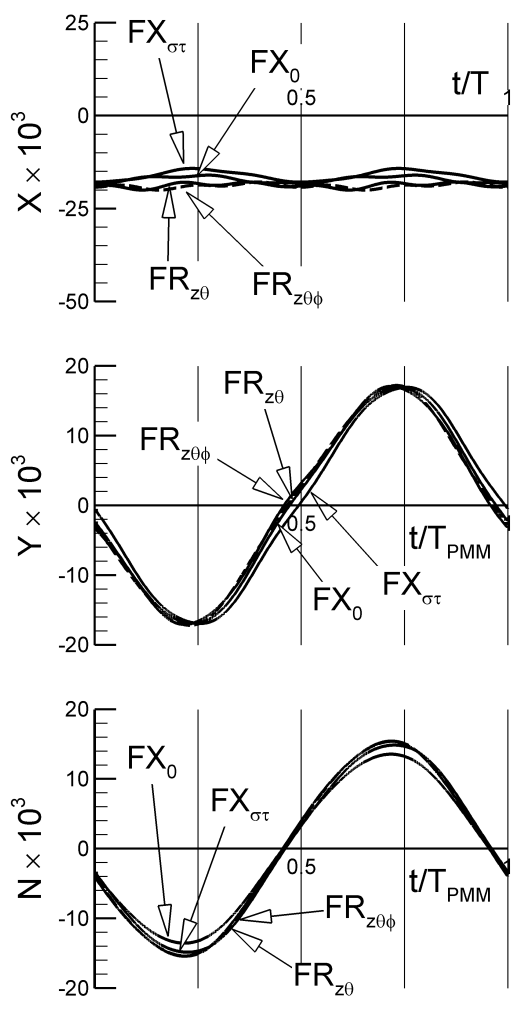
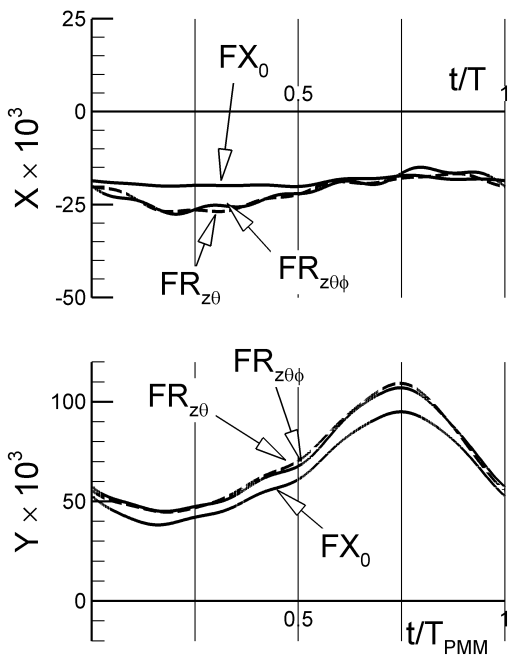

(c)

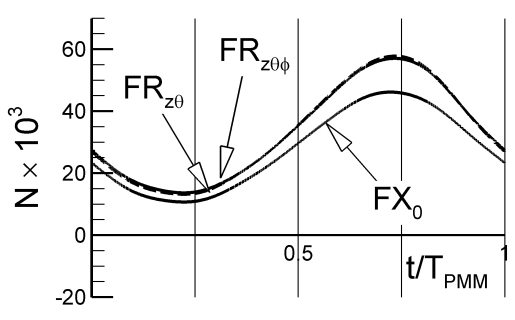

Fig. 21 Comparisons of time-histories of dynamic PMM between different mount-conditions $(F r=0.280)$ : (a) pure sway, $\beta_{\max }=10^{\circ}$, (b) pure yaw, $r_{\max }=0.3$, and (c) yaw-and-drift, $\beta=10^{\circ}$. 